Aus der Klinik für Psychiatrie und Psychotherapie

(Prof. Dr. med. J. Wiltfang)

der Medizinischen Fakultät der Universität Göttingen

\title{
Deskriptive Studie zur zahnärztlichen Betreuung und Versorgung demenzkranker Patienten im Raum Göttingen
}

\author{
INAUGURAL-DISSERTATION \\ zur Erlangung des Doktorgrades \\ für Zahnheilkunde \\ der Medizinischen Fakultät der \\ Georg-August-Universität zu Göttingen
}

vorgelegt von

Anna Sophie Schaper

aus

Bad Hersfeld

Göttingen 2019 
Dekan:

\section{Betreuungsausschuss}

Betreuer/in

Ko-Betreuer/in:

\section{Prüfungskommission}

Referent/in

Ko-Referent/in:

Drittreferent/in:

Datum der mündlichen Prüfung:
Prof. Dr. rer. nat. H. K. Kroemer

Prof. Dr. J. Wiltfang

Prof. Dr. T. Rödig

Prof. Dr. J. Wiltfang

Prof. Dr. H. Bickeböller

Prof. Dr. T. Rödig

31.08 .2020 
Hiermit erkläre ich, die Dissertation mit dem Titel "Deskriptive Studie zur zahnärztlichen Betreuung und Versorgung demenzkranker Patienten im Raum Göttingen" eigenständig angefertigt und keine anderen als die von mir angegebenen Quellen und Hilfsmittel verwendet zu haben. 


\section{Inhaltsverzeichnis}

Abbildungsverzeichnis....................................................................................................................................... III

Tabellenverzeichnis........................................................................................................................................... IV

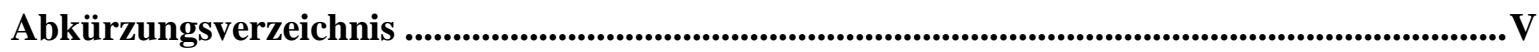

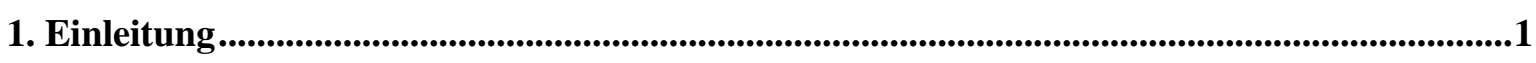

1.1 Gerodontologie: Behandlungsmaßnahmen und -prinzipien.............................................. 1

1.1.1 Gesundheitliche Folgen mangelnder Mundhygiene.................................................... 3

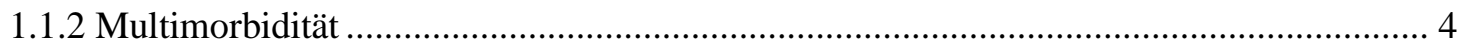

1.1.3 Verbesserung der Behandlung: Rahmenbedingungen und Kommunikation .................... 5

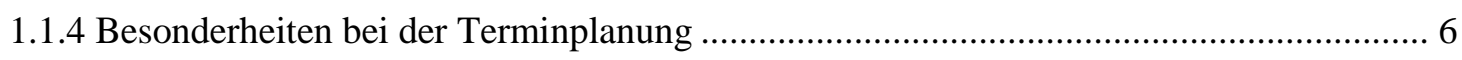

1.1.5 Behandlungsstrategien bei demenziell erkrankten Patienten ........................................... 7

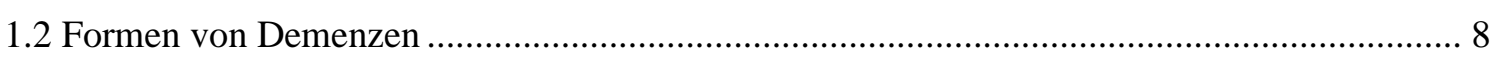

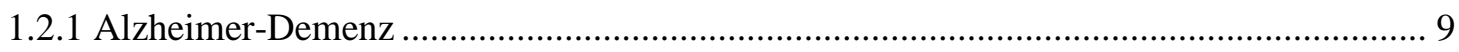

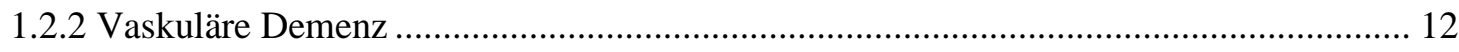

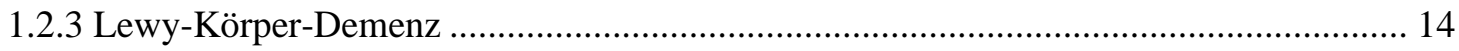

1.3 Problemstellungen in der zahnmedizinischen Behandlung dementer Patienten .................... 15

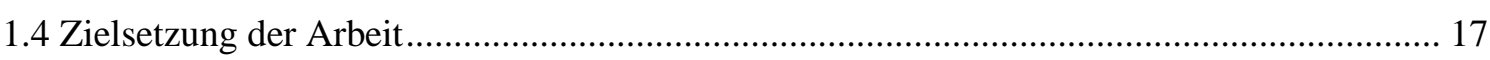

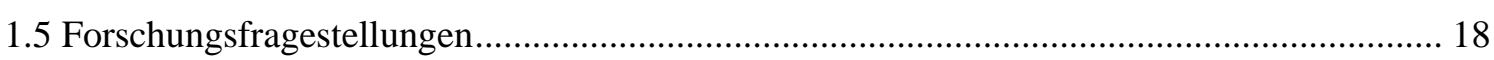

1.5.1 Fragestellung 1: Allgemeine Informationen zur Praxis .............................................. 18

1.5.2 Fragestellung 2: Versorgung und Behandlung demenzkranker Patienten ....................... 20

1.5.3 Fragestellung 3: Beobachtungen bei demenzkranken Patienten in der Praxis ................ 21

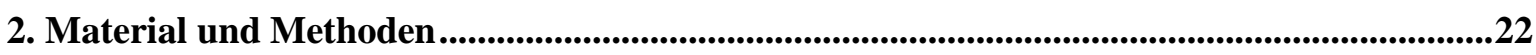

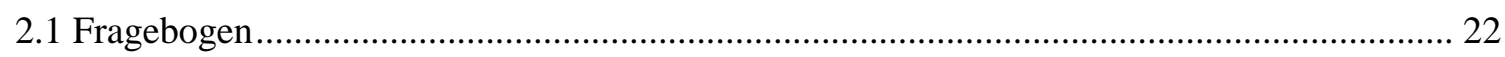

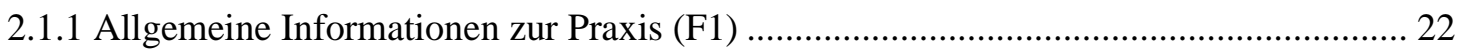

2.1.2 Zahnmedizinische Versorgung demenzkranker Patienten (F2) ...................................... 23

2.1.3 Beobachtungen bei demenzkranken Patienten in der Praxis (F3) ................................. 25

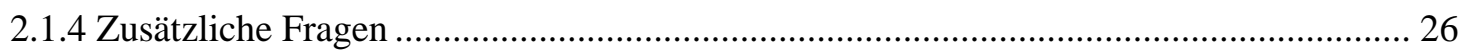

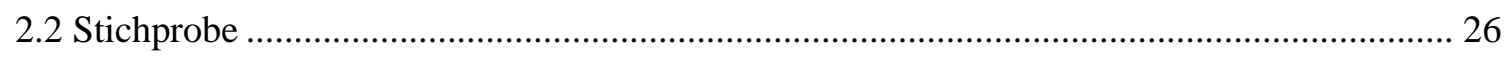

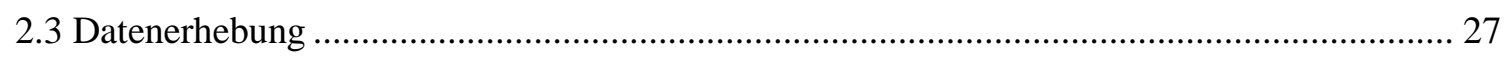

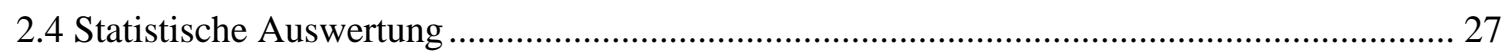




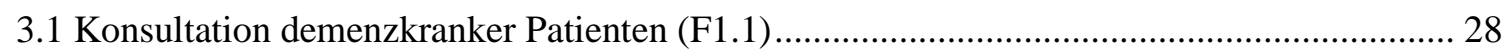

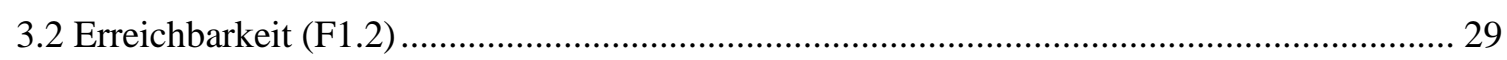

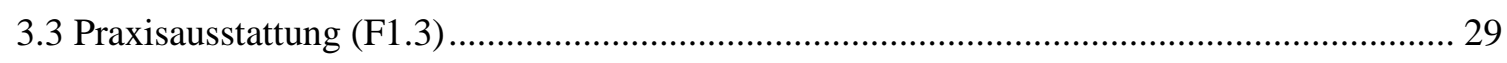

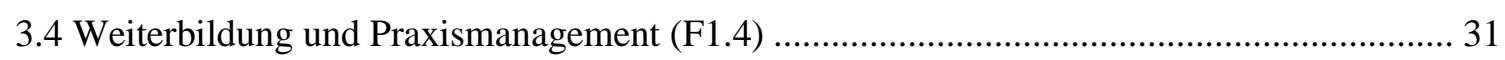

3.5 Besonderheiten in der zahnmedizinischen Versorgung (F2.1) ........................................... 32

3.6 Intraorale Besonderheiten bei demenzkranken Patienten (F2.2) ......................................... 36

3.7 Beobachtung demenzkranker Patienten in der zahnmedizinischen Praxis (F3).................... 38

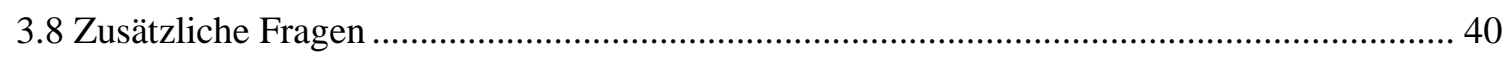

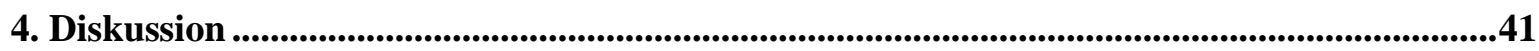

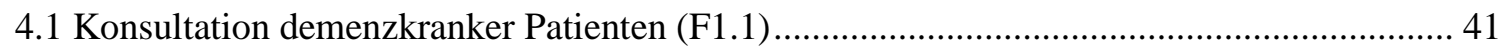

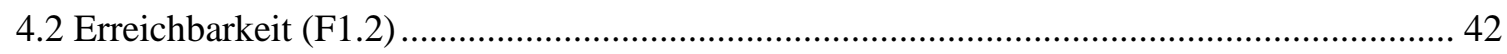

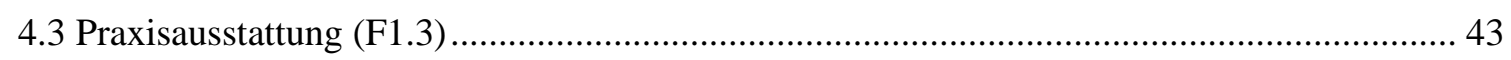

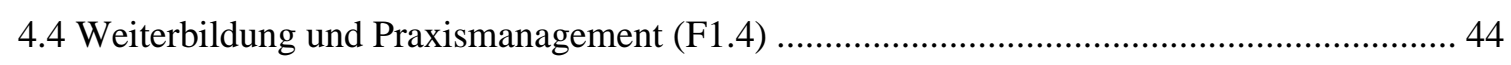

4.5 Besonderheiten in der zahnmedizinischen Versorgung (F2.1) .......................................... 46

4.6 Intraorale Besonderheiten bei demenzkranken Patienten (F2.2) ......................................... 50

4.7 Beobachtung demenzkranker Patienten in der zahnmedizinischen Praxis (F3).................... 52

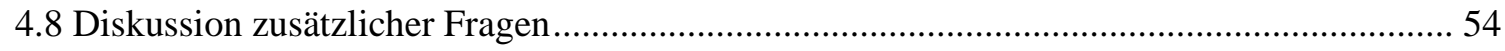

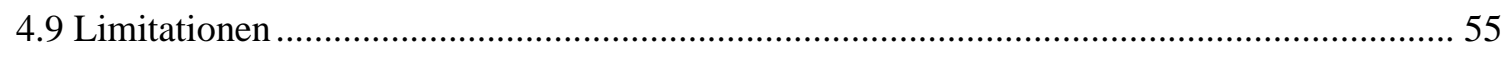

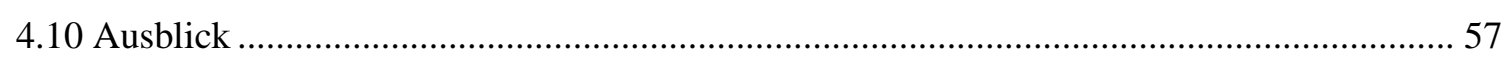

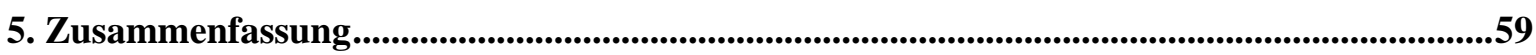

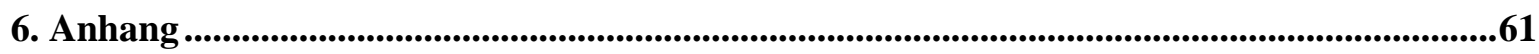

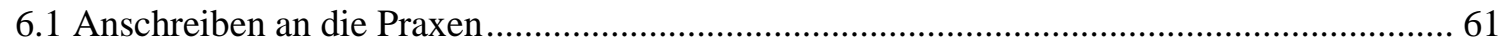

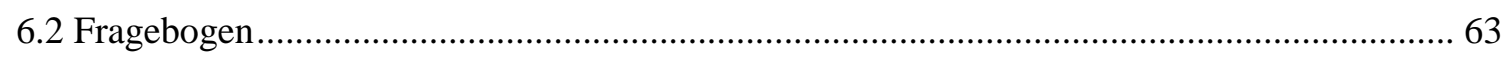

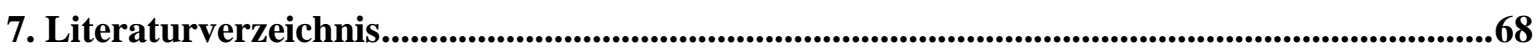




\section{Abbildungsverzeichnis}

Abbildung 1 Kreislauf der Auswirkung einer symptomatischen Therapie mit Psychopharmaka und Sedativa auf die Mundgesundheit 2

Abbildung 2 Kaskade der intraoralen Unterversorgung in Hinsicht auf Unterernährung 3

Abbildung 3 Häufigkeitsverteilung der Demenzformen.

Abbildung 4 Symptomatik und mögliche zahnmedizinische Folgen bei demenzkranken Patienten

Abbildung 5 Prozentuale Auswertung der „Ja vs. Nein“-Antworten zur Konsultation demenzkranker Patienten. 28

Abbildung 6 Prozentuale Auswertung der „Ja vs. Nein“-Antworten zur Erreichbarkeit der Praxis29

Abbildung 7 Prozentuale Auswertung der „Ja vs. Nein“-Antworten zur Praxisausstattung für demenzkranke Patienten

Abbildung 8 Prozentuale Verteilung der behindertengerechten Sanitäreinrichtungen nach Praxisform und der Benutzung von Zahnbänkchen nach Geschlecht

Abbildung 9 Prozentuale Verteilung von „Ja vs. Nein“-Antworten in Hinblick auf weitere Besonderheiten im Praxismanagement und die Bereitschaft zur Weiterbildung.....

Abbildung 10 Prozentuale Verteilung von „Ja vs. Nein“-Antworten in Hinblick auf Besonderheiten in der zahnmedizinischer Versorgung

Abbildung 11 Prozentuale Verteilung empfohlener zahnmedizinischer Pflegeartikel für demenzkranke Patienten getrennt nach Hygienemitteln zur dentalen Pflege, interdentalen Pflege und Prothesenreinigung

Abbildung 12 Prozentuale Angaben zur Putztechnik und Prothesengestaltung nach Praxisform .. 36

Abbildung 13 Intraorale Auffälligkeiten und Erkrankungen bei demenziell erkrankten Patienten37

Abbildung 14 Einschätzung der Häufigkeit von Parodontitis bei durch männliche und weibliche Behandler 38

Abbildung 15 Prozentuale Verteilung der „Ja- vs. Nein"-Antworten in Hinsicht auf die besondere Beobachtung demenzkranker Patienten . 38

Abbildung 16 Zusammenhang zwischen Polypharmazie und intraoralen Erkrankungen 51 


\section{Tabellenverzeichnis}

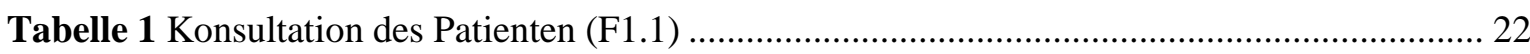

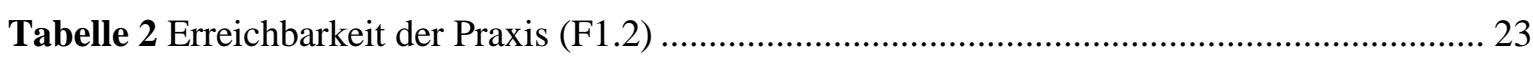

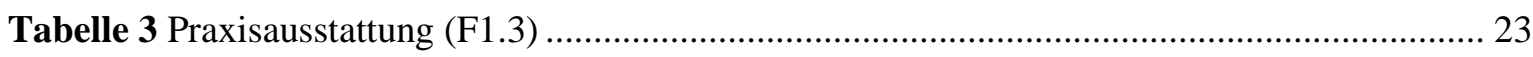

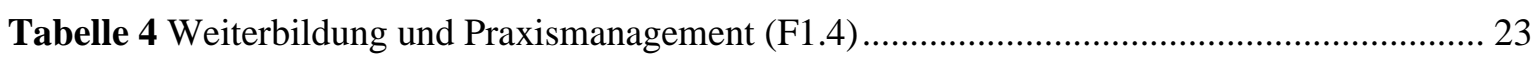

Tabelle 5 Besonderheiten in der zahnmedizinischen Versorgung (F2.1) ..................................... 24

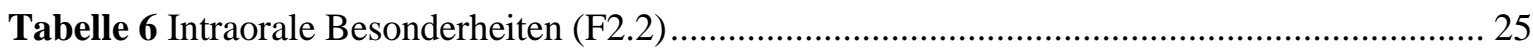

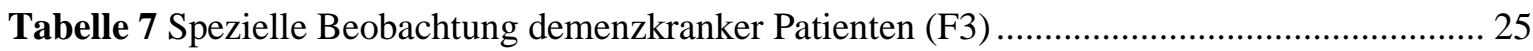

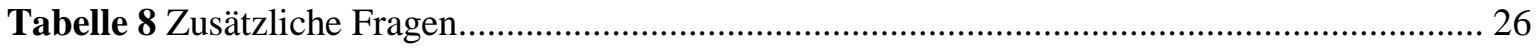




\section{Abkürzungsverzeichnis}

AuB

CT

DemTect

DMS V

ICD

KAI

MMST

MRT

NINCDS/ADRDA

POCD

SÄVIP

\begin{abstract}
Alter und Behinderung
Computertomographie

Demenz-Detektions-Test
\end{abstract}

Fünfte Deutsche Mundgesundheitsstudie

International statistical classification of diseases and related health problems

Kauflächen, Außenflächen, Innenflächen

Mini-Mental-Status-Test

Magnetresonanztomographie

National institute of neurological and communicative disorders and strokel Alzheimer's diseases and related disorders assocication

postoperative cognitive dysfunction

Studie zur ärztlichen Versorgung im Pflegeheim 


\section{Einleitung}

\subsection{Gerodontologie: Behandlungsmaßnahmen und -prinzipien}

Durch den voranschreitenden demographischen Wandel in Deutschland kommt der Gerodontologie eine zunehmende Bedeutung zu. Heute sind bereits $20 \%$ der deutschen Bevölkerung 65 Jahre und älter. Bis zum Jahre 2060 könnte diese Gesellschaftsgruppe auf bis zu 30 \% anwachsen (Zenthöfer et al. 2016). Der alternde Patient stellt in vielerlei Hinsicht eine besondere Herausforderung in der Zahnmedizin dar. Einflussfaktoren wie Polypharmazie, Multimorbidität, die Einschränkung des Bewegungsapparates sowie des Visus und der Hörleistungen müssen während der Behandlung berücksichtigt werden (Bär et al. 2009). Neben den physiologischen Altersveränderungen steigt mit dem zunehmenden Lebensalter auch die Prävalenz für Altersdemenzen: So liegt die Gesamtprävalenz in den westlichen Industrieländern bei den über 65-Jährigen zwischen 5-9 \% (Bickel 2012). Ab dem 65. Lebensalter verdoppelt sich das Risiko, an einer Demenz zu erkranken, etwa alle fünf Jahre. So sind circa ein Drittel der über 90-Jährigen demenziell erkrankt (Mahlberg und Gutzmann 2009). Durch die hirnorganische Störung kommt es sowohl zum Verlust kognitiver Funktionen wie auch Alltagskompetenzen und somit zum Eintritt in die Pflegebedürftigkeit. Häufig bleiben dadurch viele Risiken der Mundhöhle, wie vernachlässigte Pflege der Restbezahnung und des Zahnersatzes sowie dentale Pathologien, unbehandelt und unerkannt. In der Fünften Deutschen Mundgesundheitsstudie (DMS V) wird berichtet, dass eine höhere Karieserfahrung bei älteren Menschen mit Pflegebedarf vorliegt als in der gesamten Altersgruppe der älteren Senioren (75-100 Jahre). Zusätzlich sind bei dieser Patientengruppe weniger eigene Zähne sowie häufiger ein herausnehmbarer Zahnersatz zu verzeichen. Gleichzeitig sinkt jedoch die Zahnlosigkeit in der Gruppe der Senioren, sodass im Durchschnitt fünf eigene Zähne vorhanden sind, mehr als im Jahr 1997. Knapp ein Drittel der pflegebedürftigen Menschen sind nicht mehr eigenständig in der Lage den Restzahnbestand adäquat zu reinigen und zu pflegen. Auch nötige Zahnarzttermine können von $60 \%$ der Pflegebedürftigen nicht mehr organisiert werden (Jordan und Micheelis 2016). Der Bedarf nach zahnmedizinischer Versorgung steigt mit zunehmendem Alter an, der Zahnarztkontakt jedoch nicht. Laut der Studie zur ärztlichen Versorgung im Pflegeheim (SÄVIP-Studie) hatten $10 \%$ der Bewohner in den befragten Pflegeeinrichtungen im Jahr vor der Befragung keinen Zahnarztkontakt, $36 \%$ hatten ein bis drei Zahnarztkontakte im Jahr (Hallauer et al. 2005). Laut Knabe und Kram (1997) waren nur 34,1 \% der als pflegebedürftig eingestuften älteren Menschen in den letzten fünf Jahren in zahnmedizinischer Behandlung. Diese Befunde können einerseits dadurch erklärt werden, dass der Zugang zu zahnärztlicher Versorgung in Pflegeheimen eingeschränkt ist und zum anderen, dass die Zahnpflege in hohem Maße dem Pflegepersonal obliegt, bei welchem teilweise Wissensdefizite in Bezug auf die individuellen Anforderungen bei der Mundpflege von Senioren, insbesondere beim Umgang mit demenziell erkrankten Patienten, vorliegen und zudem der zeitliche Rahmen begrenzt ist (Zenthöfer et al. 2016). Um eine Verbesserung dieser Situation in Pflegeheimen 
zu erzielen, fordern Besimo und Besimo-Meyer (2015) in ihrer Studie zur Aufzeigung der interdisziplinären Anforderungen für eine verbesserte orale Prävention und Therapie: (1) Eine interdisziplinäre Feststellung zwischen Hausarzt, Pflegepersonal und Zahnarzt als Ausgangspunkt, (2) der Behandler ist hinsichtlich der Zielgruppe geschult und ausgebildet - somit können die besonderen Bedürfnisse der gerontopsychiatrisch erkrankten Patienten berücksichtigt werden.

Das Resultat, welches sich aus der Unterversorgung von pflegebedürftigen Senioren ergibt, ist eine eingeschränkte Mundhygiene mit teilweise desolaten Gebisszuständen, welche auf einer mangelnden Pflege und Reinigung sowie Unwissenheit des Pflegepersonals oder der Angehörigen beruht. Eine weitere Problematik stellt in diesem Zusammenhang die Schmerzdetektion bei kommunikativ und kognitiv eingeschränkten Senioren dar. Viele pflegebedürftige Personen sind nicht mehr in der Lage, Auskunft über Schmerzzustände zu geben und diese zu lokalisieren. Diese Missempfindungen äußern sich meist in Form von unruhigem oder aggressivem Verhalten, Lautäußerungen oder Abwehrverhalten. Daraus resultieren Verhaltensauffälligkeiten, welche häufig symptomatisch mit $u$. a. Psychopharmaka oder Seditiva behandelt werden. Die eigentliche Behebung der intraoralen Ursache bleibt in diesen Fällen meist aus (Bornemann-Cimenti et al. 2012) (s. Abbildung 1).

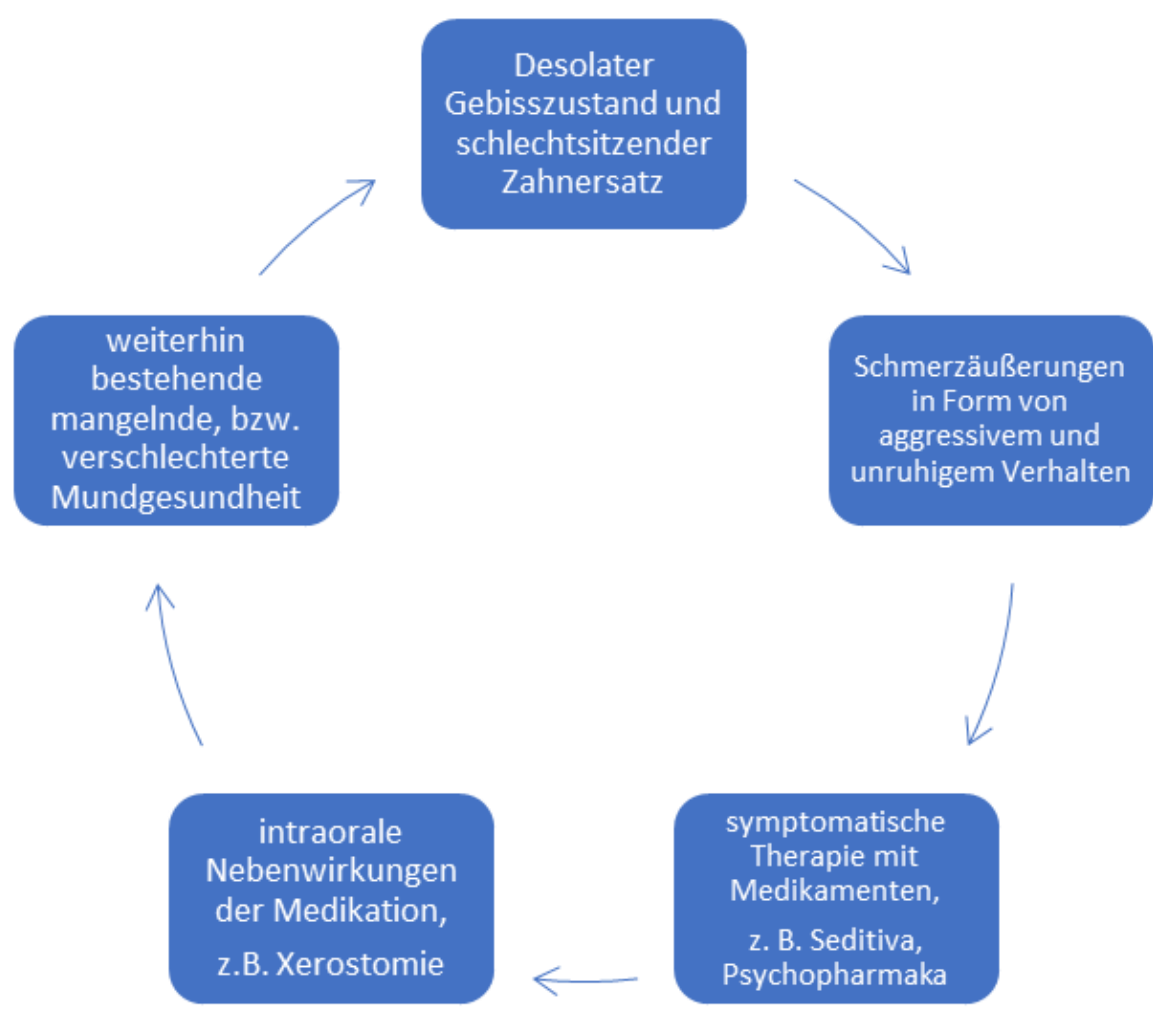

Abbildung 1 Kreislauf der Auswirkung einer symptomatischen Therapie mit Psychopharmaka und Sedativa auf die Mundgesundheit (basierend auf Bornemann-Cimenti et al. 2012) 


\subsubsection{Gesundheitliche Folgen mangelnder Mundhygiene}

Die weiterhin bestehende mangelnde Mundgesundheit und die persistierenden oralen Pathogene haben direkte oder indirekte Auswirkungen auf den allgemeinen Gesundheitszustand. In den Körperkreislauf eindringende Pathogene können in artherosklerotischen Plaques nachgewiesen werden und zu einer erhöhten Prävalenz für kardiovaskuläre Erkrankungen und Schlaganfälle führen (Zenthöfer et al. 2016). Auch ein wechselseitiger Zusammenhang zwischen Diabetes und Parodontalerkrankungen konnte nachgewiesen werden (Zenthöfer et al. 2016). Bei bettlägerigen Pflegefällen steigt zudem durch die Aspiration von bakteriellen Pathogenen das Risiko für Pneumonien, welche eine häufige Todesursache in der Geriatrie darstellen (SGZBB 2015). Des Weiteren nimmt die intraorale Unterversorgung einen substanziellen Einfluss auf den Ernährungszustand. Unterernährung stellt mit $20 \%$ ein relevantes geriatrisches Problem dar, welches durch das absolute Lebensalter, Pflegebedürftigkeit und Demenzerkrankung begünstigt wird (Zenthöfer et al. 2016). Zudem kann eine Verminderung der Kaukraft und Kaueffizienz zu Malnutrition, Hyposalivation, Schwächung des Immunsystems und stark vermindertem Wohlbefinden führen (SGZBB 2015). Symptome einer Mangelernährung können im Mund- und Gesichtsbereich sichtbar werden, z. B. in Form von verzögerter Wundheilung, einer gestörten Immunabwehr, einer Anämie mit blasser Mundschleimhaut, glatte Rötungen der Mundschleimhaut oder peripherer Neuropathien (Glenz et al. 2015) (s. Abbildung 2).

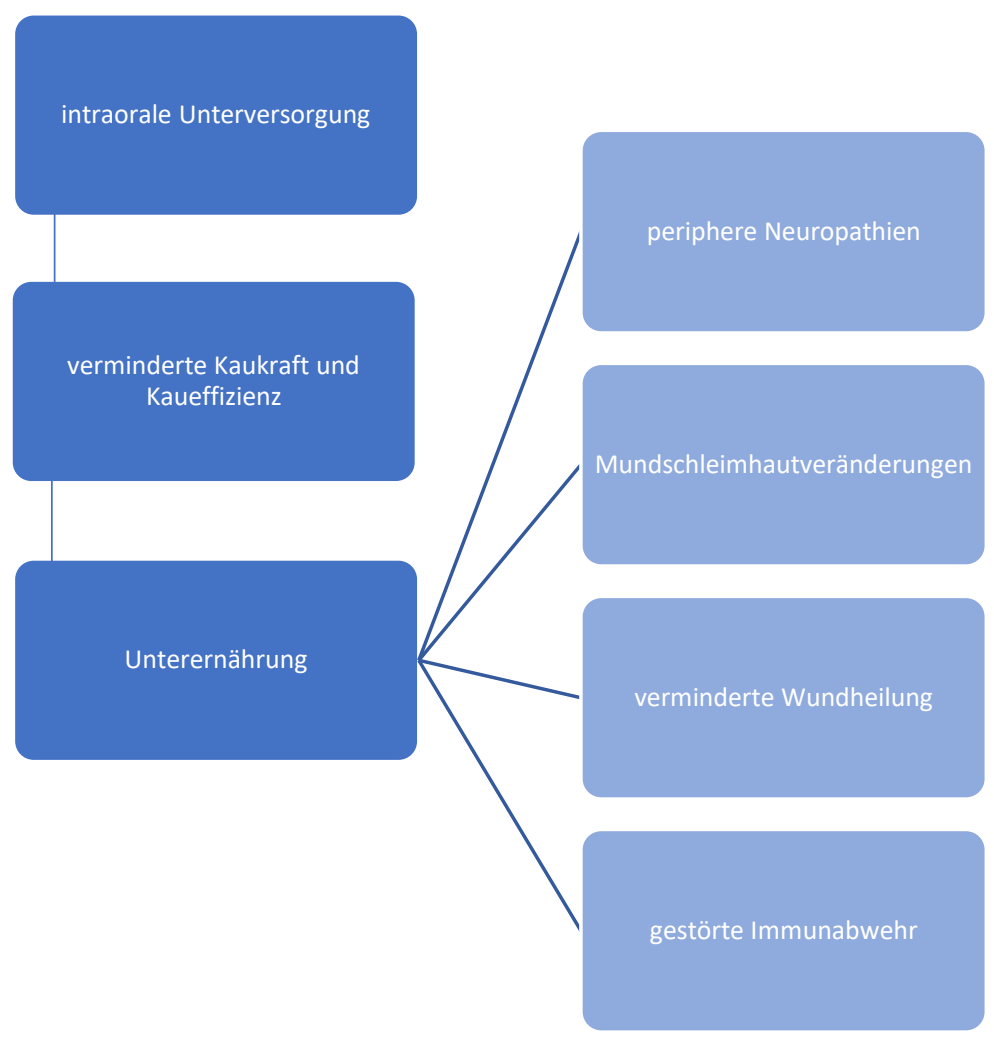

Abbildung 2 Kaskade der intraoralen Unterversorgung in Hinsicht auf Unterernährung (basierend auf Glenz et al. 2015) 


\subsubsection{Multimorbidität}

Ein weiteres Charakteristikum des geriatrischen Patienten ist die Multimorbidität, da der Alterungsprozess die Inzidenz chronischer Krankheiten begünstigt (Zenthöfer et al. 2016). Einige Autoren definieren die Multimorbidität durch das gleichzeitige Auftreten von bereits zwei Erkrankungen, in anderen Fällen wird das Zusammentreffen von fünf oder mehr Erkrankungen als Multimorbidität bezeichnet. Kuhlmey und Tesch-Römer (2013) beschreiben darüber hinaus die geriatrietypische Multimorbidität, die weniger durch das Zusammentreffen von Krankheiten, sondern durch die Anwesenheit multimorbiditätsassoziierter Phänomene, wie beispielsweise Schmerz, Immobilität, Sensibilitäts- und sensorische Störungen, sowie durch psychische Störungen gekennzeichnet ist. Diese Erkrankungen und Symptome werden häufig polypharmazeutisch therapiert, was einen substantiellen Einfluss auf die Mundgesundheit haben kann und in jeder zahnmedizinischen Behandlung gesondert berücksichtigt werden muss (Zenthöfer et al. 2016). Im Durchschnitt erhalten Menschen über 70 Jahre drei Arzneimittel pro Tag. Jeder Dritte Patient dieser Altersgruppe bekommt fünf bis acht verschiedene Medikamente, jeder Sechste sogar mehr als 13 (Bär et al. 2009). Die in der Gerontopsychiatrie häufig zum Einsatz kommenden Medikamentengruppen der Antidepressiva, Antipsychotika oder Sedativa können als Nebenwirkung zu Mundtrockenheit (Xerostomie) führen, welche die Plaqueanlagerung begünstigt und damit das Risiko für Karies, Zahnfleischerkrankungen und Infektionen durch orale Pathogene, erhöht. Antipsychotika können zudem zu unfreiwilligen Zungen- und Kieferbewegungen führen, welche das Tragen einer Prothese erschweren und somit das Risiko der Unterernährung und Malnutrition erhöhen (Mathers 2015). Auch andere Medikamentengruppen, die bei geriatrischen Patienten häufig zum Einsatz kommen, wie der Kalziumantagonist Nifedipin oder das Immunsupressivum Ciclosporin können sich negativ auf Parodontalerkrankungen auswirken oder sogar hervorrufen (Zenthöfer et al. 2016). Auch hinsichtlich der zahnmedizinischen Behandlung nimmt die Multimedikation, laut Zenthöfer et al. (2016) einen relevanten Einfluss. Bei der Dosierung zahnärztlichen Medikamente, wie Anästhetika, Antibiotika und Analgetika muss auf die reduzierte Muskelmasse bei Senioren (Sarkopenie), sowie Funktionsveränderungen von Leber und Niere Rücksicht genommen werden. Auch müssen Wechselwirkungen mit regelmäßig eingenommenen Medikamenten beachtet werden, um unerwünschte Arzneimittelnebenwirkungen zu umgehen, wie es bei Patienten mit 14 Medikamenten zu rund $20 \%$ der Fall ist (Zenthöfer et al. 2016).

Die geriatrietypische Multimorbidität fordert während der zahnmedizinischen Behandlung gesonderte Strategien und Maßnahmen. Bereits der Weg in die Praxis bereitet dem immobilen und instabilen Patienten einen hohen psychischen und physischen Aufwand. Eine gut ausgeschilderte Praxis mit Busanbindung, barrierearmem Zugang und Innenbereich erleichtert Senioren den ersten Schritt zum Zahnarztstuhl. Laut Nitschke et al. (2015) zeigt sich, dass patientenspezifische Faktoren, wie Gebrechlichkeit, Immobilität, psychisch und kognitive Einschränkungen die Inanspruchnahme 
zahnärztlicher Leistungen negativ beeinflussen und zu einer Verschlechterung der intraoralen Situation führen. Bettlägerige und nicht transportfähige Patienten sind auf Haus- bzw. Heimbesuche seitens des Zahnarztes angewiesen. Für diese Fälle werden Online-Materialchecklisten von den Landeszahnärztekammern bereitgestellt und auf dem Markt mobile Behandlungseinheiten angeboten. Ludwig (2016) fordert beim Einsatz jener Einheiten jedoch eine kritische Hinterfragung, weil diese dazu verleiten aufwendigere Behandlungen mit entsprechenden Risiken durchzuführen. Hausbesuche seien in erster Linie dazu geeignet sich ein Bild von der Situation zu machen und nur risikofreie Behandlungen fachgerecht durchzuführen. Für invasive Behandlungen sollte der Transport in eine geeignete Einrichtung erfolgen (Ludwig 2016). Die zahnmedizinische Behandlung von pflegebedürftigen Patienten wird zum einen durch die hohen Transportkosten in Praxen oder Kliniken erschwert, zum anderen durch das übliche Honorar der gesetzlichen Krankenkassen, wodurch eine generelle mobile Betreuung betriebswirtschaftlich nicht darstellbar ist (DettbarnReggentin und Reggentin 2016). Bereits 2010 wurde von der Kassenzahnärztlichen Bundesvereinigung, der Bundeszahnärztekammer und wissenschaftlichen Organisationen ein Konzept für Alter und Behinderung (AuB-Konzept) entwickelt, welches die Einbeziehung Pflegebedürftiger und Menschen mit Behinderung in die vertragszahnärztliche Versorgung ermöglichen soll (Schmitt 2017). Die Vertragszahnärzte haben demnach Anspruch auf ein höheres Honorar, bei der Durchführung von Hausbesuchen und Besuchen in Pflegeheimen. 2013 wurden mit dem Versorgungsstrukturgesetz und dem Pflege-Neuausrichtungsgesetz bis dahin geltende Besuchspositionen neu definiert, indem im Bewertungsmaßstab für zahnärztliche Leistungen neue Zuschlagpositionen für personellen, instrumentellen und zeitlichen Mehraufwand, aufgenommen wurden (Schmitt 2017). Ein weiterer Schritt zur Verbesserung der zahnmedizinischen Situationen Pflegebedürftiger soll im Juli 2018 vorgenommen werden, indem eine gesetzliche Verankerung zur Inanspruchnahme präventiver, unter anderem privater zahnmedizinischer Leistungen der Krankenkasse, erfolgen soll. Künftig können zur Verhütung von Zahnerkrankungen bei Pflegebedürftigen entsprechende Leistungen (z. B. Entfernung harter Zahnbeläge, Erhebung eines Mundgesundheitsstatus mit Erstellung eines Behandlungsplans weitere Aufklärungsmaßnahmen) in Anspruch genommen werden. Verbesserungen zeigen sich in Form von steigenden Kooperationsverträgen zwischen Zahnärzten und Pflegeheimen, welche bundesweit einen Abdeckungsgrad von $24 \%$ im Jahr 2016 betrugen (Schmitt 2017).

\subsubsection{Verbesserung der Behandlung: Rahmenbedingungen und Kommunikation}

Um die Kooperation und die Kommunikation während der Behandlung zu verbessern, können Hilfsmittel, wie konfektionierte Lesebrillen und Hörgeräte, Zahnbänkchen zum Offenhalten des Mundes, Kissen zur Verbesserung der Lagerung und Kopfstützen für Rollstühle, zum Einsatz kommen (Nitschke et al. 2015). Zur Veranschaulichung und Vereinfachung werden an die Belange 
der Patientengruppe angepasste Kommunikationsmittel, Flyer und Broschüren in Kombination mit einer kognitiv an Senioren angepassten Gesprächsführung, empfohlen (Bär et al. 2009). So sollte die Kommunikation auf Augenhöhe und unter Blickkontakt stattfinden. Des Weiteren ist das Formulieren einfacher, kurzer Sätze empfehlenswert, die bei Missverständnis wiederholt werden können. Einfache, direkte Fragen vereinfachen die Verständlichkeit und lassen weniger Raum für Interpretationen. Für demente Patienten empfiehlt es sich, dass diese immer im selben Behandlungsraum und vom selben Behandlungsteam betreut werden, um Desorientiertheit vorzubeugen und eine vertraute Atmosphäre zu schaffen (Wernke und Steinbock 2015). Auch Familienangehörige oder Pflegepersonal sollten in die Behandlung und Planung einbezogen und hinsichtlich der Prävention und Pflege eingebunden werden. Des Weiteren muss bei dementen, nicht einwilligungsfähigen Patienten aus juristischen Gründen der gesetzliche Betreuer (meist Familienangehörige) für die Gesundheits- und Vermögenssorge anwesend sein, da der Patient durch seine kognitive Einschränkung nicht mehr in der Lage ist, eigenständige Entscheidungen zu treffen (Ludwig 2016).

\subsubsection{Besonderheiten bei der Terminplanung}

Um eine adäquate an dem Patienten angepasste Recall-Struktur $\mathrm{zu}$ gewährleisten, müssen allgemeinmedizinische und organisatorische Besonderheiten bei der Terminplanung mit in Betracht gezogen werden. Neben Pflegeprozessen, administrativen Abläufen und Bringdiensten in den Pflegeinrichtungen oder der Häuslichkeit, muss Rücksicht auf Medikamenteneinnahmezeiten oder Essenszeiten, vor allem bei Diabetikern, genommen werden. Die Terminvergabe sollte dabei sensibel erfolgen und an die Bedürfnisse des Patienten angepasst sein (Nitschke et al. 2015). Besonders bei dementen Patienten sollte nach Diagnosestellung ein Zahnarzt aufgesucht werden um die vorhandene Kooperation im Anfangsstadium auszunutzen und den Patienten auf einen möglichst hohen Standard zu sanieren. Anschließend steht eine Erhaltungstherapie in den weiteren Krankheitsstadien im Vordergrund. Erfolgt nach Diagnosestellung keine Sanierung des Gebisszustandes und wird diese in einem späteren Stadium von Nöten, sind oft nur noch radikale Lösungen im Rahmen einer Vollnarkose möglich (Bär et al. 2009). Bei der Behandlung geriatrischer Patienten unter Vollnarkose muss jedoch eine strenge Abwägung zwischen Risiken und Nutzen seitens des Zahnarztes gestellt werden. Als mögliche Komplikation muss in diesem Zusammenhang bedacht werden, dass es zu einer Postoperative cognitive dysfunction (POCD) kommen kann, welche in unmittelbaren Zusammenhang mit dem Alter der Patienten steht und zu einer Verschlechterung des neuropsychiatrischen Zustandes führt (Kratz et al. 2005) 


\subsubsection{Behandlungsstrategien bei demenziell erkrankten Patienten}

Hinsichtlich der zahnmedizinischen Versorgung muss bei dementen und betagten Patienten ein gesondertes Behandlungskonzept angestrebt werden. Im Rahmen der Therapieentscheidung müssen Einflussfaktoren wie Multimorbidität, verminderte Motorik, Sensorik und kognitive Fähigkeit mit einbezogen werden. Häufig liegt bei dieser Patientengruppe eine verminderte Adaptionsfähigkeit vor, welche die Umstellung von festsitzenden auf herausnehmbaren Zahnersatz erschweren kann. Der Zahnarzt sollte diesbezüglich in Betracht ziehen, dass eine frühere Umstellung auf nicht festsitzenden Zahnersatz mit entsprechenden Erweiterungsmöglichkeiten eine langfristigere Lösung zur Erhaltung der Kautätigkeit, Phonation und intraoraler Gesundheit darstellen könnte. Ein Kompromiss seitens des Zahnarztes, einen Zahnersatz geringeren Standards anzufertigen, ist meist unumgehbar (Bär et al. 2009). Wöstmann (2003) fordert einen leicht handhabbaren, pflegefähigen und robusten Zahnersatz für betagte Patienten um das Kauorgan möglichst lange funktionsfähig zu halten. Bewährt haben sich für diese Sicherstellung vor allem Doppelkronen, da sie im Gegensatz zu Stegen oder Geschieben einfach zu reinigen sind, einen guten Sitz gewährleisten und einfach eingegliedert werden können (Wöstmann 2003). Bär et al. (2009) empfiehlt besonders bei dementen Patienten und Patienten in Pflegeeinrichtungen, deren Namen in die Prothese einzuarbeiten und ein Duplikat anfertigen zu lassen, falls es zu einem Verlust des Zahnersatzes kommt. Durch Duplikate wird eine Neuanfertigung vereinfacht und eine zusätzliche Behandlung bei unkooperativen Patienten kann umgangen werden, wodurch auch deren Belastung reduziert wird (Bär et al. 2009).

Um eine eventuelle frühzeitige kognitive Einschränkung des Patienten zu identifizieren, ist es hilfreich Screeningverfahren anzuwenden. Im zahnmedizinischen Alltag hat sich durch seine einfache und schnelle Durchführung der Uhrentest besonders bewährt (Glenz et al. 2015). Er dient insbesondere der Beurteilung der exekutiven Kognition und gibt Hinweis auf eine erste Einschätzung der Adaptionsfähigkeit des Patienten. Dieser wird dazu angehalten eine Uhr mit vorgegebener Uhrzeit aufzuzeichnen. Geschultes Personal kann das Ergebnis anschließend beurteilen und eine vorläufige Diagnose vermerken. Bei pathologischem Ergebnis muss eine Überweisung zum Facharzt erfolgen um eine weitere Abklärung zu veranlassen. Ein weiteres Instrument zur Beurteilung kognitiver Einbußen ist der Mini-Mental-Status-Test (MMST). Dieser Test dient der Beurteilung des Schweregrades einer Demenz und ist als Ergänzung der neuropsychologischen Testung gedacht. Dabei handelt es sich um eine Befragung mit Handlungsaufgaben aus den Bereichen Merkfähigkeit, Erinnerungsfähigkeit, Orientierung, Aufmerksamkeit, Rechenfähigkeit und Sprache. Es kann eine maximale Punktzahl von 30 erreicht werden. Liegt das Testergebnis unter 20 Punkten wird eine Demenz angenommen. Unter zehn Punkten liegt eine schwere Demenz vor (Bühler 2014). Der aus fünf Untereinheiten bestehende Demenz-Detektions-Test (DemTect) unterscheidet anhand des Alters in „altersgemäße Leistung“, „leichter kognitiver Einschränkung“ und „Demenz-Verdacht“. Dieser Test ist in zehn Minuten durchführbar und umschließt Aufgaben wie beispielsweise zehn 
vorgelesene Wörter zu wiederholen, Zahnlenfolgen in umgedrehter Reihenfolge wiederzugeben, sowie Zahlen sowohl als Zahlenwort als auch umgekehrt zu schreiben. Die Auswertung erfolgt in Hinblick auf das Alter (jünger und älter als 60 Jahre) mit Hilfe einer Umrechnungstabelle (Kessler et al. 2000).

Um der vulnerablen Gruppe der geriatrischen und dementen Patienten eine adäquate, individuell angepasst zahnmedizinische Behandlung und Versorgung $\mathrm{zu}$ gewährleisten sind gesonderte Behandlungsmaßnahmen und -prinzipen angezeigt. Behandler und Personal müssen hinsichtlich der besonderen Bedürfnisse dieser Patientengruppe geschult und ausgebildet sein. Der kontinuierlich fortschreitende Prozess des Alterns steht im Mittelpunkt der Seniorenzahnmedizin und bedarf durch Berücksichtigung der Lebenssituation und des Allgemeinzustandes einer gesonderten Nachsicht im Rahmen der zahnmedizinischen Behandlung und der Anpassung des individuell optimierten Zahnersatzes.

Im Folgeneden soll ein Überblick über die Demenzformen gegeben werden, die bei den zu behandelnden Patienten mit der höchsten Wahrscheinlichkeit auftreten. Weiterhin soll auf die durch demenzielle Symptome bedingte Problematik eingegangen werden, welche in der zahnmedizinischen Behandlung von Patienten mit der jeweiligen Demenzform auftreten kann.

\subsection{Formen von Demenzen}

Demenzen definieren sich nach der International statistical classification of diseases and related health problems (ICD) zum einen über kognitive Störungen im Bereich des Denkvermögens, wie der Beeinträchtigung der Informationsverarbeitung und der Verminderung des Ideenflusses, sowie zum anderen über Gedächtnisstörungen, wie der Wiedergabe und Aufnahme neuer Informationen oder dem Schwund früher erlernter und vertrauter Inhalte. Begleitet werden diese Einschränkungen im kognitiven Bereich bei fast allen Patienten durch neuropsychiatrische Störungen, wie beispielsweise Veränderungen des Sozialverhaltens, der Impulskontrolle und der Motivation. Diese Störungen müssen mindestens sechs Monate vorliegen und eine wesentliche Beeinträchtigung der Alltagsaktivitäten nach sich ziehen (Mahlberg und Gutzmann 2009).

Medizinisch unterscheidet man primäre und sekundäre Demenzen. Bei den neurodegenerativen oder primären Formen, liegt eine Schädigung von Nervenzellen vor, die durch die Fehlfaltungen von Proteinen getriggert ist und zu einer lokalen Ausbreitung des pathologischen Prozesses führen kann. Sekundäre Demenzformen entstehen im Gegensatz dazu durch „äußere“ Einflüsse, wie beispielsweise vaskuläre Läsionen oder metabolischen Erkrankungen, bei denen Nervenzelle zu Schaden kommen (Gasser und Maetzler 2012). 
Die Gesamtprävalenz der Demenz liegt in den westlichen Industrieländern bei den über 65-Jährigen zwischen 5-9 \% (Bickel 2012). Ab dem 65. Lebensalter verdoppelt sich das Risiko an einer Demenz zu erkranken um etwa alle fünf Jahre. So sind etwa ein Drittel der über 90-Jährigen demenziell erkrankt (Mahlberg und Gutzmann 2009). Aufgrund der höheren Lebenserwartung sind Frauen in der Risikogruppe der Hoch- und Höchstbetagten häufiger vertreten als Männer und somit häufiger von einer Demenzerkrankung betroffen (Bickel 2012). In Abbildung 3 ist die Häufigkeitsverteilung von Demenzerkrankungen dargestellt. Mit $60 \%$ entfällt der Großteil demenzieller Erkrankungen auf die Alzheimer-Demenz. Die zweithäufigste Form mit $15 \%$ ist die vaskuläre Demenz. An dritter Stelle finden sich mit einer Prävalenz von $15 \%$ Demenzen mit Lewy-Körperchen. Die übrigen Demenzenformen machen nur weniger als $10 \%$ aus. Uneinheitliche Diagnosekriterien und eine hohe Anzahl an Mischformen führen jedoch zu Schwankungen der Häufigkeitsangaben (Mahlberg und Gutzmann 2009). Im weiteren Verlauf wird auf die drei häufigsten Formen der Demenz näher eingegangen. ${ }^{1}$

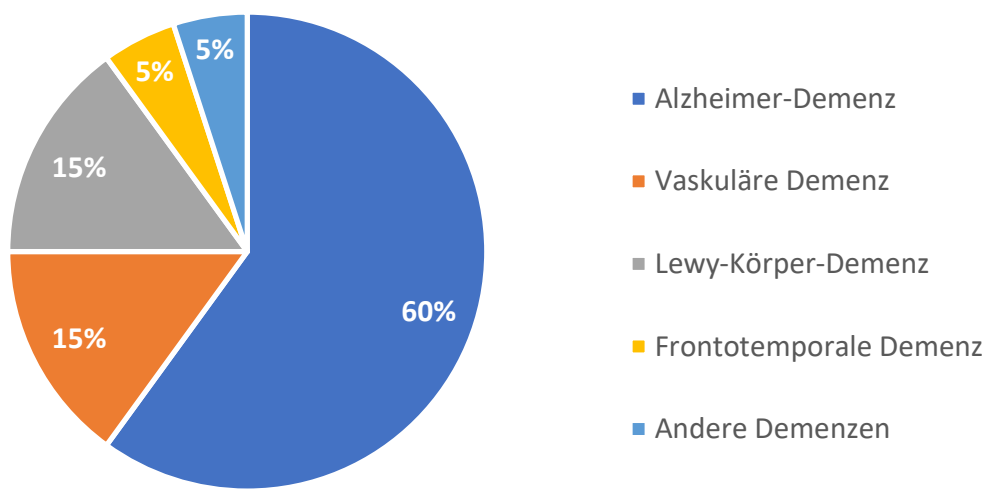

Abbildung 3 Häufigkeitsverteilung der Demenzformen (basierend auf Mahlberg und Gutzmann 2009)

\subsubsection{Alzheimer-Demenz}

Die häufigste primäre Demenzform stellt die Alzheimer-Demenz dar und wurde bereits 1907 von dem deutschen Psychiater Alois Alzheimer beschrieben. Sie gilt als Paradigma der altersabhängigen demenziellen Erkrankung, bei der höhere kognitive Funktionen irreversibeln, progressiven Störungen unterliegen (Rapp 2009). Bei den Betroffenen ist eine diffuse Hirnatrophie erkennbar, die histologisch von Neurofibrillenveränderungen, senilen Plaques und Dentridenschwunden sowie einer Verringerung synaptischer Verbindungen gekennzeichnet ist und schon Jahre vor den ersten

\footnotetext{
${ }^{1}$ Aufgrund der Diagnosevergabe nach ICD-10 soll in der vorliegenden Arbeit nicht näher auf die Definition demenzieller Erkrankungen nach DSM-IV bzw. DSM-V eingegangen werden. Siehe hierzu eine vergleichende Darstellung von Maier und Barnikol 2014.
} 
klinischen Symptomen auftritt (Tölle und Windgassen 2014). Auf Neurotransmitterbene kommt es zu einer Reduktion nikotinischer Acetylcholinrezeptoren. Für die Bedeutung weiterer möglicher therapeutischer Ansätze wurden außerdem eine Reihe von genetischen Faktoren sowie zellularpathologische und immunologische Mechanismen beschrieben (Rapp 2009).

Neben dem Alter als stärksten Risikofaktor sind eine niedrige Schulbildung, geringe geistige und körperliche Aktivität, frühe Schädel-Hirn-Traumata, sowie Depressionen als weitere mögliche epidemiologische Faktoren identifiziert worden. Auch vaskuläre Pathologien, wie Diabetes, Bluthochdruck, koronare Herzerkrankungen, Rauchen und Übergewicht korrelieren positiv mit dem Auftreten einer Alzheimer-Erkrankung (Rapp 2009). Der Verlauf dieser Demenzerkrankung beginnt im Allgemeinen im siebten bis achten Lebensjahrzehnt oder später. Nur bei $3 \%$ der Betroffenen manifestiert sich die Krankheit vor dem 65. Lebensjahr. Bereits vor der klinischen Manifestation kommt es in der sogenannten „stummen Phase“ zu neuropathologischen Veränderungen im Gehirn. In der Prädemenz-Phase, die einige Jahre andauert, sind erste kognitive Einbußen feststellbar. Nach zwei bis 15 Jahren endet die eigentliche progressiv verlaufende Demenzphase mit dem Tod (Tölle und Windgassen 2014).

Die Symptome der Alzheimer-Erkrankung manifestieren sich im Laufe der Stadien. Zunächst sind im Rahmen des präklinischen Syndroms die alltagspraktischen Fertigkeiten noch nicht deutlich beeinträchtigt. Es liegen Defizite in der Aufmerksamkeit und den exekutiven Kontrollfunktionen vor, sowie eine Verminderung der Fähigkeit, neue Inhalte zu lernen. Die Symptomatik des frühen Stadiums ist jedoch unspezifisch und muss von depressiven Pseudodemenzen und anderen Ursachen einer leichten kognitiven Störung abgegrenzt werden. Mit fortscheitendem Stadium kommt es zu starken Beeinträchtigungen alltagspraktischer Fertigkeiten und $\mathrm{zu}$ generalisierten Gedächtnisdefiziten bis hin zu ausgeprägten motorischen Störungen mit Apathie (Rapp 2009).

Das klinische Bild des demenzkranken Patienten lässt sich im Einzelnen durch vielerlei Symptome charakterisieren, welche auch auf den Alltag der zahnmedizinischen Praxis Einfluss nehmen. Hierzu zählen beispielsweise Orientierungsstörungen, wodurch sich Patienten in gewohnter Umgebung nicht mehr zurechtfinden. Beeinträchtigt werden diese zusätzlich durch Sehstörungen, welche je nach Befall des visuellen Systems unterschiedlich ausgeprägt sind. Als Folge dessen können sich Raumagnosien, räumliche Alexien, gestörte Auge-Hand-Koordination sowie visuelle Störungen manifestieren. Sprachstörungen zeigen sich zu Beginn der Alzheimer-Erkrankung häufig als anamnestische Aphasien, bei der Wortfindungsschwierigkeiten vorliegen. Bei progressiver Demenz kann es bis zur Auflösung der Sprache, einer globalen Aphasie, kommen (Mielke und Kessler 1994). Eine Kommunikation zwischen Behandler und Patient kann in diesem Stadium nicht mehr stattfinden. Der Alzheimerpatient ist nicht in der Lage, Auskünfte über seine Bedürfnisse und vorliegenden Schmerzsituationen zu geben. Die Gesprächsführung obliegt Angehörigen oder 
Betreuern, welche versuchen, zwischen Patienten und Behandler zu vermitteln und somit auch die ausführende Rolle der intraoralen Pflege nach den Anweisungen des Zahnarztes spielen.

Weitere Symptome sind Veränderungen der Persönlichkeit und des Verhaltens. Diese äußern sich in Beeinträchtigungen der Aktivität und Lokomotion, der Agressionskontrolle, des Tag-NachtRhythmus und des Essverhaltens. Im Rahmen dessen werden charakteristische Muster der Persönlichkeit nivelliert oder vorhandene Charakterzüge werden gesteigert. Bei etwa zwei Drittel aller Erkrankten liegt das depressive Syndrom vor. Diese nicht kognitive psychopathologische Veränderung tritt oft erst später im Verlauf auf und ist bei Patienten mit schweren kognitiven Einbußen schwer zu prüfen (Rapp 2009).

Im fortgeschrittenen Stadium lassen sich fast immer Apraxien nachweisen, die es dem Patienten je nach Form erschweren, alltägliche Bewegungen durchzuführen. So stellt beispielsweise die buccofaciale Apraxie in der Zahnarztpraxis eine besondere Herausforderung dar, da die Betroffenen nicht mehr in der Lage sind, Bewegungen im Gesichtsbereich, wie zum Beispiel das Herausstrecken der Zunge, durchzuführen (Mielke und Kessler 1994).

Das letzte Stadium der Demenz führt häufig zu motorischen Störungen, da es zu einem Befall primärer motorischer Hirnareale kommt. Zeichen hierfür sind spastische Hemiparesen, epileptische Anfälle, Apathie sowie Inkontinenz und Dysphagien (Rapp 2009). Im Rahmen der zahnmedizinischen Behandlung kann es durch diese Symptome zu erheblichen Störungen im Behandlungsablauf kommen. Der Patient kann beispielsweise nicht mehr in der Lage sein, eine kontinuierliche Mundöffnung durchzuführen und eine intraorale Therapie zuzulassen. Alternativplanungen seitens des Zahnarztes müssen vorgenommen werden oder gegebenenfalls eine Überweisung in stationäre Behandlung unter Vollnarkose. Neuropathologisch kommt es zu Amyloidablagerungen über den gesamten Neokortex, in subkortikalen Anteilen und im Hirnstamm. Zentalvegetative Symptome sind nicht selten, die als vegetatives Schwitzen imponieren können (Rapp 2009).

Diagnostiziert wird die Demenz vom Alzheimer-Typ nach Rapp (2009) im klinischen Rahmen über eine Ausschlussdiagnose. Abzugrenzen sind hierbei die vaskuläre Demenz durch eine körperliche und insbesondere neurologische Untersuchung sowie sekundäre Demenzformen, wie beispielsweise bei Vitaminmangelzuständen, endokrinen oder infektiösen Erkrankungen. Zur klinischen Beurteilung gehören außerdem bildgebende Verfahren. Mittels MRT kann eine Darstellung mediotemporaler Atrophien mit einer Spezifität und Sensitivität von $85 \%$ erzeugt werden (Rapp 2009). Zur laborchemischen Diagnose wird der Nachweis des beta-Amyloid und des Tau-Proteins im Liquor, herangezogen. Diagnostische Kriterien wurden zum einen durch die ICD-10, zum anderen durch die NINCDS/ADRDA (National institute of neurological and communicative disorders and strokel Alzheimer's diseases and related disorders assocication) formuliert. Die ICD-10 fordert 
neben der Beeinträchtigung von mindestens zwei kognitiven Funktionen über einen Zeitraum von sechs Monaten das Vorliegen eines schleichenden Beginns mit stetiger Verschlechterung. Durch die wissenschaftlichen Kriterien der NINCDS/ADRDA-Arbeitsgruppe kann differenzialdiagnostisch zwischen dem möglichen, wahrscheinlichen und sicheren Vorliegen einer Alzheimer-Demenz unterschieden werden. Des Weiteren wird eine sichere Diagnose nur durch histopahtologischer Untersuchung und damit in der Regel postmortal gestellt (Rapp 2009).

\subsubsection{Vaskuläre Demenz}

Die vaskuläre Demenz stellt mit einem Anteil von $15 \%$ die zweithäufigste Form der Demenz dar. Bei den über 65-Jährigen besteht eine Prävalenz von 1-4 \%. Ähnlich wie bei der Alzheimer-Demenz korreliert sie mit dem ansteigendem Alter, jedoch in einem geringeren Maße (Schulte-Herbrüggen und Hellweg 2009). Der vaskulären Demenz liegt eine Durchblutungsstörung der Makrozirkulation oder Mikrozirkulation des Gehirns zu Grunde. Weiterhin können auch Gefäßerkrankungen der extrakraniellen Gefäße zu Durchblutungsstörungen führen. Sie stellen ein uneinheitliches System pathologischer Veränderungen und klinischer Symptomkomplexe dar (Schlegel und Neff 2012). Nach Schulte-Herbrüggen und Hellweg (2009) liegt bei der Entstehung vielmehr die Infarzierung von Hirngewebe als eine chronische suboptimale vaskuläre Versorgung vor. Ebenso seien zerebral artherosklerotische Gefäßveränderungen weniger substanziell für die Menge an untergegangenem Hirngewebe, als kleinere oder größere Infarkte.

Die vaskuläre Demenz lässt sich morphologisch anhand der verantwortlichen Gefäßkomponenten einteilen. Dabei stehen Störungen der Makrozirkulation, der Mikrozirkulation, globale Ischämien und die hämorrhagischen Störungen im Vordergrund. Es muss zwischen arteriellen, venösen und mikrovaskulären Ursachen unterschieden werden. Die Hauptgruppe der Zirkulationsstörung stellen die arteriellen Durchblutungsstörungen dar, während venöse Verschlüsse zu hämorrhagischen Infarkten und die mikrovaskulären Verschlüsse zu einem komplexen Gewebssyndrom führen (Tölle und Windgassen 2014).

Pathogenetisch stellen vor allem die Hypertonie, Diabetes, Hyperlipidäme und andere vaskuläre Pathologien, sowie die Hypoxie bei Herzerkrankungen ein erhöhtes Risiko dar. Auch seltenere Gefäßerkrankungen wie Panarteriitis nodosa, Thrombangitis obliterans oder Lupus erythematodes können zur Demenz führen (Tölle und Windgassen 2014). Laut Mielke und Kessler (1994) besteht bei $80 \%$ der Patienten ein Hypertonus oder Nikotinkonsum als Risikofaktor in der Anamnese. Bei Einstellung des Blutdrucks auf hochnormale Werte und Aufgabe des Rauchens habe sich demnach die kognitive Störung bei hypertonen Patienten mit vaskulärer Demenz verbessert (Mielke und Kessler 1994). 
Symptomatisch beginnt die vaskuläre Demenz mit subjektiven Beschwerden, die im Anfangsstadium als „reizbare Schwäche“ beschrieben werden können. Sie ist insbesondere von Müdigkeit, Kopfschmerzen, unsystematischen Schwindel und Verstimmtheit gekennzeichnet (Tölle und Windgassen 2014). Psychische Symptome, wie Affektlabilität und depressive Störungen treten stärker hervor als bei der Alzheimer-Demenz und führen im Endstadium zu Desorientiertheit und motorischer Unruhe bis hin zu ausgeprägten Parkinson-Symptomen des Patienten. Die Ausprägung der Symptome ist bestimmt von der Hirnlokalisation der vaskulären Schädigung und ist bei einzelnen Patienten wechselhaft (Tölle und Windgassen 2014). Schulte-Herbrüggen und Hellweg (2009) beschreiben die Heterogenität und Unspezifität der Symptomatik mit Hinblick auf die unterschiedlichen Unterformen der vaskulären Demenz als eine besondere diagnostische Herausforderung. Bereits die anfänglichen Symptome können Auswirkungen auf die zahnmedizinische Behandlung haben. Reizbare oder depressive Patienten könnten den Belastungen der Behandlung nicht gewachsen sein, was zu einem verfrühten Therapieabbruch oder Verzögerungen im Behandlungsablauf führt und somit Grund für einen Misserfolg der Behandlung darstellen kann. Auch Symptome wie die im Zusammenhang mit der Demenz auftretenden Kopfschmerzen können seitens des Patienten fehlgedeutet und auf den Mund-Gesichtsbereich projiziert werden, was möglicherweise zu einem fehlerhaften Therapieansatz und somit zu einem Versagen der Behandlung führt. Motorische Unruhen und Parkinsonsymptome stellen neben den denkbaren Schwierigkeiten im Behandlungsablauf auch eine Herausforderung bei der Wahl des geeigneten Zahnersatzes dar. Der Patient kann durch die parkinsonbedingten motorischen Störungen, wie Rigor, Tremor, Hypo- oder Akinese nicht mehr in der Lage sein, herausnehmbaren Zahnersatz ein- und auszugliedern sowie eine adäquate Prothesenreinigung oder dentale Pflege durchzuführen. Der Zahnarzt steht somit vor der Herausforderung einen individuell an die Bedürfnisse des Patienten angepassten Zahnersatz zu erstellen. Wie schon in 1.2.1 beschrieben, können Orientierungsstörungen dazu führen, dass Patienten sich in gewohnter Umgebung nicht mehr zurechtfinden, wodurch ein eigenständiger Zahnarztbesuch nicht mehr möglich sein kann.

Zur Diagnosestellung müssen nach Hamann (2012) folgende klinische Kriterien erfüllt sein: Es sollen mindestens zwei kognitive Domänen betroffen und die Fähigkeit des täglichen Lebens beeinträchtigt sein. Dazu müssen im Rahmen von kognitiven Tests zumindest vier kognitive Domänen, nämlich Exekutiv- und Aufmerksamkeitsfunktion, das Gedächtnis, die Sprache und visuospatiale Funktionen, erfasst werden (Hamann 2012). Die klinische Befunderhebung wird vervollständigt, indem vaskuläre Risikofaktoren, wie Diabetes, arterielle Hypertonie, Hyperlipidämie, Alkohol- und Tabakkonsum, erfragt werden. Auch Schlaganfälle, KoronarBypässe, Stent-Einlagen, Vorhofflimmern, Gerinnungsstörungen und arterielle Verschlusskrankheuten werden in der klinischen Vorgeschichte als Risikofaktor erfasst (SchulteHerbrüggen und Hellweg 2009). Durch die darauffolgende neurologische Untersuchung werden alle 
fokalneurologischen Ausfälle erfasst. Zwingender Bestandteil der Diagnostik ist die zerebrale Bildgebung, im Regelfall MRT, um die Lokalisation der vaskulären Läsion zu beurteilen und um Tumore, Atrophien oder einen Hydrocephalus auszuschließen (Schulte-Herbrüggen und Hellweg 2009). Gegenüber der Alzheimer-Demenz ist die vaskuläre Demenz nicht immer sicher abzugrenzen, zumal beide auch kombiniert vorkommen (Tölle und Windgassen 2014).

\subsubsection{Lewy-Körper-Demenz}

Die Demenz mit Lewy-Körperchen stellt mit 15 \% die dritthäufigste Form der Demenz dar. Sie geht klinisch sowohl mit typischen motorischen und vegetativen als auch mit psychiatrischen Symptomen einher (Drach 2009). Genauso wie der Morbus Parkinson gehört die Lewy-Körperchen-Demenz zu den Synukleinopathien. Bei den Lewy-Körperchen handelt es sich um zelluläre Einschlüsse, die Organellen für den Abbau von alpha-Synuklein-Fibrillen darstellen und mit ihrem gehäuften Vorkommen auf eine Störung des alpha-Synuklein-Abbaus hinweisen (Drach 2009). Im Rahmen der Parkinson-Erkrankung sind die typischen histopathologischen zellulären Einschlüsse von allem im Hirnstamm, insbesondere der Substantia nigra, aufzufinden. Bei der Demenz mit Lewy-Körperchen kommt es außerdem zu einem Auftreten der Körperchen im limbischen System und im Neokortex (Schlegel und Neff 2012).

Epidemiologisch sind häufiger Männer als Frauen betroffen, und es kommt zu einem Anstieg der Prävalenz mit zunehmendem Alter (Wallesch und Förstl 2012). Laut Wallesch und Förstl (2012) ist ein typisches Frühsymptom der Lewy-Körperchen-Demenz die Störung des Rapid-Eye-MovementSchlafes, welche sich mit lebhaften Träumen mit starken Bewegungen und Schreien, äußert. Dies kann anderen Symptomen schon um Jahrzehnte vorausgehen. Es wurden Kriterien zur klinischen Diagnose einer Demenz mit Lewy-Körperchen formuliert. Demnach liegt die Voraussetzung darin, dass Funktionseinschränkungen im Alltag vorliegen, die Gedächtnisleistung aber zunächst gut erhalten ist. Insbesondere folgende Symptome sind bei Patienten mit Lewy-Körperchen-Demenz häufiger ausgeprägt als bei Alzheimer-Patienten und für klinische Befunderhebung von Bedeutung. Dazu gehören ein häufigeres Auftreten von extrapyramidal-motorischen Symptomen, fluktuierende kognitive Defizite, visuelle und akustische Halluzinationen und häufig vorkommende Stürze und Synkopen. Liegen mehr als zwölf Monate zwischen Beginn der motorischen und kognitiven Störung, wird nach der 12-Monats-Regel eine Demenz bei Parkinson-Erkrankung diagnostiziert (Wallesch und Förstl 2012). Die vorliegenden Symptome führen $\mathrm{zu}$ - wie unter 1.2.1 und 1.2.2 bereits aufgeführten - Problematiken in der zahnmedizinischen Behandlung. Die kognitiven und motorischen Einschränkungen des Patienten können wie bei der Alzheimer Demenz und der vaskulären Demenz zu Beeinträchtigungen des Behandlungsablaufes und der Kommunikation zwischen Patient und Behandler führen. Der eigenständige Weg zum Zahnarzt kann durch die auftretende Symptomatik visueller Störungen sowie Stürzen und Synkopen nicht mehr durchführbar 
sein. Der Patient ist somit auf die Begleitung Dritter angewiesen, die aufgrund der motorischen Dysfunktionen auch häufig die exekutive Funktion der intraoralen Pflege übernehmen. Die Wahl des Zahnersatzes muss seitens des Zahnarztes dementsprechend auf die individuell vorliegende Symptomatik des Patienten angepasst werden.

Neben der Einbeziehung aller vorliegenden Symptome wird für die klinische Diagnosestellung eine neuropsychologische Untersuchung durchgeführt. Charakteristisch für eine Lewy-KörperchenDemenz sind zur Abgrenzung von einer Demenz bei Parkinson-Erkrankung, nach Wallesch und Förstl (2012), deutliche Fluktuationen kognitiver Leistungen, insbesondere von Aufmerksamkeitsfunktionen in kurz aufeinanderfolgenden neuropsychologischen Untersuchungen. Auch eine Abgrenzung von der Alzheimer-Demenz muss mittels neuropsychologischer Diagnostik erfasst werden. Folgende Kriterien geben in diesem Rahmen Hinweis auf eine Demenz mit LewyKörperchen. Besonders betroffen sind demnach visuokonstruktive Leistungen, die psychomotorische Geschwindigkeit und die Konzeptbildung und Wortflüssigkeit als Frontalhirnfunktion (Wallesch und Förstl 2012). Im Rahmen neurologischer Untersuchungen zur Differenzierung der beiden Demenzformen, stellen deutliche extrapyramidale Symptome, nach Wallesch und Förstl (2012) das wichtigste Unterschiedsmerkmal dar. Als bildgebende Verfahren zur Erfassung einer LewyKörperchen-Demenz kommen CT, MRT, Perfusions-SPECT und die Darstellung präsynaptischer Dopamintransporter zum Einsatz. Mittels Liquordiagnostik lässt sich das alpha-Synuklein in der Zerebrospinalflüssigkeit nachweisen. Bei der Lewy-Körperchen-Demenz ist eine deutliche Verminderung des Synukleins $\mathrm{zu}$ verzeichnen und es stellt somit ein weiteres Unterscheidungsmerkmal zur Alzheimer-Demenz dar (Wallesch und Förstl 2012).

Nach Diagnosestellung verläuft die Krankheit im Schnitt über drei bis sechs Jahre. Verläufe bis zu 20 Jahre wurden ebenfalls beschrieben. Jedoch kann diese Demenzform auch als rasch progrediente Erkrankung verlaufen und nach weniger als 18 Monaten zum Tod führen (Wallesch und Förstl 2012).

\subsection{Problemstellungen in der zahnmedizinischen Behandlung dementer Patienten}

Anhand der beschriebenen Symptome demenzkranker Patienten lassen sich mehrere Problemstellungen in der zahnmedizinischen Behandlung ableiten (s. Abbildung 4). Erstens kann es durch den bei allen Demenzformen auftretende Verlust alltagspraktischer Fertigkeiten und der kognitiven Fähigkeit zu einer Vernachlässigung der Mundhygiene und Inanspruchnahme zahnmedizinischer Leistungen kommen und somit zu einer Begünstigung oraler Pathogene, welche Karies, Gingivitis, Parodontitis und weitere intraorale Pathologien zur Folge hätten. Durch krankheitsbedingt auftretende motorische Störungen kann die adäquate Ausführung der dentalen Pflege nicht mehr selbstständig zu bewerkstelligen sein, da der Patient nicht mehr in der Lage sein 
könnte die Zahnbürste richtig zu halten und koordinierte Putzbewegungen auszuführen. Auch das Ein- und Ausgliedern einer Prothese, sowie deren Reinigung, kann aufgrund der motorischen Einschränkungen impraktikabel werden und Pathologien, wie Prothesenstomatits und Gingivitis begünstigen. Zudem kann eine demenziell bedingte Störung der Hand-Augen-Koordination zu der Nicht-Durchführbarkeit der oralen Hygiene beitragen. Die durch die intraoralen Erkrankungen auftretenden Schmerzen, könnten von dem Patienten durch die demenzbedingte anamnestische oder globale Aphasie nicht richtig geäußert werden und liefen somit Gefahr vom betreuenden Umfeld fehlgedeutet zu werden. Daraufhin kann es zu einer symptomatischen Therapie der durch die Schmerzsituation ausgelöste Verhaltensauffälligkeiten und Lautäußerungen, mit Hilfe von Schmerzmedikamenten und Psychopharmaka kommen, die eigentliche Behebung der Schmerzursache würde jedoch ausbleiben (s. 1.1).

Zweitens ist ein selbstständiger Gang zum Zahnarzt durch das Auftreten von Orientierungs- und Sehstörungen bei demenzerkrankten Patienten, auch bei dem Wunsch nach Behandlung, häufig nicht durchführbar. Auch in Begleitung stattfindende Zahnarztbesuche können bei der betroffenen Patientengruppe durch u. a. das Vorhandensein von Raumagnosien und der räumlichen Alexie zu Unsicherheit und Verwirrtheitszuständen führen, welche sich in einem Abwehrverhalten äußern können. Ein solches Verhalten kann ebenso durch Behandlungsmaßnahmen im Rahmen der zahnärztlichen Therapie erfolgen, da der demenzkranke Patient aufgrund des Aufmerksamkeits- und Gedächtnisdefizites nicht mehr in der Lage sein könnte, den Grund des Arztbesuches und dessen Durchführung kognitiv zu erfassen. Hierbei käme es zu einer Behandlung gegen den Willen des Patienten. Das Selbstbestimmungsrecht des Patienten obliegt jedoch oft einem Vormund oder einem gesetzlichen Vertreter, da die Gesundheitssorge aufgrund der demenziellen Erkrankung häufig abgetreten werden muss (s. 1.1.3). Folge bei den Non-Compliance-Patienten wäre eine Behandlung unter Vollnarkose mit den entsprechenden Risiken (s. 1.1.4). Auch die krankheitsbedinge depressive und aggressive Wesensveränderung kann eine Ursache für das Scheitern einer konventionellen Therapie sein. Eine weitere Problematik im Rahmen der zahnärztlichen Behandlung kann, nach erfolgter intraoraler Versorgung, das geringe Adaptionsverhalten an den erneuerten Zahnersatz sein (s. 1.1.5). Hierbei würde dieser nicht toleriert werden, und ein zahnmedizinischer Benefit bliebe aus oder es käme gar zu einer Verschlechterung der gesundheitlichen Situation. Neben der geringen Adaptionsfähigkeit kann auch die Symptomatik der buccofacialen Apraxie zu einem Scheitern des Zahnersatzes führen, da der Patienten durch die Dysfunktion nicht in der Lage sein könnte, Gebrauch von ihm zu machen (s. 1.2.1). 


\subsection{Zielsetzung der Arbeit}

Zusammenfassend stellt der demenzkranke Patient in vielerlei Hinsicht eine besondere Herausforderung in der Zahnmedizin dar. Aufgrund der breitgefächerten Symptomatik ist der Patient charakterisiert durch seine verminderte kognitive Leistung und Aufmerksamkeit, motorische Störungen, Orientierungsdefizite und Wesensveränderungen, wie aggressives und nicht konformes Verhalten. Durch den folgenden Verlust alltagspraktischer Fertigkeiten kommt es zu einer ganzheitlichen Inanspruchnahme von fremder Hilfsleistung. So obliegt häufig auch die dentale und orale Hygiene dritten Personen, worauf in der zahnmedizinischen Versorgung und Behandlung Rücksicht genommen werden muss. Gesonderte Behandlungsmaßnahmen müssen im Rahmen der zahnmedizinischen Versorgung und des Patientenumgangs umgesetzt werden, um einen Behandlungserfolg zu erzielen. In wie fern die Anfoderungen an diese Patientengruppe in der Praxis berücksicht und umgesetzt werden und welcher Verbesserungsbedarf in der befragten Region besteht, soll mit Hilfe dieser Studie aufgezeigt werden und gegebenenfalls einen Rückschluss auf die Versorgungssituation zulassen.

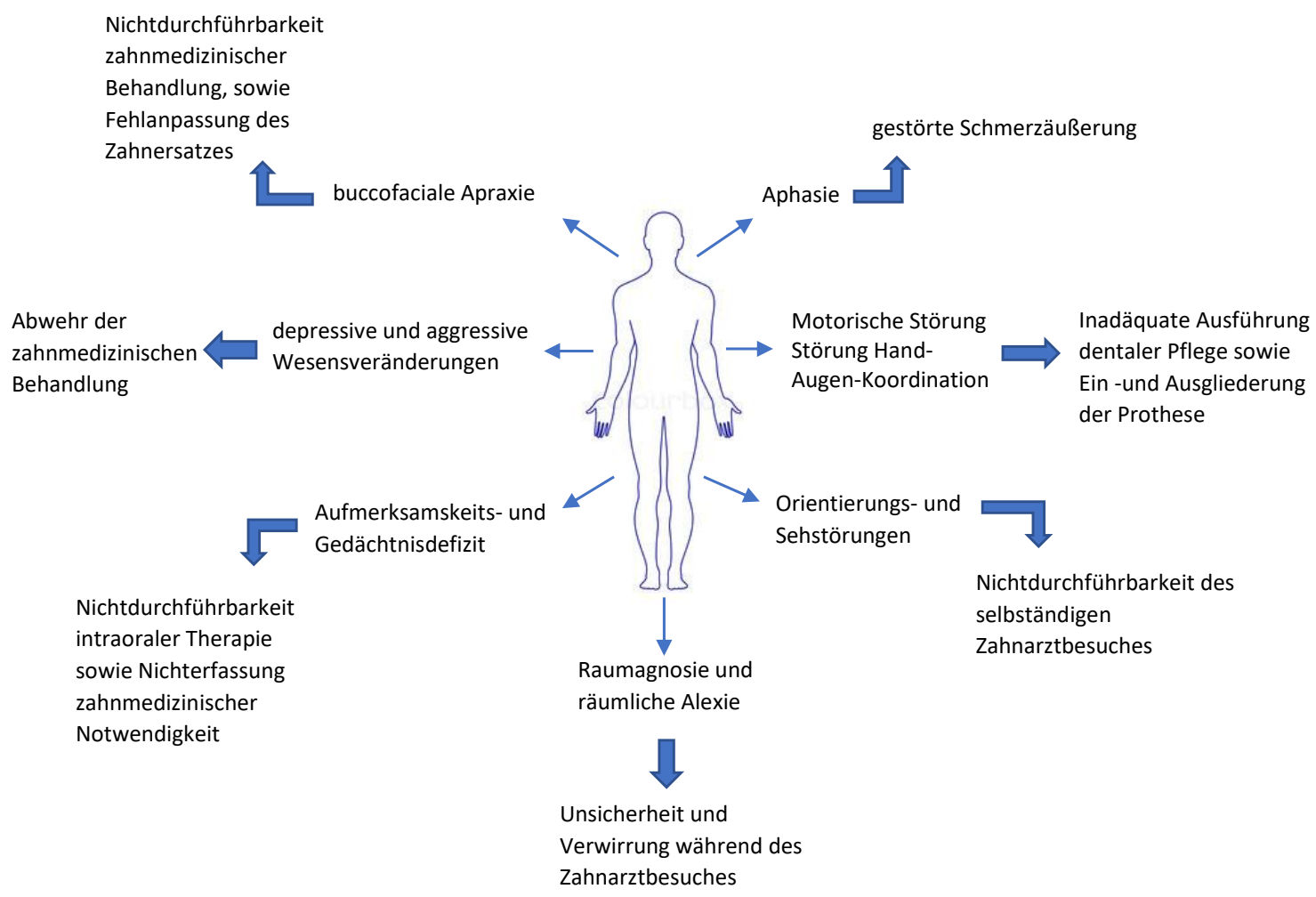

Abbildung 4 Symptomatik und mögliche zahnmedizinische Folgen bei demenzkranken Patienten 


\subsection{Forschungsfragestellungen}

\subsubsection{Fragestellung 1: Allgemeine Informationen zur Praxis}

Anhand einer Fragebogenstudie soll im Rahmen dieser Arbeit Aufschluss darüber gegeben werden, inwiefern die zahnmedizinische Versorgung der Räume Göttingen, Goslar, Northeim und Holzminden auf die besonderen Bedürfnisse dementer Patienten angepasst ist. Um diese Versorgungssituation von allen Seiten $\mathrm{zu}$ betrachten, wurden verschiedene Fragestellungen formuliert. Zunächst sollen allgemeine Angaben zur zahnärztlichen Praxis in Beziehung mit den besonderen Umständen dementer und geriatrischer Patienten gebracht werden. Mit Hilfe des Fragebogens sollen persönliche Angaben wie das Alter und das Geschlecht des Zahnarztes sowie das Abschlussjahr des zahnmedizinischen Staatsexamens ermittelt werden, um mögliche Zusammenhänge dieser Faktoren mit der Behandlungsweise und der Betreuung dementer Patienten zu ermitteln. Auch soll geklärt werden, ob es hinsichtlich der verschiedenen Praxisformen Unterschiede in der Versorgungs- und Betreuungsweise gibt.

Bereits der eigenständige Zahnarztbesuch kann für demente Patienten die erste Komplikation darstellen. Wie unter 1.1.2 beschrieben, liegen zumeist neben der kognitiven Einschränkung typische altersbedingte Begleiterkrankungen und physiologische Alterserscheinungen vor, weshalb sich jene Patienten als multimorbide darstellen. Aufgrund dessen obliegt der Patient meist einer pflegenden Instanz, sei es im häuslichen Umfeld, im stationären Setting oder in einem Pflegeheim. Die Konsultation des Zahnarztes muss somit individuell, je nach Beanspruchbarkeit des Patienten, vorgenommen werden. Diese kann zum einen in Form eines Hausbesuches, einem Besuch im Pflegeheim oder in der Zahnarztpraxis erfolgen. Auswärtige Besuche können dabei unterschiedlich gestaltet werden. Zum einen auf personeller und zum anderen auf materieller Basis. Inwiefern Zahnärzte, mit oder ohne personelle Begleitung, im Raum Göttingen, Goslar, Northeim und Holzminden demente Patienten konsultieren und ob mobile Praxiseinrichtungen genutzt werden, soll mit Hilfe des Fragebogens ermittelt werden und somit die individuellen Versorgungssituationen in den einzelnen Pflegeinstanzen darstellen.

F1.1: Wie werden demenzkranke Patienten in der Praxis konsultiert?

Für demente, multimorbide Patienten, die sich in keiner Pflegeinstanz befinden und einen eigenständigen Zahnartbesuch vornehmen, spielt die Erreichbarkeit der Praxis eine große Rolle. Die mit dem Alter zunehmenden körperlichen Einschränkungen, wie der Sehkraft oder der Mobilität (s. 1.1.2), erfordern zum einen eine deutlich ausgeschilderte und infrastrukturell gut zu erreichende Praxis. Zum anderen kann hier eine Anbindung an öffentliche Verkehrsmittel oder deutlich ausgeschilderte Parkplätze in Praxisnähe wichtig sein. Kurze Wege zur Praxis, sowie ein 
barrierearmer Zugang erleichtern immobilen und körperlich beeinträchtigten Patienten den Weg zu einer zahnmedizinischen Versorgung.

F1.2: Wie ist die Erreichbarkeit für ältere Patienten?

Auch die Ausstattung innerhalb der Praxisräume kann die zahnmedizinische Betreuung dementer Patienten beeinflussen. Faktoren, die den Behandlungsablauf und die Versorgung beeinträchtigen können, sind vor allem die kognitiven Einschränkungen bei vorliegender Demenz, sowie die geriatrie-typische Multimorbidität, die durch die Anwesenheit multimorbiditätsassoziierter Phänomene, wie beispielsweise Schmerz, Immobilität, Sensibilitäts- und sensorische Störungen sowie durch weitere psychische Störungen gekennzeichnet ist (s. 1.1.2). Patienten mit jener Symptomatik sind auf barrierearme Wege innerhalb der Praxis angewiesen, da meist nur noch kurze Strecken zurückgelegt werden können. Eine weitere Problematik bei körperlicher Einschränkung kann ebenfalls eine nicht behindertengerechte Sanitäreinrichtung darstellen. Um während der zahnmedizinischen Behandlung einen möglichst reibungslosen und stressfreien Ablauf zu gewährleisten, kann für diese Patientengruppe der Einsatz von Hilfsmitteln, wie ein Kissen zur Verbesserung der Lagerung oder Zahnbänkchen zum Offenhalten des Mundes, sinnvoll sein. In wie fern die Praxen im befragten Gebiet an die spezielle zahnmedizinische Versorgung und Betreuung diese Patientenklientel angepasst ist und welche Hilfsmittel zum Einsatz kommen, soll mit Hilfe des Fragebogens geklärt werden.

F1.3: Sind Praxen für demenzkranke Patienten ausgestattet?

Besimo und Besimo-Meyer (2015) fordern in ihrer Studie zur Aufzeigung der interdisziplinären Anforderungen für eine verbesserte orale Prävention und Therapie unter anderem, dass der Behandler hinsichtlich der Patientengruppe geschult und ausgebildet ist, um die besonderen Bedürfnisse der gerontopsychiatrisch erkrankten Patienten zu berücksichtigen (s. 1.1). Ob Weiterbildungen des Behandlers und des Personals auf dem Gebiet der Gerodontologie einen Einfluss auf die Versorgungs- und Betreuungssituation dementer Patienten haben und wie häufig diese Weiterbildungsmaßnahmen absolviert werden, soll im Rahmen der Studie ermittelt werden.

Um für den dementen Patienten eine möglichst stressfreie Behandlung zu gewährleisten, können die unter 1.1.3 beschriebenen Prinzipien zur Anwendung kommen, um die Kommunikation zwischen Behandler und Patient zu verbessern. Bei Patienten, die trotz der individuellen Behandlungsprinzipien, keine Compliance aufweisen, kann eine Behandlung unter Vollnarkose in Betracht gezogen werden, wobei eventuelle Folgeschäden berücksichtigt werden müssen (s. 1.1.4). In wie fern solche Strategien, wie beispielsweise die Verwendung von Anschauungsmaterialien, die Gesprächsführung unter Einbeziehung Dritter oder die Überweisung zur stationären Behandlung, zur 
Verbesserung des Behandlungsablaufes in den Zahnarztpraxen im Raum Göttingen, Goslar, Northeim und Holzminden zum Einsatz kommen, soll anhand dieser Fragebogenstudie ermittelt werden. Da die gesonderten Behandlungsstrategien vom normalen Behandlungsablauf abweichen und somit mehr Zeit in Anspruch nehmen können, soll ebenfalls erfragt werden, ob es diesbezüglich Besonderheiten im Praxismanagement, wie beispielsweile spezielle Recall-Strukturen oder im Vorfeld zeitlich länger geplante Behandlungssitzungen für Demente, gibt. Nitschke et al. (2015) fordert in diesem Zusammenhang, dass die Terminvergabe an demente Patienten sensibel erfolgen soll um Rücksicht auf beispielsweise administrative Abläufe, Pflegeprozesse und Bringdienste, sowie Medikamenteneinnahmen der Patienten, zu nehmen (s. 1.1.4).

F1.4: Welche Besonderheiten gibt es in der Weiterbildung und im Praxismanagement auf dem Gebiet der Gerodontologie?

\subsubsection{Fragestellung 2: Versorgung und Behandlung demenzkranker Patienten}

Um dementen Patienten eine individuelle, deren Bedürfnisse entsprechende zahnmedizinische Versorgung zu gewährleisten, müssen gesonderte Maßnahmen und Behandlungsstrategien erfolgen. Wie bereits 1.1.5 zu entnehmen ist, müsse im Rahmen der Therapieentscheidung Einflussfaktoren wie Multimorbidität, verminderte Motorik, Sensorik und kognitive Fähigkeit berücksichtigt werden. Diese Faktoren fließen sowohl bei der Auswahl und der Gestaltung des Zahnersatzes sowie der intraoralen Pflegemaßnahmen als auch bei der hinführenden Behandlung mit ein. Aufgrund der bereits benannten Einflussfaktoren muss die zahnmedizinische Versorgung dementer Patienten individuell auf die besonderen Bedürfnisse angepasst sein. Wöstmann (2003) fordert in diesem Zusammenhang einen leicht handhabbaren, pflegefähigen und robusten Zahnersatz für betagte Patienten um das Kauorgan möglichst lange funktionsfähig zu halten (s. 1.1.5). Da die Adaptionsfähigkeit dieser Patientengruppe an einen neuen Zahnersatz herabgesetzt sein kann, soll in die Therapieentscheidung eine möglichst frühe Umstellung von festsitzenden auf herausnehmbaren Zahnersatz mit angedacht werden um im weiteren Verlauf nur noch kleinere Erweiterungsmaßnahmen vornehmen zu müssen (s. 1.1.5). Ob im Rahmen dieser Behandlungsstrategien auch eine strengere Indikation zur Zahnextraktion gestellt wird und in wie fern die Umstellungsmaßnahmen von festsitzenden auf herausnehmbaren Zahnersatz erfolgen, soll durch die Fragebogenstudie geklärt werden.

F2.1: Welche Besonderheiten gibt es in der Versorgung von demenzkranken Patienten?

Ebenso soll aufgezeigt werden, ob es Unterschiede im Bereich der intraoralen Pflege gibt. Da die motorisch und sensorisch eingeschränkten Patienten häufig nicht mehr in der Lage sind einer adäquaten Mundhygiene nachzukommen und aufgrund dessen meist Dritte zur Prävention und 
Zahnpflege mit einbezogen werden (s. 1.1.3), können spezielle Zahn- und Prothesenpflegemittel sinnvoll sein, welche im Rahmen der Auswertung aufgezeigt werden sollen. Wie unter 1.1 beschrieben, berichtet die Fünfte Deutsche Mundgesundheitsstudie, dass ältere Menschen mit Pflegebedarf eine höhere Karieserfahrung als die nicht betroffene Altersgruppe aufweist und dass jene auch nicht in der Lage seien, eine adäquate Mundpflege eigenständig durchzuführen. Neben der intraoralen Unterversorgung stellt auch die polypharmazeutische Therapie mit geriatrietypischen Medikamenten durch ihre Nebenwirkungen ein Risiko für intraorale Erkrankungen dar (s. 1.1.2). Inwiefern die Zahnärzte im befragten Gebiet intraorale Auffälligkeiten bei dementen Patienten feststellen, soll im Rahmen der Studie aufgezeigt werden.

F2.2: Gibt es intraorale Besonderheiten bei demenzkranken Patienten?

\subsubsection{Fragestellung 3: Beobachtungen bei demenzkranken Patienten in der Praxis}

Um eventuelle kognitive Einschränkungen zu identifizieren und dem Patienten frühzeitig gesonderte Behandlungsmaßnahmen zu ermöglichen, ist es sinnvoll Screeningverfahren anzuwenden. Die unter 1.1.5 aufgeführten Verfahren, der Uhrentest, der Mini-Mental-Status-Test und der DemenzDetektions-Test eignen sich hierbei besonders für den zahnmedizinischen Alltag. Neben der frühzeitigen Identifikation vorliegender Demenz können auch Patienten mit bereits gestellter Diagnose besonderer Beobachtung unterliegen. Vor allem der Mini-Mental-Status-Test dient zur Verlaufskontrolle und kann zur Einschätzung des Schweregrades durchgeführt werden (s. 1.1.5).

Des Weiteren ist bei demenzkranken Patienten darauf zu achten, dass sie immer in Begleitung Dritter zu den Behandlungsterminen erscheinen. Aus juristischen Gründen sind die gesetzlichen Betreuer für die Gesundheits- und Vermögenssorge zuständig, da der Patient aufgrund der kognitiven Einschränkungen nicht mehr in der Lage ist eigenständig Entscheidungen zu treffen (s. 1.1.3).

Anhand der Fragebogenstudie soll aufgezeigt werden, in wie fern demente Patienten im Raum Göttingen, Goslar, Northeim und Holzminden einer besonderen Beobachtung unterliegen und mit welchen Mitteln eine mögliche kognitive Einschränkung identifiziert wird.

F3: Unterliegen demenzkranke Patienten in der Praxis einer besonderen Beobachtung? 


\section{Material und Methoden}

\subsection{Fragebogen}

Um Aufschluss über die Anzahl behandelter Patienten mit einer demenziellen Erkrankung, sowie über deren Betreuung und zahnmedizinische Versorgung zu erhalten, wurde ein spezieller Fragebogen entwickelt. Neben demographischen Informationen (Alter, Staatsexamen, Geschlecht, Praxisform) wurde die Versorgungssituation durch drei übergeordnete Themenschwerpunkte, anhand der Forschungsfragestellungen dieser Arbeit, erfasst:

(1) Allgemeine Informationen zur Praxis (F1.1 bis F1.4)

(2) Zahnmedizinische Versorgung und Behandlung demenzkranker Patienten (F2.1 und F2.2)

(3) Beobachtungen bei demenzkranken Patienten in der Praxis (F3)

Um eine möglichst große Anzahl an Informationen zu erhalten, wurden neben dichotomen Fragen (,ja“ vs. „nein“) Ratings mit 4-/5-stufigen Likertskalen, Häufigkeits- bzw. Prozentangaben, sowie offene Fragen gestellt. Diese werden im Folgenden, geordnet nach den Themenschwerpunkten, dargestellt. Die vollständig formatierte Version des Fragebogens befindet sich im Anhang 6.2 dieser Dissertation.

\subsubsection{Allgemeine Informationen zur Praxis (F1)}

Im ersten Themenschwerpunkt des Fragebogens erfolgten allgemeine Fragen zur (1) Konsultation des Patienten (s. Tabelle 1), sowie zu Informationen über die Praxis, in Bezug auf (2) Erreichbarkeit (s. Tabelle 2) und (3) Ausstattung (s. Tabelle 3). Darüber hinaus mussten Angaben zu (4) Besonderheiten in der Weiterbildung und im Praxismanagement auf dem Gebiet der Gerodontologie, getätigt werden (s. Tabelle 4).

Tabelle 1 Konsultation des Patienten (F1.1)

\begin{tabular}{|c|c|}
\hline Fragestellung: „Wie konsultieren Sie demenzkranke Patienten?“ & Antwortmöglichkeiten \\
\hline „In Ihrer Praxis?““ & „Ja“ vs. „Nein“ \\
\hline „Im Pflegeheim?““ & „Ja“ vs. „Nein“ \\
\hline [Wenn ja]: „Mit personeller Begleitung?“ & „Ja“" vs. „Nein“ \\
\hline „Zu Hause?“ & „Ja“ vs. „Nein“ \\
\hline [Wenn ja]: „Ist dazu eine mobile Praxiseinrichtung vorhanden?“ & „Ja“ vs. „Nein“ \\
\hline
\end{tabular}


Tabelle 2 Erreichbarkeit der Praxis (F1.2)

Fragestellung: „Ist Ihre Praxis für ältere Patienten gut erreichbar?“

Antwortmöglichkeiten

„Hat Ihre Praxis Anschluss an öffentliche Verkehrsmittel?“

„Ja“vs. „Nein“

„Gibt es ausgeschilderte Parkplätze in Praxisnähe?“

„Ja“ vs. „Nein“

„Ist Ihre Praxis gut auffindbar ausgeschildert?“

„Ja“ vs. „Nein“

„Besitzt Ihre Praxis einen barrierearmen Zugang?““

„Ja“ vs. „Nein“

Tabelle 3 Praxisausstattung (F1.3)

Fragestellung: „Ist Ihre Praxis besonders für demenzkranke Patienten ausgestattet?“ Antwortmöglichkeiten

„Ist Ihre Praxis barrierearm?“

„Ja“" vs. „Nein“"

„Besitzt Ihre Praxis behinderten gerechte Toiletten?“

„Ja“ vs. „Nein“

„Benutzen Sie Hilfsmittel, wie z. B. ein Kissen zur Verbesserung der Lagerung?““

„Ja“ vs. „Nein“"

„Verwenden Sie ein Zahnbänkchen (Mundsperre zum Offenhalten des Mundes) bei

,Ja“ vs. „Nein“

funktionell eingeschränkten Patienten?“

„Verwenden Sie weitere, nicht aufgelistete Hilfsmittel?“

offene Frage

Tabelle 4 Weiterbildung und Praxismanagement (F1.4)

Fragestellung: „Welche Besonderheiten gibt es in der Weiterbildung und im Praxismanagement auf dem Gebiet der Gerodontologie?“"

Antwortmöglichkeiten

„Haben Sie auf dem Gebiet der Gerodontologie eine besondere Weiterbildung?“

,Ja“"vs. „Nein“

„Ist Ihr Personal auf dem Gebiet der Gerodontologie besonders weitergebildet?“

„Ja“" vs. „Nein““

„Verwenden Sie im Patientengespräch spezielle Anschauungsmaterialen, wie z. B.

,Ja“ vs. „Nein““

Flyer oder Modelle?“

„Findet das Patientengespräch mit demenzkranken Patienten über Dritte „Ja“ vs. „Nein“

(Pflegepersonal, Angehörige) statt?“

„Können Sie bei Terminen mit demenzkranken Patienten mehr Zeit einplanen?“

„Ja“" vs. „Nein“

„Legen Sie für demenzkranke Patienten im Vorfeld eine bestimmte Recall-Struktur

„Ja“ vs. „Nein“ fest?"

„Gibt es Besonderheiten in der Gesprächsführung mit demenzkranken Patienten?“

offene Frage

\subsubsection{Zahnmedizinische Versorgung demenzkranker Patienten (F2)}

Der zweite Themenschwerpunkt befasste sich mit speziellen Fragen zur zahnmedizinischen Situation demenzkranker Patienten. Um deren (5) zahnmedizinische Versorgung einschätzen zu können, mussten Angaben zum Zahnersatz, der Mundhygienesituation, empfohlenen Mundhygienemitteln und speziellen Behandlungsmaßnahmen, wie z. B. Sedierungen oder Vollnarkosen oder zu prophylaktischer Zahnextraktion, gemacht werden (s. Tabelle 5). In Bezug auf die nicht erkranke Altersgruppe erfolgten anhand von Rating-Skalen eine (6) Einschätzung der intraoralen Situation demenzerkrankter Patienten (s. Tabelle 6). 
Tabelle 5 Besonderheiten in der zahnmedizinischen Versorgung (F2.1)

Fragestellung: „Welche Besonderheiten gibt es in der zahnmedizinischen Versorgung von demenzkranken Patienten?“

Antwortmöglichkeiten

„Stellen Sie bei motorisch eingeschränkten Patienten die Indikation zur Zahnextraktion strenger?"“

„Stellen Sie den Patienten früher von festsitzenden auf herausnehmbaren Zahnersatz um?“

„Empfehlen Sie demenzkranken Patienten eine bestimmte Putztechnik?“

[Wenn ja]: „Welche?“

„Empfehlen Sie demenzkranken Patienten spezielle Mundhygienemittel?“

[Wenn ja]: „Empfehlen Sie motorisch eingeschränkten Patienten eine spezielle Zahnbürste?“،

„Welche Hilfsmittel für die Interdentalraumpflege empfehlen Sie motorisch eingeschränkten Patienten?“

„Empfehlen Sie Mundspüllösungen?““

„Welche Hilfsmittel zur Prothesenreinigung empfehlen Sie?“

„Behandeln Sie demenzkranke Patienten mit niedriger Compliance mit sedierenden Medikamenten?“

[Wenn ja]: „Welche?“

„Behandeln Sie demenzkranke Patienten unter Vollnarkose?“

„Überweisen Sie demenzkranke Patienten für eine stationäre Behandlung unter Vollnarkose?"

„Stehen Sie in Kontakt mit Hausärzten oder anderen Ärzten bei der Behandlung demenzkranker Patienten?“

„Legen Sie Wert auf eine funktionell möglich einfache Prothesengestaltung?“

„Gibt es weitere Besonderheiten in der zahnmedizinischen Versorgung demenzkranker Patienten in Ihrer Praxis?“

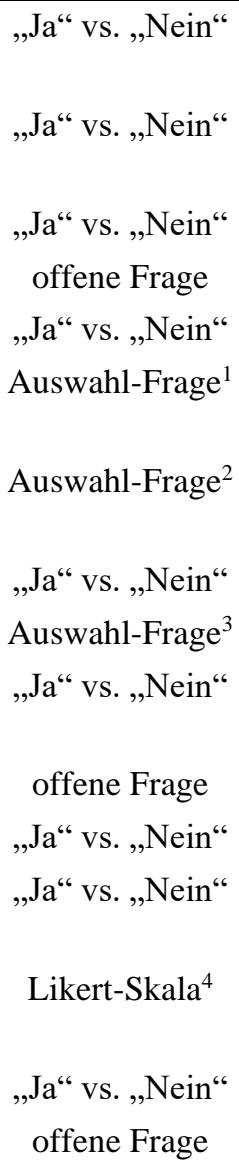

Likert-Skala ${ }^{4}$

„Ja“ vs. „Nein“

offene Frage

Anmerkungen. Die Teilnehmer konnten bei drei Fragen aus vorher definierten Antworten, sowie der Antwort „Sonstige“ wählen. Hierbei waren Mehrfachnennungen möglich. Auswahl-Frage ${ }^{l}=$ elektrische Zahnbürste, Superbrush, Handzahnbürste mit angepassten Griff; Auswahl-Frage ${ }^{2}=$ Zahnseide, Interdentalraumbürsten, Dreikanthölzer; Auswahl-Frage $e^{3}=$ Ultraschallbad, Zahnbürste und Zahnpasta, Reinigungstabletten. LikertSkala $=1$ (,nie“), 2 (,,selten“), 3 (,häufig“), 4 (,,immer“). 
Tabelle 6 Intraorale Besonderheiten (F2.2)

Fragestellung: „Gibt es intraorale Besonderheiten bei demenzkranken Patienten?“

Antwortmöglichkeiten

„Stellen Sie bei demenzkranken Patienten eine Unterversorgung in Bezug auf die

Likert-Skala $^{1}$ nicht betroffene Altersgruppe fest?"

„Stellen Sie bei demenzkranken Patienten eine schlechtere Mundhygiene als bei der

Likert-Skala $^{1}$ nicht betroffenen Altersgruppe fest?"“

„Stellen Sie bei demenzkranken Patienten häufiger intraorale Auffälligkeiten und Erkrankungen fest?“ [Wenn ja]:

„Karies?“

„Ja“vs. „Nein“

„Gingivitis?“

Likert-Skala ${ }^{1}$

„Druckstellen?“

Likert-Skala $^{1}$

„Parodontitis?“

Likert-Skala ${ }^{1}$

„Prothesenstomatitis?“

Likert-Skala ${ }^{1}$

„Xerostomie?“

Likert-Skala ${ }^{1}$

„Hypersalivation?“

Likert-Skala

„Mundschleimhautentzündungen?“

Likert-Skala ${ }^{1}$

„kanzerogene Veränderungen?“

Likert-Skala ${ }^{1}$

Likert-Skala

„Sind demenzkranke Patienten Ihrer Meinung nach in der Lage eine eigenständige

Likert-Skala $^{1}$ adäquate Mundhygiene auszuführen?“"

Anmerkungen. Likert-Skala ${ }^{l}=1$ („,trifft nicht zu“), 2 („,trifft eher nicht zu“), 3 (,,unentschlossen“), 4 (,trifft eher zu“), 5 (,trifft voll zu“).

\subsubsection{Beobachtungen bei demenzkranken Patienten in der Praxis (F3)}

Der letzte Themenschwerpunkt des Fragebogens sollte Aufschluss darüber geben, ob demenzkranke

Patienten einer (7) speziellen Beobachtung in der Praxis unterliegen und ob z. B. Screening-Bögen

oder spezielle Tests zu derer Erfassung genutzt werden (s. Tabelle 7).

Tabelle 7 Spezielle Beobachtung demenzkranker Patienten (F3)

\begin{tabular}{|c|c|}
\hline $\begin{array}{l}\text { Fragestellung: „Unterliegen demenzkranke Patienten in } \\
\text { Ihrer Praxis einer besonderen Beobachtung?““ }\end{array}$ & Antwortmöglichkeiten \\
\hline $\begin{array}{l}\text { „Unterliegen demenzkranke Patienten in Ihrer Praxis einer besonderen } \\
\text { Beobachtung?“ }\end{array}$ & „Ja“"vs. „Nein“ \\
\hline $\begin{array}{l}\text { [Wenn ja]: „Verwenden Sie spezielle Screening-Bögen oder spezielle Tests zur } \\
\text { Erfassung demenzkranker Patienten?“ }\end{array}$ & „Ja“" vs. „Nein““ \\
\hline [Wenn ja]: „Welche?“ & offene Frage \\
\hline „Kommen demenzkranke Patienten in Begleitung Dritter in Ihre Praxis?““ & Likert-Skala $^{1}$ \\
\hline $\begin{array}{l}\text { "Gibt es weitere Besonderheiten in der Beobachtung demenzkranker Patienten in } \\
\text { Ihrer Praxis?“ }\end{array}$ & „Ja“ vs. „Nein“ \\
\hline [Wenn ja]: „Welche?“ & offene Frage \\
\hline
\end{tabular}

Anmerkungen. Likert-Skala ${ }^{I}=1$ (,nie“), 2 (,,selten“), 3 (,,häufig“), 4 (,,immer“). 


\subsubsection{Zusätzliche Fragen}

Abschließend sollte der Behandler zusätzliche Fragen beantworten (s. Tabelle 8 für eine Übersicht). So konnte er anhand einer offenen Frage Stellung zu möglichem Verbesserungsbedarf bei Umgang und Versorgung demenzkranker Patienten nehmen und ggf. sein Interesse an Fortbildungsmaßnahmen auf diesem Gebiet bekunden. Um abschätzen zu können wie viele demenzkranke Patienten in einem Quartal behandelt werden und wie viele von ihnen über 65 Jahre alt sind, wurden abschließend offene Fragen dazu gestellt.

Tabelle 8 Zusätzliche Fragen

Fragestellung

„Wo sehen Sie Verbesserungsbedarf im Umgang und der zahnmedizinischen Versorgung demenzkranker Patienten?“

„Hätten Sie Interesse an einer Fortbildung für den Umgang mit demenzkranken Patienten?"

„Wie viele demenzkranke Patienten werden in Ihrer Praxis schätzungsweis ein einem Quartal behandelt?“

„Wie viel Prozent sind davon über 65 Jahre?“
Antwortmöglichkeiten

offene Frage

„Ja“ vs. „Nein“

Zahl

Prozentsatz

\subsection{Stichprobe}

Im Rahmen der deskriptiven Fragebogenstudie wurden Zahnärztinnen und Zahnärzte im Raum Göttingen, Holzminden, Northeim und Goslar befragt. Insgesamt wurden in diesem Bereich - nach Recherche der jeweiligen Postleitzahlen - 406 approbierte Zahnärzte/innen bei der Landeszahnärztekammer Niedersachsen online aufgelistet, welche einen Fragebogen erhielten. Insgesamt beantworteten $N=119$ Teilnehmer den Fragebogen (Rücklaufquote: 29,3\%), hiervon $31,1 \%$ Frauen $(n=37)$ und 68,9 \% Männer $(n=82)$. Das mittlere Alter der Teilnehmenden betrug $M=52,95(S D=10,55)$. Weibliches Geschlecht korrelierte hierbei positiv mit jüngerem Alter $(r=$ $0,39, p<, 01)$, bzw. später absolviertem Staatsexamen $(r=0,37, p<, 01)$. Für die Gesamtstichprobe wurde das Staatsexamen durchschnittlich im Jahr 1990 absolviert $(M=1990,75, S D=10,42)$. Ansässig waren die Studienteilnehmer in freien Zahnarztpraxen $(n=70,58,8 \%)$ sowie Gemeinschaftspraxen $(n=49,41,2 \%)$. Im Mittel behandelten sie $M=14,77(S D=25,12)$ demenzkranke Patienten im Quartal, davon waren 89,2 \% $\geq 65$ Jahre alt. Teilnehmer aus sonstigen Praxisformen, wie medizinischen Versorgungszentren oder Klinikabteilungen konnten nicht generiert werden. Es wurden selbstständige Zahnärztinnen und Zahnärzte, sowie jene in einem Angestelltenverhältnis, befragt. 


\subsection{Datenerhebung}

Anhand postalisch versendeter Fragebögen wurden deskriptive Daten zum Umgang und zur zahnmedizinischen Versorgung demenzkranker Patienten, erhoben. Den Befragten wurde eine Frist von vier Wochen gesetzt, in welcher der Fragebogen ausgefüllt und zurückgesandt werden sollte. Durch ein beigefügtes Anschreiben wurden die Befragten zum Projekt, sowie zum Datenschutz und rechtlichen und ethischen Aspekten, aufgeklärt (Anhang 6.1). Daraus konnte entnommen werden, dass zu der vorliegenden Befragung ein positives Ethikvotum vorlag ${ }^{2}$ und dass keinerlei Rückschlüsse auf Person und Praxis möglich sein werden. Um die ausgefüllten Fragebögen innerhalb der vorgegebenen Frist zurück zu schicken, wurde ein bereits vorfrankierter Rückumschlag beigefügt.

\subsection{Statistische Auswertung}

Die Daten wurden mit der Statistiksoftware SPSS ${ }^{\circ}$, Version 24, ausgewertet. Ziel der vorliegenden Studie war in erster Linie eine deskriptive Darstellung der zahnmedizinischen Versorgungssituation demenzkranker Patienten. Dementsprechend steht die deskriptive Darstellung der erhobenen Daten mittels beobachteter Häufigkeiten, Prozentwerte, Pearson-Korrelationen $(r)$, Mittelwerte $(M)$ und Standardabweichungen $(S D)$ im Vordergrund. Zusätzlich zur deskriptiven Darstellung sollte in den durch die Forschungsfragestellungen (s. Abschnitt 1.5) definierten Kernbereichen dieser Arbeit explorativ untersucht werden, ob es Unterschiede hinsichtlich der Versorgungssituation zwischen den beiden in dieser Stichprobe (1) vertretenen Praxisformen (alleinige Praxis vs. Gemeinschaftspraxis) und zwischen (2) männlichen vs. weiblichen Behandlern gibt. Abhängig von der Skalierung der entsprechenden Variablen wurden hierzu multiple Chi-Quadrat-Tests $\left(\chi^{2}\right)$, sowie t-Tests $(t)$ mit den 2-stufigen Zwischensubjektfaktoren „Praxisform“ sowie „Geschlecht“ berechnet. Alle Signifikanzen werden auf zweiseitigem Niveau, bei initial festgelegtem Signifikanzniveau von $p<, 05$, berichtet. Aufgrund des deskriptiven Charakters der Studie und der hohen Anzahl an Items im verwendeten Fragebogen wird auf eine Korrektur des Signifikanzniveaus aufgrund der $\alpha$-Fehler Inflation verzichtet.

\footnotetext{
${ }^{2}$ Um im Rahmen der vorliegenden deskriptiven Fragebogenstudie die Datenerhebung und die Datenanalyse durchzuführen, wurde bei der Georg-August-Universität Göttingen ein Ethikantrag gestellt. Dieser Antrag wurde am 28.06 .19 bewilligt.
} 


\section{Ergebnisse}

\subsection{Konsultation demenzkranker Patienten (F1.1)}

Um den Zugang zu zahnmedizinischer Versorgung für demenzkranke Patienten aufzuzeigen, wurde mit insgesamt fünf Items (s. Tabelle 1) nach der Konsultation seitens des Zahnarztes gefragt. 92,4 \% $(n=110)$ der Zahnärzte gaben an, demente Patienten in ihrer Praxis zu behandeln. Eine Konsultation im Pflegeheim wurde von 60,5 \% $(n=72)$ der Befragten durchgeführt, $45,4 \%(n=54)$ davon mit zusätzlicher personeller Begleitung. Demenzkranke Patienten wurden außerdem mit einer Häufigkeit von 39,5 \% ( $n=47)$ von den Zahnärzten zu Hause konsultiert, wobei nur in 8,4 \% ( $n=10)$ eine mobile Behandlungseinrichtung zur zahnmedizinischen Versorgung vorhanden war. Bezogen auf Fragestellung F1.1 behandelt somit die große Mehrheit der Teilnehmer demenzkranke Patienten in der eigenen Praxis, gefolgt von der Behandlung im Pflegeheim und zu Hause (s. Abbildung 5).

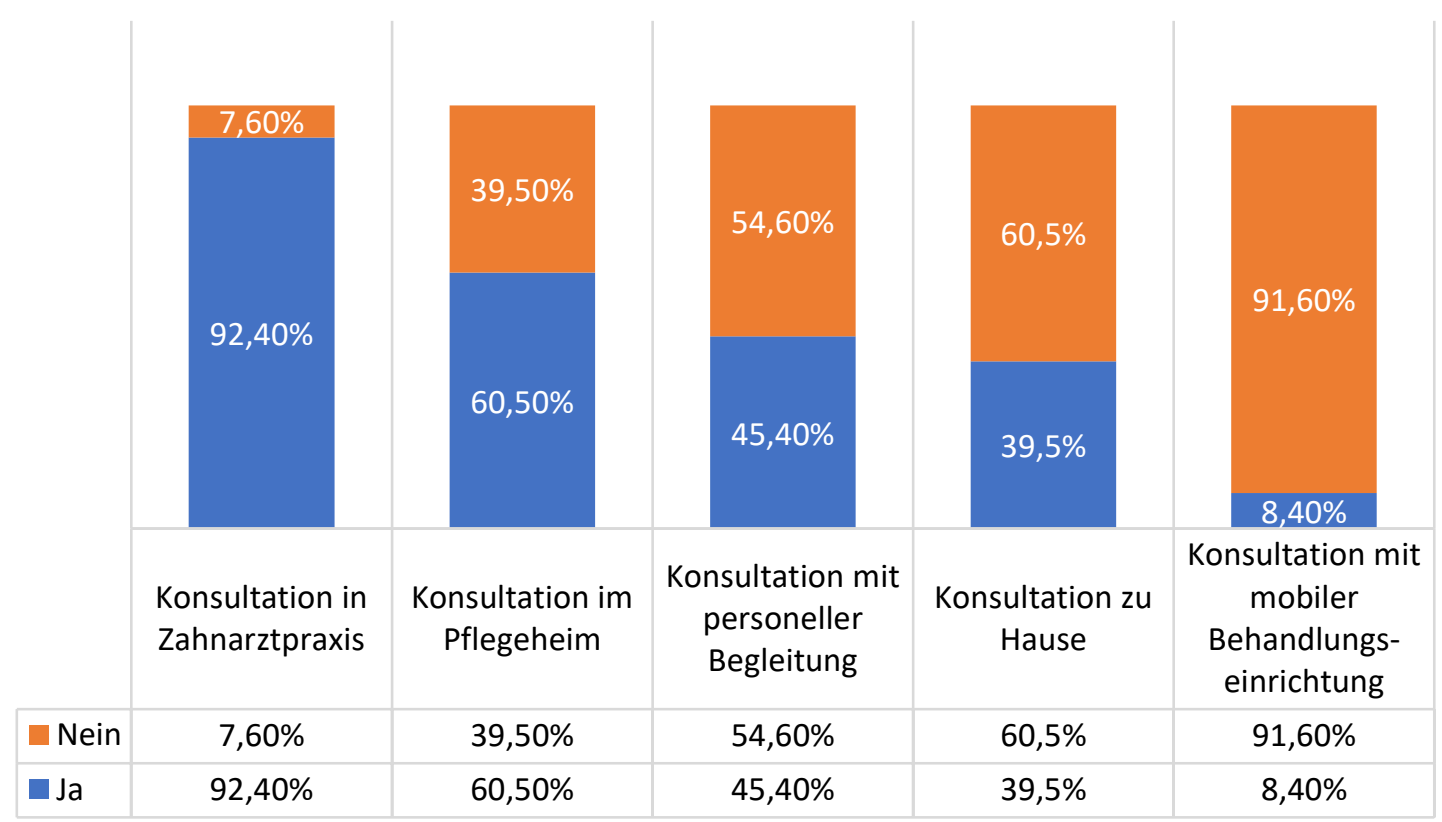

Abbildung 5 Prozentuale Auswertung der „Ja vs. Nein“- Antworten zur Konsultation demenzkranker Patienten

Bei den Items zur Konsultation fanden sich keine Unterschiede zwischen männlichen und weiblichen Behandlern $\left(\chi^{2}(1, N=55-115)\right.$ von $<0,01$ bis $1,86, p$ von, 17 bis $\left., 95, n s\right)$. Auch zwischen beiden in der Stichprobe vorhandenen Praxisformen konnten keine Unterschiede bzgl. der Konsultation von Demenzpatienten gefunden werden $\left(\chi^{2}(1, N=55-115)\right.$ von $<0,01$ bis $1,78, p$ von, 18 bis $\left., 96, n s\right)$. 


\subsection{Erreichbarkeit (F1.2)}

Es sollten vier Fragen über die die Erreichbarkeit der Zahnarztpraxis beantwortet werden (s. Tabelle 2). Die Mehrzahl der Studienteilnehmer gab mit 86,6 \% $(n=103)$ an, Anschluss an öffentliche Verkehrsmittel zu haben. 79,8 \% ( $n=95)$ beschrieben gut ausgeschilderte Parkplätze, 89,9 \% ( $n=$ 107) gaben an, eine gut ausgeschilderte Praxis zu haben. $63 \%(n=75)$ der Praxen wiesen einen barrierearmen Zugang auf. Dementsprechend kann man, bezogen auf Fragestellung F1.2, von überwiegend günstiger Erreichbarkeit der Praxen ausgehen (s. Abbildung 6).

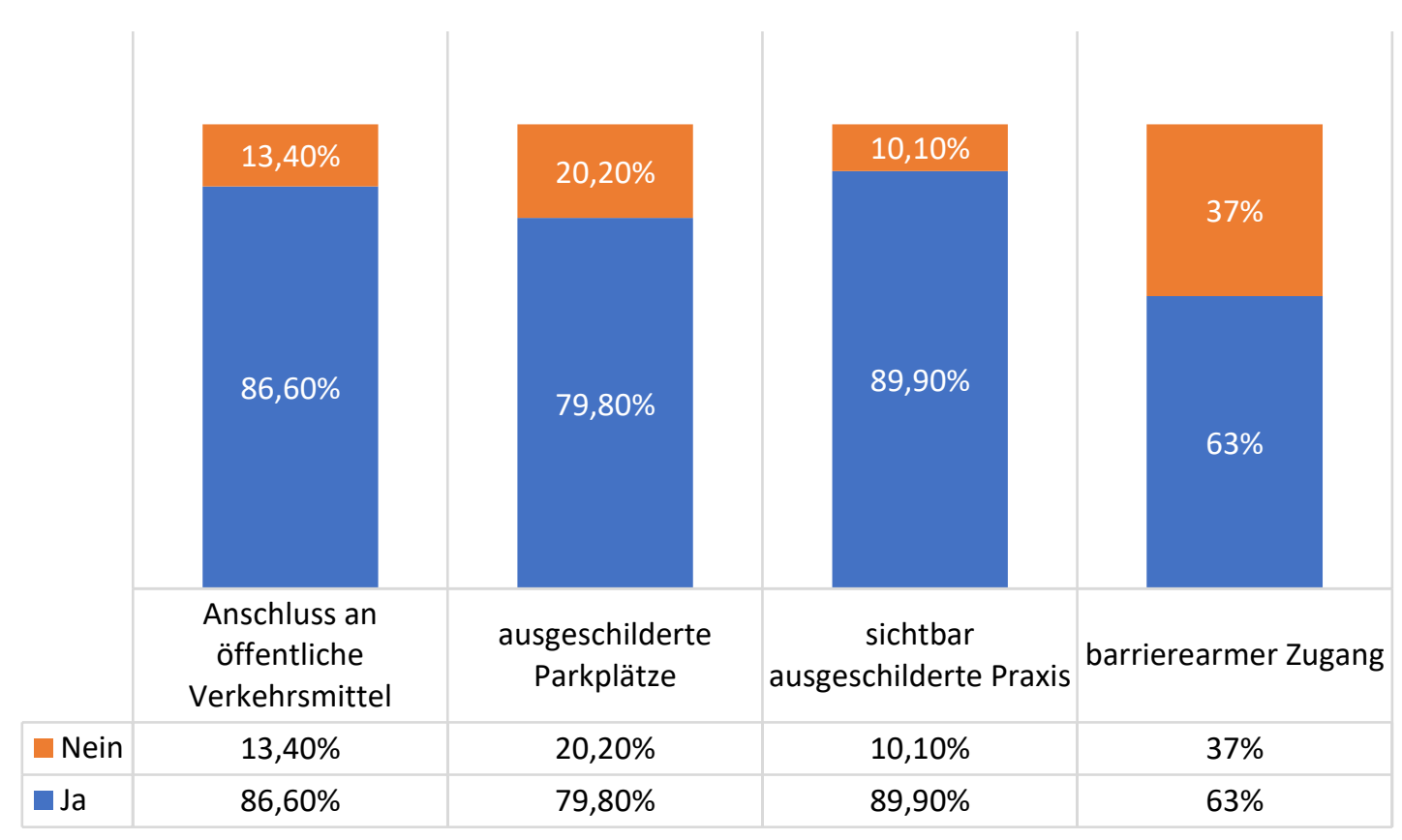

Abbildung 6 Prozentuale Auswertung der „Ja vs. Nein“- Antworten zur Erreichbarkeit der Praxis

Es fanden sich keine Unterschiede zwischen männlichen und weiblichen Behandlern bzgl. der Erreichbarkeit $\left(\chi^{2}(1, N=119)\right.$ von 0,03 bis $2,28, p$ von, 13 bis $\left., 86, n s\right)$. Bei Gemeinschaftspraxen zeigte sich keine bessere Erreichbarkeit im Vergleich zu allein geführten Praxen $\left(\chi^{2}(1, N=119)\right.$ von $0,43$ bis $2,09, p$ von, 15 bis $, 51, n s)$.

\subsection{Praxisausstattung (F1.3)}

Um aufzuzeigen, ob die befragten Zahnarztpraxen auf die besonderen Bedürfnisse von demenzkranken Patienten eingestellt sind, wurden fünf Fragen zur Innenausstattung und zusätzlichem Equipment gestellt (s. Tabelle 3). Die Mehrzahl der Studienteilnehmer gab mit 68,1 \% $(n=81)$ an, eine barrierearme Praxis zu haben. Behindertengerechte Toiletten konnten nur von 31,9 $\%(n=38)$ der Befragten vorgewiesen werden. Um eine bessere Behandlungssituation für demenzkranke Patienten zu schaffen, gaben 83,2\% $(n=99)$ an, Hilfsmittel, wie beispielsweise ein 
Kissen zur Verbesserung der Lagerung, zu nutzen. Bei funktionell eingeschränkten Patienten wurden Zahnbänkchen zum Offenhalten des Mundes nur zu 37,8 \% $(n=45)$ verwendet. Um weitere, nicht aufgelistete Hilfsmittel aufzuführen, konnte eine offene Antwort gegeben werden. Lediglich drei der befragten Studienteilnehmer ergänzten die Antwortmöglichkeiten durch die Angabe der Nutzung eines Metallfingerschutzes, von Bisskeilen oder durch die Unterstützung vom Personal um Kopf und Kiefer des Patienten zu halten. Dementsprechend kann man, bezogen auf F1.3, von einer überwiegend günstigen, an die Bedürfnisse von demenzkranken Patienten angepassten, Praxisausstattung ausgehen. Lediglich in Bezug auf behindertengerecht angepasste Sanitäreinrichtungen lassen sich nur geringe Prozentzahlen verzeichnen (s. Abbildung 7).

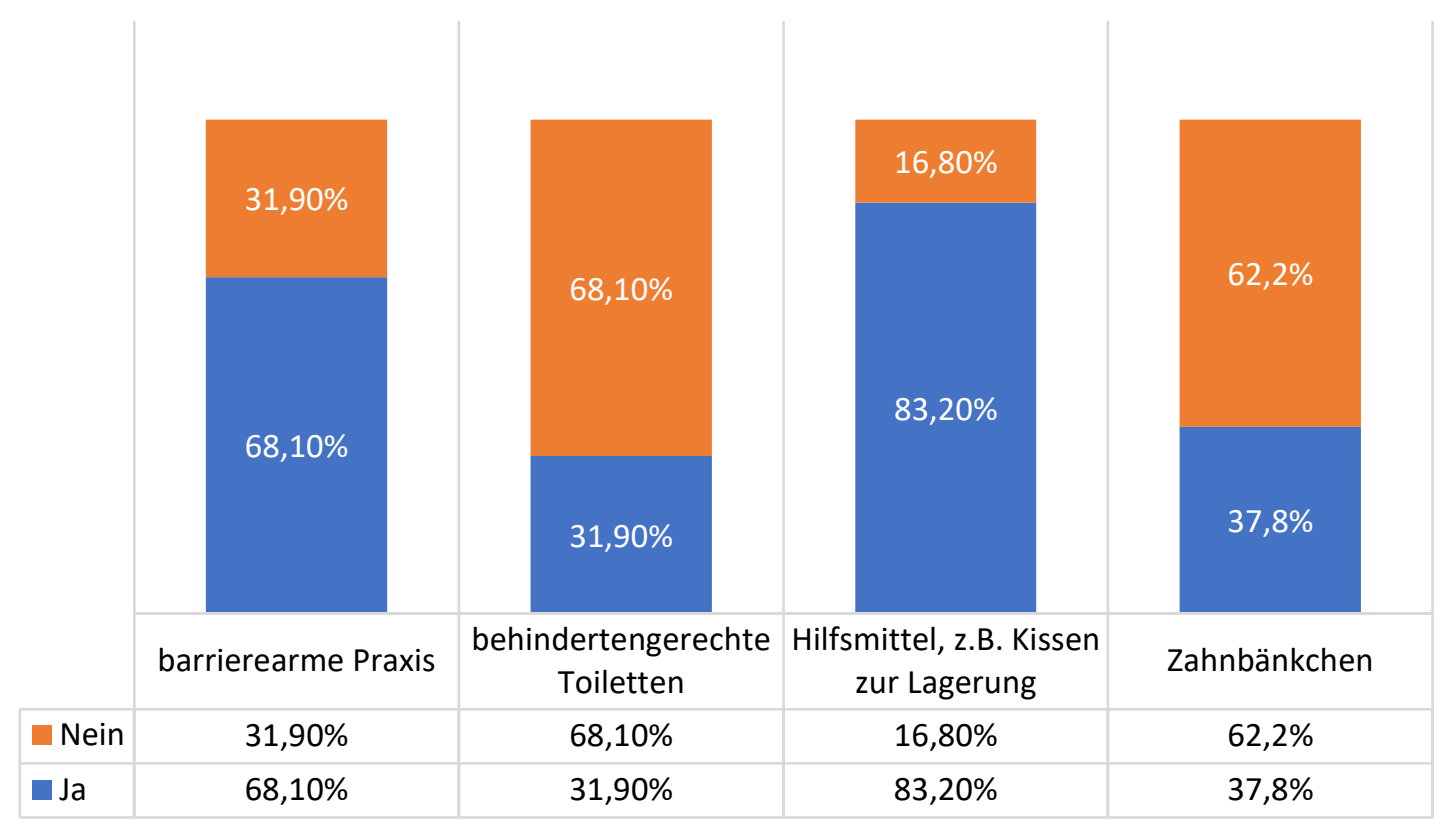

Abbildung 7 Prozentuale Auswertung der „Ja vs. Nein“- Antworten zur Praxisausstattung für demenzkranke Patienten

Im Vergleich zwischen alleiniger Praxisform und Gemeinschaftspraxis ließen sich hinsichtlich der Praxisausstattung zur Frage nach einer behindertengerechten Toiletteneinrichtung Unterschiede aufweisen: Sie war in der Gemeinschaftspraxis in 42,9\% aller Fälle vorhanden, in der alleinigen Praxis nur in $24,3 \%$ der Fälle $\left(\chi^{2}(1, N=119)=4,57, p<, 05\right.$, s. Abbildung 8). Bei den restlichen Fragen konnten keine signifikanten Unterschiede zwischen beiden Praxisformen gefunden werden $\left(\chi^{2}(1, N=115-119)\right.$ von 0,92 bis 2,67, $p$ von ,10 bis ,34, $\left.n s\right)$. Bzgl. männlicher und weiblicher Behandler konnte ein Unterschied gefunden werden: So gaben $58,3 \%$ der Frauen an, auch Zahnbänkchen zu verwenden, während dies nur 30,4 \% der Männer taten $\left(\chi^{2}(1, N=115)=8,11, p<\right.$ ,01, s. Abbildung 8). Bei den restlichen Fragen konnte kein durch das Geschlecht bedingter Unterschied gefunden werden $\left(\chi^{2}(1, N=115-119)\right.$ von 0,267 bis $1,43, p$ von, 23 bis $\left., 61, n s\right)$. 

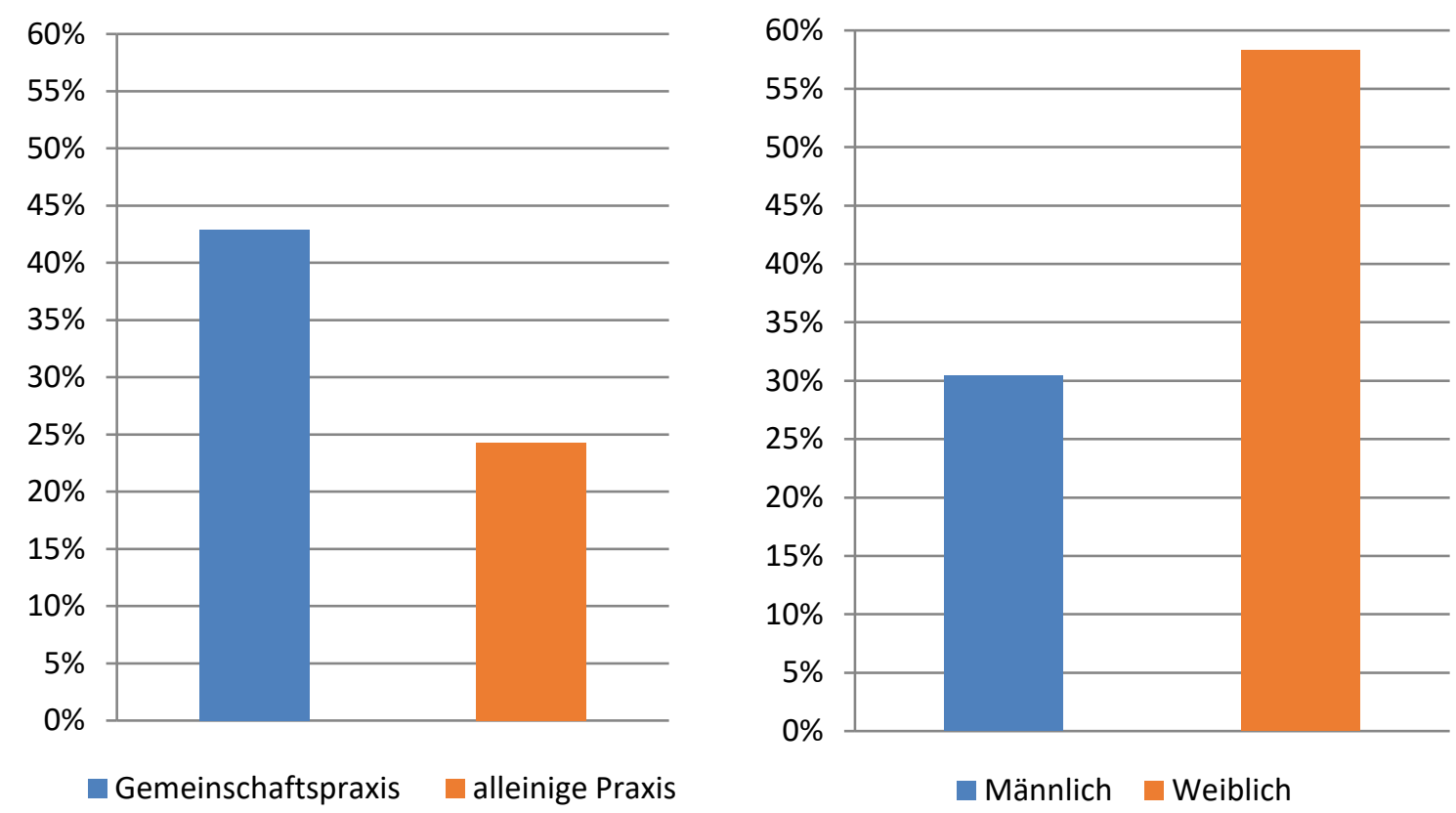

Abbildung 8 Prozentuale Verteilung der behindertengerechten Sanitäreinrichtungen nach Praxisform (links) und der Benutzung von Zahnbänkchen nach Geschlecht (rechts)

\subsection{Weiterbildung und Praxismanagement (F1.4)}

Um Besonderheiten in der Betreuung und der zahnärztlichen Versorgung demenzkranker Patienten zu erfassen, wurde nach Weiterbildungsmaßnahmen seitens des Zahnarztes und des Personals auf dem Gebiet der Gerodontologie gefragt (sieben Items, s. Tabelle 4). Mit einer großen Mehrheit von 89,9 \% ( $n=107)$, haben die befragten Zahnärzte keine Fortbildung auf dem besagten Gebiet absolviert. Auch das Personal wurde mit einer Mehrheit von 92,4\% $(n=110)$ nicht auf diesem Gebiert zusätzlich geschult. Um im Patientengespräch eine bessere Verständlichkeit zu erzielen, werden von 83,2\% $(n=99)$ der Befragten spezielle Flyer oder Anschauungsmaterialen genutzt. Das Gespräch findet zudem bei 92,4 \% ( $n=110)$ aller Teilnehmer über Dritte, wie Angehörige oder das Pflegepersonal statt. Da die Behandlung durch die kognitiven Einbußen des Patienten beeinträchtigt sind, planen 79,8 \% $(n=95)$ der Studienteilnehmer mehr Zeit bei deren Versorgung ein. Nur 19,3\% ( $n=23)$ der Zahnärzte legen zudem im Vorfeld eine bestimmte Recallstruktur zur Überwachung der intraoralen Situation fest. Bezogen auf Fragestellung F1.4 lässt sich somit für die Räume Göttingen, Goslar, Holzminden und Northeim ein sehr geringer Anteil von speziell für demenzkranke und geriatrische Patienten geschultem Fachpersonal verzeichnen. Gesonderte Maßnahmen im Praxismanagement bestehen vor allem in der Gesprächsführung und Zeitplanung (s. Abbildung 9).

Um weitere Besonderheiten in der Gesprächsführung mit dementen Patienten zu erfassen, konnte zudem eine offene Antwort gegeben werden. Als häufigste Ergänzung wurde von den befragten Zahnärzten eine ruhige, langsame und möglichst einfache Gesprächsführung mit Augenkontakt 
empfohlen $(n=10)$. Des Weiteren rieten die Befragten zu einem immer gleichen Behandlungsablauf $(n=2)$ mit Anwesenheit Dritter in Form von Pflegepersonal oder Angehörigen $(n=6)$. Zwei der Studienteilnehmer verwiesen im Bereich der Gesprächsführung auf das Marte Meo Konzept ${ }^{3}$.

Festlegen einer bestimmten Recallstruktur

Einplanung von mehr Zeit während Behandlung

Gesprächsführung über Dritte

Nutzung von Flyern oder Anschauungsmaterialen

Fortbildung Personal

Fortbildung Zahnärzte

\begin{tabular}{|l|}
\hline \\
\hline $19,30 \%$ \\
\hline
\end{tabular}

$20,2 \%$ $79,8 \%$

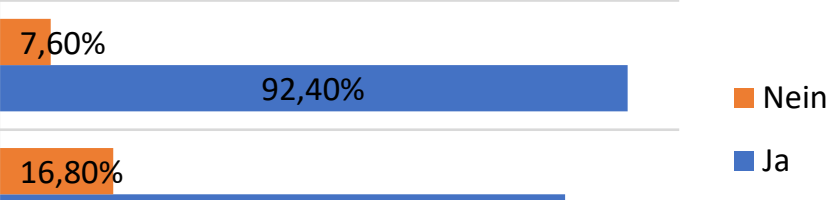

$83,20 \%$

$92,40 \%$

$89,90 \%$

Abbildung 9 Prozentuale Verteilung von „Ja vs. Nein“- Antworten in Hinblick auf weitere Besonderheiten im Praxismanagement und die Bereitschaft zur Weiterbildung

Für die unterschiedlichen Praxisformen konnten keine Unterschiede bzgl. der Weiterbildung und des Praxismanagements gefunden werden $\left(\chi^{2}(1, N=116-117)\right.$ von 0,04 bis $1,47, p$ von, 23 bis $\left., 84, n s\right)$. Auch die Subgruppen der Männer und Frauen unterschieden sich nicht $\left(\chi^{2}(1, N=116-117)\right.$ von 0,03 bis $3,59, p$ von, 06 bis $, 86, n s)$.

\subsection{Besonderheiten in der zahnmedizinischen Versorgung (F2.1)}

Um aufzuzeigen, ob Demenzpatienten durch ihre kognitiven und motorischen Einschränkungen eine andersartige zahnmedizinische Versorgung erfahren, wurden 16 Fragen zur Behandlungsdurchführung und Präventions-, sowie Pflegemaßnahmen beantwortet (s. Tabelle 5). Eine Übersicht der mit „Ja“ vs. „Nein“ zu beantwortenden Fragestellungen ist in Abbildung 10 dargestellt; auf die Ausprägung aller Items wird im Folgenden eingegangen.

\footnotetext{
${ }^{3}$ Das Marte Meo Konzept soll eine planmäßige und verständnisvolle Kommunikation mit unterstützender Hilfe von Videoaufnahmen ermöglichen. Die zunächst im Bereich der Kindererziehung angewandte Methode kann in jeglichen pädagogischen und psychosozialen Arbeitsfeldern zur Anwendung kommen. Dem Patienten und dem Personal, sowie Angehörigen sollen hierbei mit einfacher Sprache und der Unterstützung von Filmsequenzen konkrete und hilfreiche Informationen vermittelt werden (https://martemeopfalz.de/konzept.php, Zugriff am 10.01.18).
} 
möglichst einfache Prothesengestaltung

\section{$15,10 \%$}

stationäre Behandlung unter Vollnarkose

Behandlung unter Vollnarkose

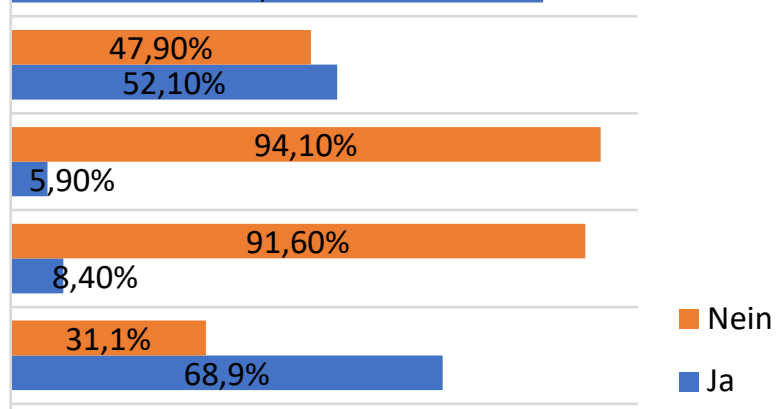

Empfehlung spezieller Mundhygienemittel

Empfehlung spezieller Putztechnik

frühe Umstellung: herausnehmbarer Zahnersatz

strengere Indikation für Zahnextraktion

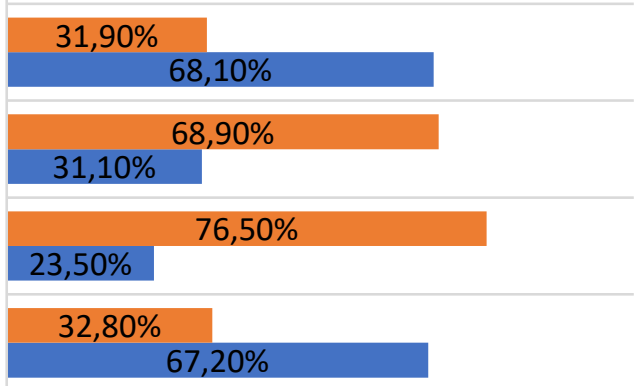

Abbildung 10 Prozentuale Verteilung von „Ja vs. Nein“- Antworten in Hinblick auf Besonderheiten in der zahnmedizinischer Versorgung

Um zu erfassen, ob eine möglichst einfache Mundpflege für Patient, Angehörige oder Personal von den teilnehmenden Zahnärzten gewährleistet wurde, wurden zunächst Fragen nach prophylaktischer Zahnextraktion, Prothesengestaltung und Putztechnik gestellt. 67,2 \% $(n=80)$ der befragten Zahnärzte gaben an, eine Indikation zur Zahnextraktion zur Realisierung einer möglichst einfachen Pflegesituation strenger als bei nicht dementen Patienten $\mathrm{zu}$ stellen. Eine absichtlich frühere Umstellung von festsitzendem auf herausnehmbaren Zahnersatz wurde jedoch nur von 23,5 \% ( $n=$ 28) der Befragten durchgeführt. Zur Verbesserung der Zahnpflege wurden von 31,1\% ( $n=37)$ eine bestimmte Putztechnik empfohlen, in den meisten Fällen durch eine elektrische Zahnbürste $(n=15)$. Zusätzlich sollten Pflegepersonal oder Angehörige dementsprechend mitinstruiert $(n=9)$ werden oder nach der KAI-Technik $(n=3)$ vorgehen, die die Pflege der Kauflächen, Außen- und Innenflächen des Zahnes beschreibt.

Auf die Frage, ob demenzkranken Patienten spezielle Mundhygienemittel empfohlen werden, stimmten $68,1 \%(n=81)$ der Befragten zu. Um einen Überblick zu bekommen, welche Mundhygienemittel bei dieser Patientengruppe besonders häufig zur Anwendung kommen, konnten mehrere vorgefertigte Antworten ausgewählt werden, sowie Freitextangaben gemacht werden (s. Abbildung 11 für eine Übersicht). 


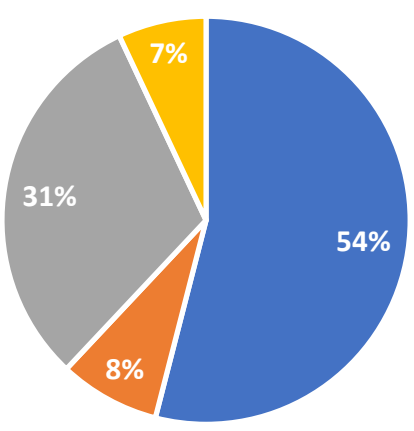

- Elektrische Zahnbürste

- Superbrush

- Handzahnbürste

Sonstige

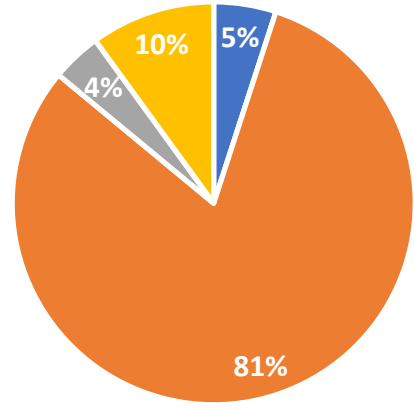

- Zahnseide

- Interdentalraumbürste

- Dreikanthölzer

- Sonstige

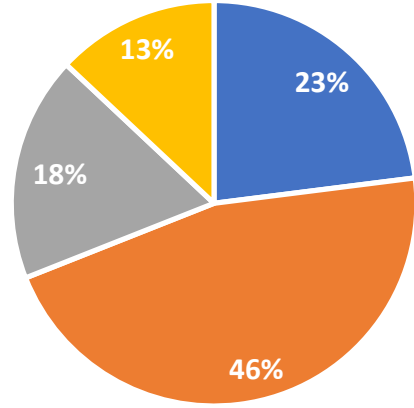

- Ultraschallbad

- Zahnbürste und -pasta

- Reinigungstabletten

- Sonstige

Abbildung 11 Prozentuale Verteilung empfohlener zahnmedizinischer Pflegeartikel für demenzkranke Patienten getrennt nach Hygienemitteln zur dentalen Pflege (links), interdentalen Pflege (mittig) und Prothesenreinigung (rechts)

Von $n=77(64,7 \%)$ Zahnärzten wurde eine elektrische Zahnbürste für motorisch eingeschränkte Patienten empfohlen, $n=44$ (37\%) entschieden sich für eine Handzahnbürste mit angepasstem Griff und $n=11(9,2 \%)$ Teilnehmer rieten zur Superbrush. Darüber hinaus wurde im Freitext von $n=5$ $(4,2 \%)$ Teilnehmern die zusätzliche Anwendung von Mundspüllösungen ergänzt, sowie jeweils einmal eine Zahnbürste mit zwei Putzkörpern, mit Tennisballgriff oder eine Munddusche. Im Bereich der Interdentalraumpflege für motorisch eingeschränkte Patienten entschieden sich die Zahnärzte mit einer Mehrheit von $n=86$ (72,3\%) für Interdentalraumbürsten. Zahnseide wurde von $n=5(4,2 \%)$ Teilnehmern empfohlen, Dreikanthölzer von $n=4$ (3,4 \%). Im Freitext wurde zweimal vormontierte Zahnseide sowie dreimal Tepe easysticks ergänzt, außerdem wurden Mundduschen und Mundspüllösungen zusätzlich empfohlen $(n=3,2,5 \%)$. Mundspüllösungen wurden von 68,9\% ( $n$ = 82) aller teilnehmenden Zahnärzte empfohlen. Um eine effiziente Prothesenreinigung $\mathrm{zu}$ ermöglichen, wurde von $n=90$ befragen Zahnärzten (75,6 \%) das Säubern mit Zahnbürste und Zahnpasta angeraten. $n=45$ (37,8\%) Teilnehmer tendierten zur Reinigung im Ultraschallbad und $n$ $=36(30,2 \%)$ zum Gebrauch von Reinigungstabletten. Im Freitext wurden zusätzlich spezielle Prothesenbürsten $(n=6)$ und Microfasertücher $(n=1)$ sowohl die Reinigung mit Kern- oder Flüssigseife als auch mit Spülmittel $(n=10)$ empfohlen. Des Weiteren sollte von einer professionellen Prothesenreinigung in der Praxis $(n=4)$ Gebrauch gemacht werden.

Da demenzkranke Patienten durch ihre kognitive Einschränkung häufig eine geringe Compliance aufweisen, wurden auch Fragen zum Vorgehen bei Behandlungsverweigerung gestellt. Um eine intraorale Behandlung zu realisieren, gaben $8,4 \%(n=10)$ der Befragten an, sedierende Medikamente bei Nicht-Compliance einzusetzen. Als zum Einsatz kommende Medikamente wurde 
im Freitext Dormicum ( $n=3)$, Lachgas $(n=2)$ und Diazepam $(n=2)$ angegeben. Als weitere Maßnahme bei Therapieverweigerung wurde von 5,9\% $(n=7)$ der befragten Zahnärzte angegeben, eine Behandlung unter Vollnarkose selbstständig durchzuführen. 52,1\% $(n=62)$ überwiesen den Patienten hingegen zu einer stationären Behandlung unter Vollnarkose.

Da demenzkranke Patienten in der Regel in ärztlicher Betreuung sind, wurde zudem gefragt, in welchem Kontakt die befragten Zahnärzte zum betreuenden Hausarzt stehen. Diese Frage konnte von $1=$ „Nie“, 2 = „Selten“, 3 = „Häufig“ bis 4 = „Immer“ beantwortet werden. Im Mittel wurde diese Frage von den Teilnehmern mit $M=2,69(S D=0,82)$ beantwortet. Die meisten Zahnärzte $(n$ $=45,37,8 \%)$ gaben an, dass sie häufig in Kontakt zum Hausarzt stünden, $n=44$ (37\%) selten. Eine geringere Anzahl von $n=20$ (16,8\%) Zahnärzten stünde immer in Kontakt, $n=5$ hingegen nie.

Bei der Anfertigung des herausnehmbaren Zahnersatzes legten mit 84,9\% $(n=101)$ eine große Mehrheit der Zahnärzte Wert auf ein möglichst einfaches und leicht zu handhabendes Prothesendesign. Im Rahmen der offenen Frage mit Freitextantwort wurde zusätzlich ergänzt, dass in der prothetischen Versorgung möglichst wenige Neuplanungen des Zahnersatzes vorgenommen werden sollten und bei Nicht-Vorliegen von Schmerzen keine Behandlung erfolgen soll $(n=2)$. Des Weiteren wurde angegeben, die zahnmedizinische Versorgung individuell nach Vorliegen des jeweiligen Demenzgrades zu gestalten $(n=1)$.

Zusammenfassend lässt sich, bezogen auf Fragestellung F2.1, feststellen, dass Versorgung von demenzkranken Patienten laut den teilnehmenden Zahnärzten Besonderheiten aufweist bzw. besondere Maßnahmen zur Behandlung ergriffen werden. Dies zeigt sich unter anderem bei der Prothesengestaltung, der Empfehlung von Mundhygienemitteln sowie angepasster (wenn nötig auch sedierender) Behandlung bei Patienten mit niedriger Compliance.

Es konnten zudem Unterschiede zwischen alleiniger Praxisform und Gemeinschaftspraxis festgestellt werden (s. Abbildung 12): So gaben 44,7 \% der Zahnärzte in Gemeinschaftspraxen an, demenziell erkrankten Patienten eine bestimmte Putztechnik zu empfehlen, während dies lediglich $24,2 \%$ der Ärzte in alleinigen Praxen taten $\left(\chi^{2}(1, N=113)=5,21, p<, 05\right)$. In der alleinigen Praxis gaben 93,9\% der Teilnehmer an, auf eine funktionell möglichst einfache Prothesengestaltung Wert zu legen. Dieser Wert fiel mit $81,3 \%$ in der Gemeinschaftspraxis geringer aus $\left(\chi^{2}(1, N=113)=4,43\right.$, $p<, 05)$. Generell war die Bereitschaft von Zahnärzten, in einer Gemeinschaftspraxis sedierende Medikamente einzusetzen (17,1 \% vs. 4,3\%) bzw. Patienten unter Vollnarkose zu behandeln (10,4 $\%$ vs. $2,9 \%$ ), erhöht, die Unterschiede zwischen den Praxisformen verfehlten in beiden Fällen jedoch knapp die Signifikanz $\left(\chi^{2}(1, N=116-117)=2,77-3,79, p\right.$ von ,051 bis ,096, $\left.n s\right)$. Männliche Behandler gaben in 8,6 \% der Fälle an, demenzerkrankte Patienten unter Vollnarkose zu behandeln, während dies $0 \%$ der weiblichen Behandler berichteten - auch dieser Unterschied verfehlte knapp die Signifikanz $\left(\chi^{2}(1, N=116)=3,22, p=, 073, n s\right)$. 


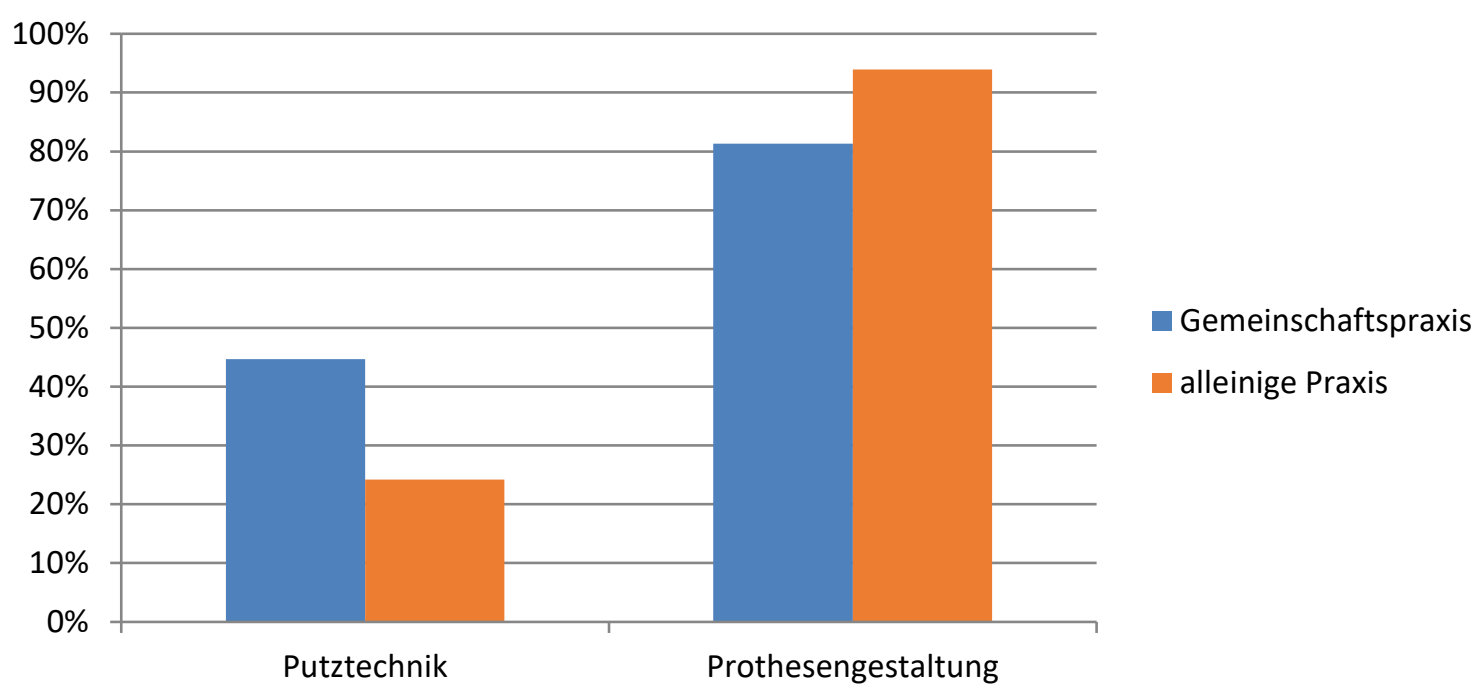

Abbildung 12 Prozentuale Angaben zur Putztechnik und Prothesengestaltung nach Praxisform

\subsection{Intraorale Besonderheiten bei demenzkranken Patienten (F2.2)}

Mit Hilfe des Fragebogens sollte erfasst werden, ob sich bei demenzkranken Patienten im Vergleich zu ihrer nicht erkrankten Altersgruppe intraorale Auffälligkeiten feststellen lassen (für eine Übersicht aller Items s. Tabelle 6). Neben allgemeiner Einschätzung einer möglichen Unterversorgung und

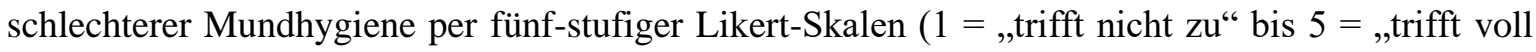
zu“), wurden Zahnärzte neben dem reinen Auftreten um die Einschätzung neun spezifischer intraoraler Auffälligkeiten und Erkrankungen mittels fünf-stufiger Likert-Skalen gebeten. Abschließend schätzten die Teilnehmer auf derselben Skala die Fähigkeit von Demenzpatienten ein, eine eigenständig adäquate Mundhygiene auszuführen.

Eine mögliche Unterversorgung von demenzerkrankten Patienten wurde von den Teilnehmern mit $M=3,44(S D=1,13)$ tendenziell zwischen $3=$,,unentschlossen“ und $4=$,trifft eher zu“ beantwortet. Eine schlechtere Mundhygiene wurde mit $M=4,19(S D=0,87)$ tendenziell als $4=$,trifft eher zu“ bewertet. Mit einer Mehrheit von 75,6 \% $(n=90)$ bejahten die Studienteilnehmer zudem, dass bei dementen Patienten häufiger intraorale Auffälligkeiten festgestellt werden konnten, als bei nicht dementen Patienten.

Die Einschätzung spezifischer intraoraler Auffälligkeiten und Erkrankungen bei demenzerkrankten Patienten ist in Abbildung 13 zusammengefasst. Insgesamt zeigte sich, dass lediglich die Auftretenswahrscheinlichkeit von Hypersalivation $(M=2,59, S D=0,82)$ und kanzerogene Veränderungen $(M=2,04, S D=0,94)$ von den teilnehmenden Zahnärzten als nicht erhöht eingeschätzt wurde, sowie Xerostomie tendenziell mit $3=$, unentschlossen“ $(M=3,12, S D=0,95)$. Alle übrigen intraoralen Auffälligkeiten und Erkrankungen wurden von den Teilnehmern bei demenziell erkrankten Patienten als wahrscheinlicher auftretend eingeschätzt ( $M$ von 3,45 bis 4,29). 
Die höchste Wahrscheinlichkeit wurde hierbei für Gingivitis angegeben $(M=4,29, S D=0,70)$, gefolgt von Parodontitis $(M=4,08, S D=0,83)$ und Karies $(M=4,06, S D=0,85)$.

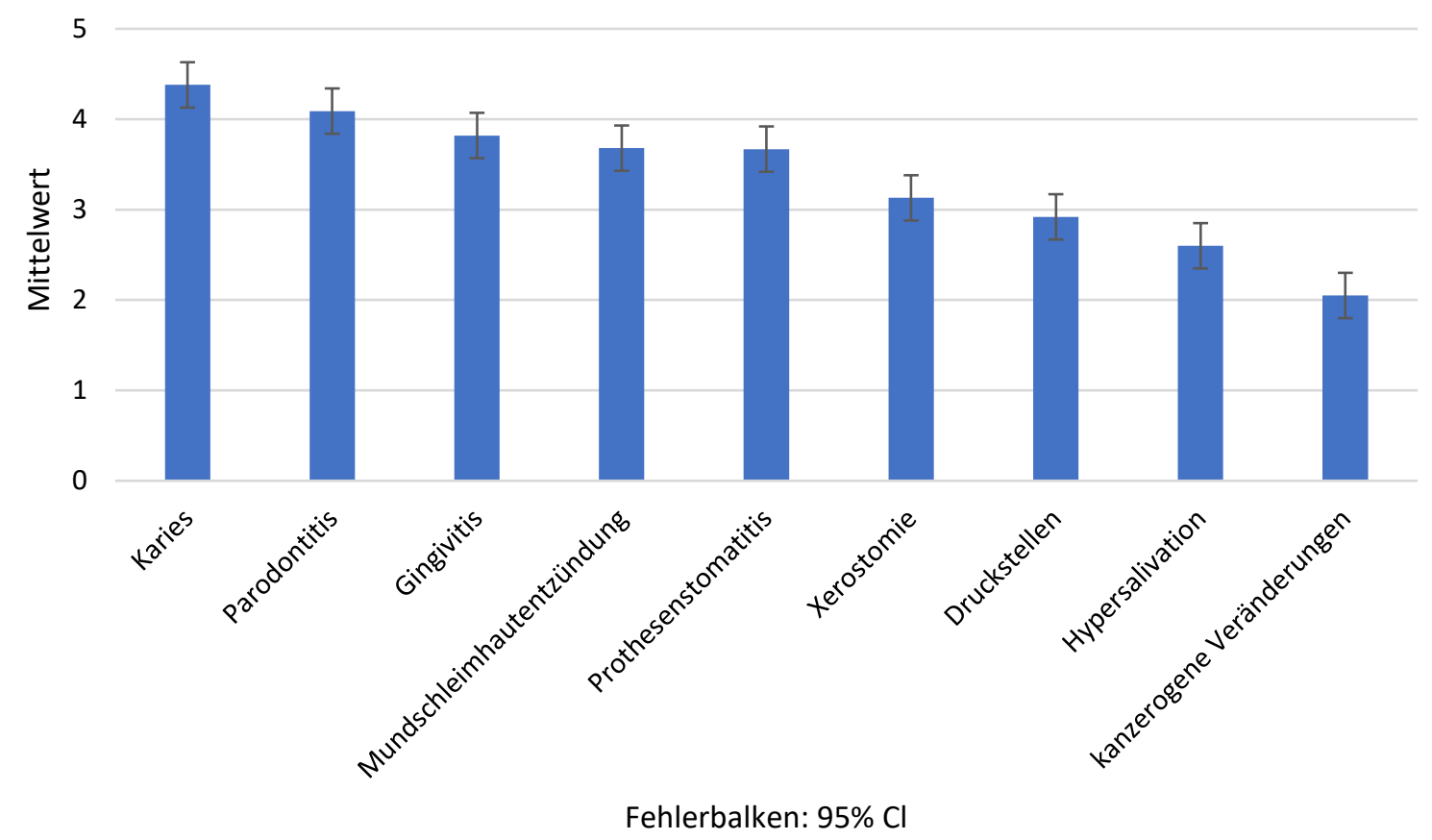

Abbildung 13 Intraorale Auffälligkeiten und Erkrankungen bei demenziell erkrankten Patienten

Um Ursachen für das vermehrte Auftreten intraoraler Erkrankungen bei demenzkranken Patienten aufzuzeigen, sollten die Zahnärzte zusätzlich eine Aussage darüber treffen, ob diese Patientengruppe nach eigenen Ansichten in der Lage sei, eine eigenständige Mundhygiene durchzuführen. Diese Frage wurde mit $M=2,06(S D=0,86)$ tendenziell mit $2=$,trifft eher nicht zu“ beantwortet.

Bezogen auf Fragestellung F2.2 lässt sich somit feststellen, dass laut Einschätzung der teilnehmenden Zahnärzte intraorale Besonderheiten bei demenzkranken Patienten vorliegen, sowohl was Unterversorgung, schlechte Mundhygiene, Auffälligkeiten und Erkrankungen als auch die nicht mehr eigenständig durchführbare, adäquate Mundhygiene als mögliche Ursachen für die gefundenen Erkrankungen angeht.

Bzgl. der Einschätzung intraoraler Besonderheiten konnte ein Unterschied zwischen männlichen und weiblichen Behandlern identifiziert werden: So gaben weibliche Behandler an, bei demenzkranken Patienten häufiger Parodontitis festzustellen $(M=4,34, S D=0,94)$, im Vergleich zu männlichen Behandlern $(M=3,97, S D=0,76, t(104)=2,149, p<, 05$, s. Abbildung 14). Signifikante Unterschiede zwischen beiden Praxisformen bei der Einschätzung intraoraler Besonderheiten konnten für keines der verwendeten Items festgestellt werden. 


\section{5}

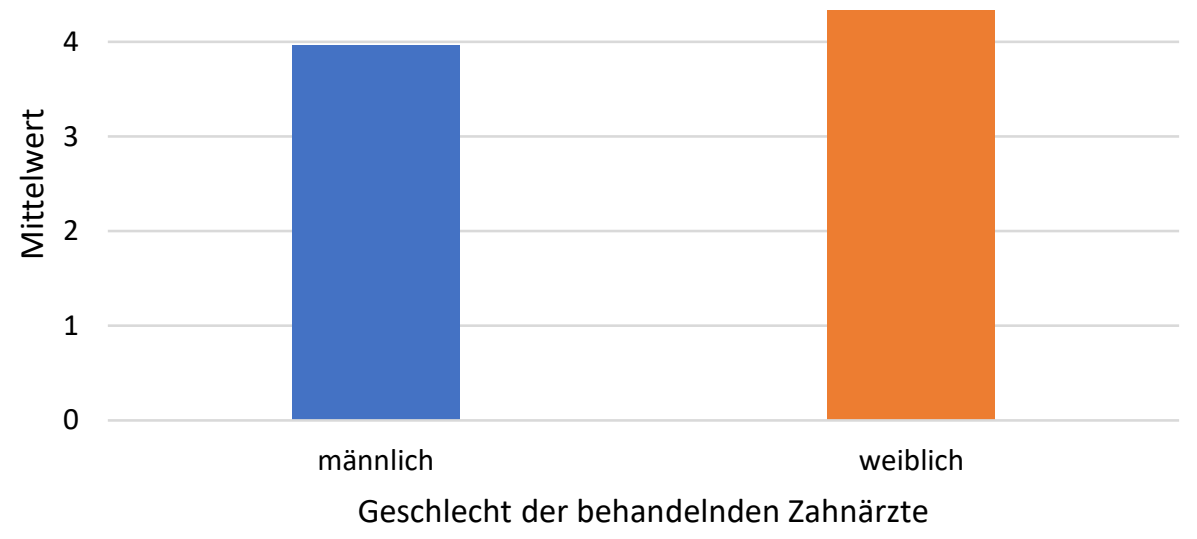

Fehlerbalken: $95 \% \mathrm{Cl}$

Abbildung 14 Einschätzung der Häufigkeit von Parodontitis bei durch männliche und weibliche Behandler

\subsection{Beobachtung demenzkranker Patienten in der zahnmedizinischen Praxis} (F3)

Es wurde mittels sechs Items erfragt, ob die Patientengruppe Demenzerkrankter einer besonderen Beobachtung in der zahnmedizinischen Praxis unterliegt (s. Tabelle 7). Eine Übersicht der mit „Ja“ vs. „Nein“ zu beantwortenden Fragestellungen ist in Abbildung 15 zusammengefasst.

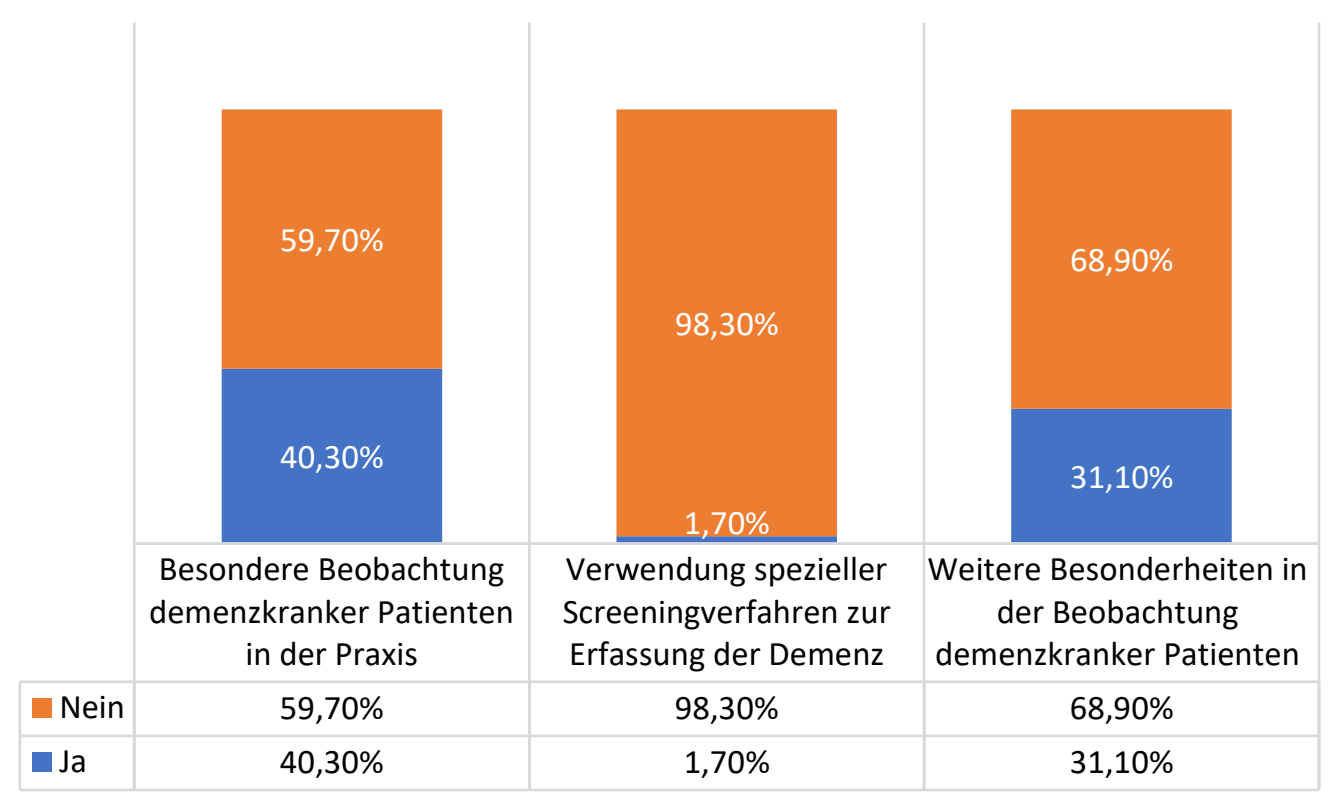

Abbildung 15 Prozentuale Verteilung der „Ja- vs. Nein"- Antworten in Hinsicht auf die besondere Beobachtung demenzkranker Patienten 
Die Mehrheit der Befragten $(59,7 \%, n=71)$ gab hierbei an, dass demenzkranke Patienten keiner besonderen Beobachtung in der Praxis unterliegen. Lediglich 1,7\% $(n=2)$ berichteten, spezielle Demenztests und Screening-Bögen zur Erfassung der Erkrankung einzusetzen. Ein Zahnarzt gab im Freitext an, den Uhrentest bei Verdacht auf Demenz zur Diagnosestellung heranzuziehen. Hierbei handelt es sich um einen Schnell-Screening-Test, im Rahmen dessen Defizite in der räumlichen Vorstellungskraft und in der Fähigkeit des Problemlösens aufgedeckt werden sollen. Der Patient wird dabei aufgefordert innerhalb von zwei bis fünf Minuten das Zifferblatt einer Uhr aufzuzeichnen und eine vorgegebene Uhrzeit einzutragen. Die Auswertung erfolgt mit Hilfe von sechs verschiedenen Scores, welche zur Beurteilung eines möglichen kognitiven Defizits herangezogen werden. ${ }^{4}$ Die Frage, ob es Besonderheiten bei der Beobachtung demenzranker Patienten in der eigenen Praxis gibt, wurde von $68,9 \%(n=82)$ der Befragten verneint.

Die Häufigkeit einer Begleitung Dritter während der zahnärztlichen Konsultation konnte von $1=$ „nie“, 2 = „selten“, 3 = „häufig“ bis 4 = „,immer“ eingeschätzt werden. Die Teilnehmer gaben die Wahrscheinlichkeit mit $M=3,43$ ( $S D=0,56$ ) tendenziell zwischen $3=$ häufig" und $4=$,immer" an. Die Teilnehmer konnten zudem im Freitext weitere Besonderheiten bei der Beobachtung von demenzkranken Patienten angeben. Jeweils eine teilnehmende Person berichtete von (1) besonderer Dankbarkeit/Freundlichkeit, (2) Kooperation/Sprechstunde mit einem Pflegeheim, (3) Beobachtung nachlässiger Mundhygiene, (4) Akzeptabler Mundhygiene, sowie (5) ein reduziertes Spektrum der Behandlungsmöglichkeiten je nach Beweglichkeit und Mitarbeit.

Eine besondere Beobachtung demenzkranker Patienten bezogen auf Fragestellung F3 konnte in der vorliegenden Befragung nicht festgestellt werden. Tests zur Abklärung von Demenz kommen faktisch nicht zum Einsatz.

Weibliche Behandler gaben mit 54,3\% signifikant häufiger als männliche Behandler (32,1\%) an, dass demenzkranke Patienten in ihrer Praxis einer besonderen Beobachtung unterliegen würden $\left(\chi^{2}(1, N=116)=5,07, p<, 05\right)$. In Gemeinschaftspraxen wurde mit 53,2 \% vs. $29 \%$ (alleinige Praxis) ebenfalls signifikant häufiger angegeben, dass demenzkranker Patienten einer besonderen Beobachtung unterlägen $\left(\chi^{2}(1, N=116)=6,90, p<, 01\right)$.

\footnotetext{
${ }^{4}$ https://www.pflege.de/leben-im-alter/krankheiten/demenz/test/uhrentest/, Zugriff am 19.07.18
} 


\subsection{Zusätzliche Fragen}

Eine Übersicht der zusätzlichen Frage ist in Tabelle 8 (vgl. Kapitel 2.1.4, S. 26) dargestellt ${ }^{5}$. Auf die Frage, wo ein Verbesserungsbedarf im Umgang und in der zahnmedizinischen Behandlung von demenzkranken Patienten bestünde, konnte mit einem Freitext geantwortet werden. Als häufigster Verbesserungsbedarf wurde die mangelnde Schulung des Pflegepersonals im Altenheim angesehen $(n=28)$. Des Weiteren sollten die Patienten in regelmäßigeren Abständen zur professionellen Zahnreinigung in der Praxis erscheinen $(n=2)$ und eine höhere finanzielle Unterstützung $(n=6)$ für die Umsetzung zahnmedizinischer Maßnahmen erfolgen. Auch eine Spezialisierung von Zahnarztpraxen auf diese Patientenklientel $(n=2)$ wurde nahegelegt sowie eine vermehrte Möglichkeit zur Behandlung unter Vollnarkose in den Krankenhäusern $(n=1)$. Interesse an einer Fortbildung zum Thema „Umgang mit demenzkranken Patienten“ wurde von 51,3\% $(n=61)$ der Teilnehmer bejaht.

${ }^{5}$ Für die absoluten Zahlen zur Anzahl demenzkranker Patienten und dem Prozentsatz $\geq 65$ Jahre siehe Stichprobenbeschreibung. 


\section{Diskussion}

\subsection{Konsultation demenzkranker Patienten (F1.1)}

Hinsichtlich der Konsultation demenzkranker Patienten, gab die Mehrheit von 92,4 \% ( $n=110)$ der Studienteilnehmer an, demenzkranke Patienten in der Praxis zu konsultieren. Aus dieser Angabe lässt sich entnehmen, dass die Nachfrage im Bereich der zahnmedizinischen Versorgung bei demenzkranken Patienten sehr hoch ist. Die unter 1.1 aufgeführte Fünfte Deutsche Mundgesundheitsstudie beschreibt ebenfalls den hohen Bedarf an zahnmedizinischer Behandlung von pflegebedürftigen Menschen. Um dem, auch durch den demografischen Wandel bedingten, erhöhten Bedarf im Bereich der Gerodontologie nachzukommen, findet die Konsultation dieser Patientengruppe hauptsächlich in der Zahnarztpraxis statt. Als einen Grund dafür geben Baumgartner et al. (2015) an, dass sich Zahnärzte infolge des Drucks aus der Praxistätigkeit und der Freizeitgestaltung nicht in der Lage fühlen, in Institutionen lebende Patienten aufzusuchen. Beklagt wurden ebenfalls die mangelnde Ausbildung, die technische Ausrüstung, verbunden mit der geringen Rentabilität mobiler Ausrüstungen, sowie die geringe Nachfrage seitens der Pflegeheime (Baumgartner et al. 2015).

Es wurde von 60,5\% $(n=72)$ der Befragten angegeben, zahnmedizinische Behandlungen in Pflegeheimen durchzuführen. In Bezug auf die unter 1.1 angeführte Studie von Knabe und Kram (1997), sowie die SÄVIP-Studie (Hallauer et al. 2005), welche eine zahnmedizinische Unterversorgung von Pflegeheimbewohnern beschreiben, war die deutliche Mehrheit der konsultierenden Zahnärzte nicht zu erwarten. Anhand der Angabe der befragten Zahnärzte kann jedoch kein Rückschluss auf die zahnmedizinische Versorgung im befragten Gebiet gezogen werden, da weder die Häufigkeit der Heimbesuche noch die Anzahl versorgter Patienten erfragt wurde. Es lässt sich lediglich feststellen, dass im Raum Goslar, Northeim, Holzminden und Göttingen eine hohe Bereitschaft der Zahnärzte besteht, Patienten im Pflegeheim zu konsultieren. Weniger als die Hälfte der Befragten gaben an, zusätzlich personelle Begleitung bei den Pflegeheimbesuchen zu haben. Begründbar ist dies zum einen damit, dass ein zeitlicher und finanzieller Mehraufwand für das Personal umgangen werden soll, insbesondere, wenn Hausbesuche außerhalb der Arbeitszeit stattfinden. Zum anderen sollen zusätzliche Umstände im Bereich der zahnmedizinischen Fachangestellten vermieden werden, da diese entsprechendes, unter anderem gerostomatologisches Fachwissen vermittelt bekommen müssen, wie z. B. in Form von Zertifikaten (Nitschke und Kaschke 2011). Des Weiteren kann angenommen werden, dass bei den erforderten zahnmedizinischen Arbeiten im Altersheim häufig kein zusätzliches Personal benötigt wird, da jene Besuche in erster Linie dazu geeignet seien sollten, sich ein Bild von der Situation zu machen und nur risikofreie Behandlungen fachgerecht durchzuführen. Für invasive Behandlungen sollte dann der Transport in eine geeignete Einrichtung erfolgen (Ludwig 2016). 
Im Gegensatz dazu, wurden Hausbesuche bei pflegebedürftigen Patienten nur von 39,5\% $(n=47)$ der Befragten durchgeführt. Kretsch (2016), gab mit Hilfe einer vergleichbaren Studie zum häuslichen Setting von Nitschke et al., 2005, eine ähnliche Quote von 30,5 \% für die zu Hause besuchten Patienten an. Dies kann zum einen durch die geringe und somit nicht wirtschaftliche Honorarleistung der gesetzlichen Krankenkassen erklärt werden (Dettbarn-Reggentin und Reggentin 2016), zum anderen, weil das häusliche Versorgungssystem ein primär reaktives System ist, da Zahnarztpraxen lediglich auf die Nachfrage des Patienten reagieren (Kretsch 2016). Um eine häusliche Versorgung dementer Patienten zu realisieren, gaben $8,4 \%(n=10)$ der Befragten an, über eine mobile Praxiseinrichtung zu verfügen. Diese geringe Anzahl kann zum einen damit begründet werden, dass sie in der Anschaffung und Wartung mit hohen Kosten zwischen 2.000 - 10.000 Euro verbunden sind (Ludwig 2010), je nach technischer Ausstattung mit einem Gewicht von bis zu 24 kg (Kretsch 2016) in der Handhabung Probleme darstellen können und sie andererseits bei geringer Inanspruchnahme und fehlender Routine zu einem erhöhten und somit unwirtschaftlichen Zeitaufwand beitragen (Ludwig 2010). Des Weiteren wird auf juristischer Ebene kein Unterschied gemacht, ob die Behandlung unter idealen technischen Aspekten in der Zahnarztpraxis oder unter eingeschränkten Möglichkeiten bei Hausbesuchen durchgeführt wurde, sofern es zu Komplikationen kommen sollte (Ludwig 2010). Aus diesem Grund könnten Zahnärzte von der Verwendung mobiler Einheiten ebenfalls Abstand nehmen. Zusammenfassend lässt sich anhand der Ergebnisse erkennen, dass die zahnmedizinische Versorgung von Pflegebedürftigen nicht ausreichend strukturiert und flächendeckend gewährleistet ist, wie auch Nitschke und Kaschke (2011) in einer bundesweiten Gesundheitsforschung ermittelte.

In Bezug auf die Praxisform und auf das Geschlecht konnten zur Fragestellung, wie demenzkranke Patienten konsultiert werden, keine signifikanten Unterschiede gefunden werden. Hinsichtlich des Einsatzes mobiler Zahnarztpraxen hätte man annehmen können, dass sich Gemeinschaftspraxen aufgrund der gemeinsamen Kostenlast und den steuerlicher Vorteile (Nuri 2015) häufiger dazu entschließen in deren Anschaffung zu investieren. Jedoch könnten aufgrund der geringen Rentabilität solcher mobilen Praxen (Baumgartner et al. 2015) auch Gemeinschaftspraxen im befragten Gebiet davon Abstand genommen haben.

\subsection{Erreichbarkeit (F1.2)}

Für geriatrisch erkrankte Patienten, welche selbstständig die Zahnarztpraxis aufsuchen, stellt der physische und psychische Aufwand die Praxis zu erreichen, einen der häufigsten Gründe für ein Nichterscheinen dar (Bär et al. 2009). Nitschke et al. (2015), gibt ebenfalls als einen der häufigsten Gründe einer Nichtinanspruchnahme zahnärztlicher Leistungen die Gebrechlichkeit und fehlende Mobilität der Senioren an. Im Rahmen der Studie gaben die Teilnehmer zu durchschnittlich 79,8 \% 
an, eine gute Erreichbarkeit für geriatrische Patienten vorzuweisen. Es kann davon ausgegangen werden, dass in den befragten Gebieten der Grund eines Nichterscheinens des Patienten und einer daraus möglichen intraoralen Unterversorgung in den meisten Fällen nicht auf eine schlechte Praxiserreichbarkeit zurückzuführen ist. Diese Einschätzung wurde jedoch lediglich aus Sicht der Zahnärzte getätigt, sodass die tatsächlichen Umstände zur Erreichbarkeit der Praxis für den Patienten in diesem Zusammenhang nicht als günstig oder ungünstig zu beurteilen sind. Ob die mögliche intraorale Unterversorgung aus Patientensicht mit der Praxiserreichbarkeit in Zusammenhang steht, muss in einer weiterführenden Studie untersucht werden.

\subsection{Praxisausstattung (F1.3)}

Laut Bär et al. (2009) hat die Behaglichkeit in der Praxis einen entscheidenden Einfluss auf die Inanspruchnahme zahnmedizinischer Leistungen. Als beeinflussende Faktoren, geriatrischen, immobilen Patienten eine qualitätsorientierte Behandlung zu gewährleisten, fordern Nitschke et al. (2015) die Anpassung des Warte- und Empfangsbereich, sowie des Weges zum Behandlungszimmer, an die Bedürfnisse dieser Patientengruppe. Die Mehrheit der befragten Zahnärzte gab an, über eine barrierearme Praxis zu verfügen und somit diesen Bedürfnissen gerecht zu werden. Behindertengerechte Sanitäreinrichtungen konnten im befragten Gebiet nur zu 31,9 \% ( $n=38)$ erfasst werden. Diesbezüglich ist ein Verbesserungsbedarf zu verzeichnen, da behindertenrechte Toiletten als ein beeinflussender Faktor zur Gewährleistung einer bedürfnisgerechten zahnmedizinischen Behandlung geriatrischer Patienten anzusehen sind (Nitschke et al. 2015). Im Vergleich der jeweiligen Praxisform konnte festgestellt werden, dass Gemeinschaftspraxen häufiger behindertengerechte Toiletten aufwiesen als allein geführte Praxen. Dies könnte durch die finanziell günstigere Situation dieser Praxisform (Nuri 2015) und der somit möglichen Mehrinvestition und dadurch besseren Ausstattung erklärt werden.

Die deutliche Mehrheit der befragten Zahnärzte gab an, Hilfsmittel zur Verbesserung der Behandlungssituation zu verwenden. Bär et al. (2009) sowie Nitschke et al. (2015) plädieren für den Einsatz entsprechender Hilfsmittel, um eine qualitätsorientierte und für den Patienten möglichst stressfreie zahnmedizinische Behandlung zu gewährleisten. Um herauszufinden, welche Hilfsmittel zum Einsatz kommen, wurde als Antwortvorschlag zum einen nach der Verwendung von Zahnbänkchen gefragt, zum anderen konnten weitere Hilfsmittel durch eine offene Antwortmöglichkeit ergänzt werden. $\mathrm{Zu}$ verzeichnen war eine nur geringe Nutzung von Zahnbänkchen, welche nach der Aufschlüsselung nach dem Geschlecht deutlich häufiger von den weiblichen Teilnehmern genutzt wurde. Inwiefern sich der Einsatz von Hilfsmitteln zwischen Zahnärztinnen und Zahnärzten während der Behandlung unterscheidet und ob Frauen generell öfter dazu neigen unterstützende Maßnahmen zur Schonung des Patienten zu nutzen, muss anhand 
weiterführender Studien untersucht werden. Nur wenige Studienteilnehmer ergänzten die Frage durch die Angabe weiterer zum Einsatz kommenden Hilfsmittel. Dies kann entweder mit der mangelnden Bereitschaft oder dem zeitlich verbundenen Mehraufwand den Fragebogen auszufüllen verbunden sein, da offene Fragen in der Regel seltener beantwortet werden, als geschlossene (Barth 1998), oder zum anderen damit, dass generell nur eine geringe Auswahl an unterstützenden Mitteln genutzt wird. Zusammenfassend lässt sich anhand der Ergebnisse feststellen, dass die Praxisausstattung im Raum Göttingen, Goslar, Holzminden und Northeim für geriatrische Patienten überwiegend günstig ist. Bezüglich der behindertengerechten Sanitäranlangen sind jedoch Verbesserungsmaßnahmen erforderlich.

\subsection{Weiterbildung und Praxismanagement (F1.4)}

Besimo und Besimo-Meyer (2015) fordern hinsichtlich der Zielgruppe geriatrischer Patienten, dass der Behandler geschult und ausgebildet sein soll um auf die besonderen Bedürfnisse der gerontopsychiatrisch erkrankten Patienten einzugehen. Mit einer großen Mehrheit haben die befragten Zahnärzte im Raum Göttingen, Goslar, Holzminden und Northeim keine Weiterbildung auf dem besagten Gebiet absolviert. Ähnliche Rückstände in der Aus- und Weiterbildung auf dem Bereich Gerodontologie zeigten Nitschke et al. (2004), mit ihrer Studie „Zur Etablierung der Seniorenzahnmedizin in der zahnärztlichen Ausbildung" auf. Demnach wurde in nur $19,4 \%$ der befragten universitären Einrichtungen eine eigenständige einsemestrige Vorlesung zur Seniorenzahnmedizin angeboten. Laut Approbationsordnung sei noch keine spezielle Ausbildung der Studierenden im Fach Seniorenzahnmedizin gefordert (Nitschke et al. 2004). Bereits die studentische Ausbildung kann somit der Forderung von Besimo und Besimo-Meyer (2015) nicht gerecht werden und spiegelt den eigentlich hohen Bedarf an späteren Fortbildungsmaßnahmen wieder. Die geringe Teilnahme der befragten Zahnärzte an Weiterbildungsmöglichkeiten kann zum einen an den bestehenden Eigenkosten zum Erwerb eines Curriculums im Bereich der Seniorenzahnmedizin zurückzuführen sein, zum anderen auf das mangelnde Interesse oder den nicht ersichtlichen Weiterbildungsbedarf auf diesem Gebiet. So bekundete im Rahmen der Fragebogenstudie lediglich eine knappe Mehrheit von 51,3\% $(n=61)$ das Interesse an einer Fortbildung. Es ist davon auszugehen, dass im Bereich der Gerodontologie hinsichtlich der zahnärztlichen Aus- und Weiterbildungsmaßnahmen noch große Rückstände vorliegen und somit ein Verbesserungsbedarf besteht. Auch auf personeller Ebene lassen sich Rückstände im Bereich von Fort- und Weiterbildungsmaßnahmen mit Hilfe der Fragebogenstudie verzeichnen. Hierbei könnten sich ähnliche Gründe anführen lassen, welche bereits auf der mangelnden Inanspruchnahme der Zahnärzte beruhen. 
Hinsichtlich des auf demenzkranke Patienten abgestimmten Praxismanagements ließen sich mittels der Fragebogenstudie positive Angaben im Bereich der Kommunikation zwischen Behandler und Patient verzeichnen. Wie in der Literatur unter anderem von Bär et al. (2009) gefordert, setzt die Mehrheit der befragten Zahnärzte Flyer und Anschauungsmaterialen zur Verbesserung des Verständnisses ein. In den meisten Fällen werden zusätzlich Dritte, wie z. B. Angehörige oder Pfleger in das Patientengespräch mit einbezogen. Dies ist nicht nur zur Verbesserung der Kommunikation zwischen Behandler und Patient hilfreich, sondern auf juristischer Ebene vorgeschrieben, da der gesetzliche Betreuer für die Gesundheits- und Vermögenssorge anwesend sein muss, da der Patient durch seine kognitive Einschränkung nicht mehr in der Lage ist, eigenständige Entscheidungen $\mathrm{zu}$ treffen (Ludwig 2016). Hinsichtlich der gesonderten Behandlungsmaßnahmen zum verbesserten Verständnis und der Kommunikation kann bereits ausgegangen werden, dass die Behandlung dementer Patienten mehr Zeit in Anspruch nimmt, als die der nicht betroffenen Zielgruppe. Dementsprechend vergeben die befragten Zahnärzte im Raum Göttingen, Goslar, Holzminden und Northeim mit einer großen Mehrheit im Vorfeld längere Behandlungstermine an die betroffene Patientengruppe. Somit wird der Großteil der Studienteilnehmer der in der Literatur geforderten Einplanung von einem zeitlichen Mehraufwand während der Behandlung dementer Patienten gerecht, um eine Verbesserung der zahnmedizinischen Versorgung zu erzielen und die gesonderten Behandlungsmaßnahmen berücksichtigen zu können (Bär et al. 2009; Nitschke und Kaschke 2011). Die von Bär et al. (2009) zusätzlich empfohlene Vergabe von Recallterminen im Vorfeld wurde jedoch nur von einer Minderheit der befragten Gruppe durchgeführt. Eine im Vorfeld festgelegte Recallstruktur kann zum einen sinnvoll sein, da der Patient eine bessere Übersicht über den Behandlungsablauf und die zeitlichen Abstände zwischen den Terminen hat und weniger Gefahr läuft zu viel Zeit zwischen den Behandlungen verstreichen zu lassen und somit den Therapieerfolg zu gefährden. Zum anderen kann seitens des Patienten eine bessere Planung zum Erreichen der Praxis und dem Akquirieren einer Begleitperson erfolgen. Auch aus zahnärztlicher Sicht besteht ein verbesserter Überblick über die erfolgten und geplanten Behandlungen demenzkranker Patienten. Der Nachteil einer im Vorfeld festgelegter Recallstruktur besteht jedoch hauptsächlich in der Nichtinanspruchnahme dieser Termine seitens des Patienten und somit eines Leerlaufes und Verlustgeschäftes im Behandlungsalltag. Hinsichtlich dessen würde eine sinnvolle Lösung in der Vergabe des jeweils auf eine Behandlungssitzung folgenden Termins bestehen, da bei Versäumnis ein geringerer Leerlauf bestünde, aber trotzdem ein ausreichender Überblick über die Behandlungsmaßnahmen des Patienten gewährleistet wäre.

Zusammenfassend lässt sich feststellen, dass die befragten Zahnärzte im Raum Göttingen, Goslar, Holzminen und Northeim hinsichtlich F1.4 nur wenige Aus- und Weiterbildungsmaßnahmen im Bereich der Gerodontologie sowohl auf zahnärztlicher als auch auf personeller Ebene absolviert haben und somit ein großer Verbesserungs- und Weiterbildungsbedarf auf diesem Gebiet besteht. 
Bezüglich des speziellen Praxismanagements zur Betreuung demenzkranker Patienten lassen sich hauptsächlich positive Ergebnisse verzeichnen, vor allem im Rahmen von der zeitlichen und kommunikativen Gestaltung der Patientenbehandlung.

\subsection{Besonderheiten in der zahnmedizinischen Versorgung (F2.1)}

Hinsichtlich der zahnmedizinischen Versorgung demenzkranker Patienten müssen gesonderte Behandlungsmaßnahmen erfolgen. Im Vergleich zur nicht betroffenen Altersgruppe, weisen jene Patienten laut der Fünften Deutschen Mundgesundheitsstudie unbefriedigendere Mundhygienezustände und eine höhere Behandlungsbedürftigkeit auf (Jordan und Micheelis 2016). Neben den intraoralen Verhältnissen müssen im Rahmen der Therapieentscheidung zudem Einflussfaktoren wie Multimorbidität, verminderte Motorik, Sensorik und kognitive Fähigkeit mit einbezogen werden. Grundprinzipien, um das Kauorgan betagter Patienten möglichst lange funktionsfähig zu halten, sind eine leichte Handhabbarkeit, Pflegefähigkeit und Widerstandsfähigkeit des Zahnersatzes (Wöstmann 2003). Auch die Mehrheit der in dieser Studie befragten Zahnärzten versuchen diesen Forderungen gerecht zu werden, indem sie Wert auf ein möglich einfaches und leicht zu handhabendes Prothesendesign legt. Laut Bär et al. (2009) kann hierbei eine frühere Umstellung von festsitzenden auf herausnehmbaren Zahnersatz sinnvoll sein. Im Raum Göttingen, Goslar, Holzminden und Northeim wurde jedoch nur von einer Minderheit der befragten Zahnärzte eine absichtlich verfrühte Umstellung des Zahnersatzes durchgeführt. Grund dafür könnte zum einen der erhöhte Tragekomfort festsitzender Arbeiten, sowie deren bessere Akzeptanz seitens des Patienten sein. Zum anderen könnte die Indikation für einen herausnehmbaren Zahnersatz immer weiter in den Hintergrund rücken, da laut der Fünften Deutschen Mundgesundheitsstudie zunehmend mehr Senioren ihre eigenen Zähne haben (Jordan und Micheelis 2016). Ein weiterer Grund gegen die Entscheidung einer Umstellung des Zahnersatzes kann die geringe Adaptationsfähigkeit dieser Patientengruppe darstellen (Bär et al. 2009), da festsitzender Zahnersatz dem Komfort der natürlichen Bezahnung näher kommt. Diese Situation könnte allerdings umgangen werden, indem man den Patienten schon in einem frühen Stadium der Demenz schrittweise an einen neuen Zahnersatz gewöhnt. Somit könnte eine Empfehlung an die Studienteilnehmer $\mathrm{zu}$ einer möglichst frühen Umstellung auf herausnehmbaren Zahnersatz ausgesprochen werden, da dieser den von Wöstmann (2003) geforderten Grundprinzipien zur Erhaltung des Kauorganes am nächsten kommt.

Um die Pflegefähigkeit des Kauorgans zu vereinfachen und mögliche Infektionsquellen zu beseitigen, könnten prophylaktische Extraktionen fragwürdiger Zähne sinnvoll sein. Zenthöfer et al. (2016) beschreibt den Zusammenhang zwischen mangender Mundgesundheit und persistierender Pathogene im direkten oder indirekten Zusammenhang auf die allgemeine Gesundheit (s. 1.1.1). 
Besonders bei geschwächten, multimorbiden Patienten, kann somit die Beseitigung potentieller Pathogene, wie zum Beispiel durch die Extraktion eines fragwürdigen Zahnes existenziell sein. Auch die Mehrzahl der teilnehmenden Zahnärzte gab an, die Indikation zur Extraktion bei dementen, geriatrischen Patienten strenger zu stellen, möglicherweise begründet auf den bereits genannten Fakten.

Im Rahmen der Fragebogenstudie sollte aufgezeigt werden, ob geriatrischen, dementen Patienten andersartige Mundhygieneartikel empfohlen werden um eine, wenn möglich eigenständige adäquate intraorale Pflege, durchzuführen. Die Mehrheit der befragten Zahnärzte gab an spezielle Mundhygieneartikel zu empfehlen. Aufgrund der motorischen und sensorischen Einschränkungen der Patienten sind andersartige Produkte indiziert und werden in der Literatur umfangreich dargestellt. So sprechen Autoren wie Kluding (2013) Empfehlungen, wie elektrische Zahnbürsten oder Zahnbürsten mit angepassten Griff, zur Vereinfachung der Zahnpflege für Senioren aus. Auch im Rahmen der Auswertung wurden diese genannten Produkte als häufigste Empfehlung für eine für geriatrische Patienten geeignete Zahnbürste angegeben. Im Bereich der Interdentalraumpflege gaben die Studienteilnehmer mit einer großen Mehrheit, die auch in der Literatur empfohlenen Interdentalraumbürsten (Arnetzl und Arnetzl 2013), als besonders geeignet an. Ebenso wurden Mundspüllösungen zur intraoralen Pflege sowohl seitens der befragten Zahnärzte als auch in der Literatur angeraten (Arnetzl und Arnetzl 2013). Zur Prothesenpflege konnte die Reinigung mit Zahnbürsten, sowie mit speziellen Prothesenbürsten und mit Zahnpasta von den Studienteilnehmern als besonders geeignet verzeichnet werden. Auch hier konnten übereinstimmende Empfehlungen gefunden werden (Kluding 2013). Zusammenfassend lässt sich jedoch anmerken, dass in der Literatur, je nach Hersteller verschiedene Empfehlungen für motorisch eingeschränkte, geriatrische Patienten existieren. Eine klare Leitlinie zu speziellen, auf die Bedürfnisse dieser Patientengruppe angepassten Mundhygieneartikeln wurde bisher nicht formuliert. Es ist davon auszugehen, dass die in der Literatur ausgesprochenen Empfehlungen lediglich auf Erfahrungen beruhen und die verschiedenen Möglichkeiten aufzeigen sollen. Durch die Ergebnisse dieser Studie lassen sich ebenso auf Erfahrung beruhende Empfehlungen zur Verbesserung der intraoralen Pflege dementer Patienten formulierten, welche zum Großteil mit der Literatur kongruieren. Letztendlich muss eine an jeden einzelnen Patienten individuell angepasste Mundpflegeinstruktion erfolgen, wobei eine Einbeziehung Dritter im späteren Krankheitsstadium unumgänglich ist. Die, in dieser Studie empfohlenen Mundhygienemittel sollen Hilfestellungen dafür geben, eine auf geriatrische Patienten angepasste intraorale Pflege zu realisieren.

Da demenzkranke Patienten aufgrund ihrer kognitiven Einschränkungen häufig eine geringere Compliance aufweisen, können zur Gebisssanierung Maßnahmen, wie die Behandlung unter Vollnarkose, unumgänglich sein (Bär et al. 2009). Die Minderheit der befragten Zahnärzte gab hierzu an, selbständig eine Behandlung unter Vollnarkose durchzuführen und die Patienten vorwiegend zu 
überweisen. Hierbei sind verschiedene Gründe anzuführen, welche gegen eine Vollnarkose in der Zahnarztpraxis sprechen. Ludwig et al. (2014) beschreiben die mangelnde Infrastruktur zahnärztlicher Praxen, die zur Einhaltung des Sicherheitsstandards bei der Operation und der perioperativen Betreuung demenzkranker, multimorbider Patienten notwendig ist. Während der Behandlung kann eine interdisziplinäre Überwachung des Patienten notwendig werden, wie beispielsweise durch einen Kardiologen, aufgrund der geringeren Belastbarkeit dieser Patientengruppe. Des Weiteren ist eine sorgfältige postoperative Überwachung angezeigt, da mit einem erhöhten Maß an Pflegebedürftigkeit dieser Patienten gerechnet werden muss (Ludwig et al. 2014). Bei der Behandlung geriatrischer Patienten unter Vollnarkose muss außerdem eine strenge Abwägung zwischen Risiken und Nutzen seitens des Zahnarztes gestellt werden. Als mögliche Komplikation kann es zu einer Postoperativen kognitiven Dysfunktion (POCD) kommen, welche in unmittelbaren Zusammenhang mit dem Alter der Patienten steht und zu einer Verschlechterung des neuropsychiatrischen Zustandes führt (Kratz et al. 2005). Aus diesen Gründen könnte nachvollzogen werden, weshalb nur eine geringe Menge der befragten Zahnärzteschaft eine eigenständige Behandlung demenzkranker Patienten unter Vollnarkose durchführt. Um dennoch eine adäquate zahnärztliche Therapie bei Patienten mit geringer Compliance zu realisieren, wurde im Rahmen der Studie nach dem Gebrauch von hierbei zum Einsatz kommenden sedierenden Medikamenten gefragt. Die Mehrheit der Studienteilnehmer gab an, keine Sedativa bei dieser Patientengruppe zu verwenden. Als zum Einsatz kommende Medikamente wurden im Freitext Dormicum (Midazolam), Lachgas (Distickstoffmonoxid) und Diazepam ergänzt. Anzuführen ist hierbei, dass es auch bei den sedierenden Medikamenten, wie den angeführten Benzodiazepinen (Diazepam, Midazolam), zu einer Begünstigung einer POCD kommen kann (Sinner 2009). Hierbei ist zu ergänzen, dass das Restrisiko einer postoperativen kognitiven Störung durch den Gebrauch eines kurzwirksamen Medikament dieser Gruppe (z. B. Dormicum) anstelle eines langwirksamen (z. B. Diazepam) reduziert werden kann (Rundshagen 2014).

Aus dem Grund der unsicheren postoperativen Diagnose ließe sich erklären, weshalb die befragte Zahnärzteschaft mit einer großen Mehrheit von Sedativa zur Behandlung von Demenzpatienten Abstand nimmt. Das als weiteres genannte, zum Einsatz kommende sedierende Medikament Lachgas muss in diesem Zusammenhang separat betrachtet werden. Mathers (2015) beschreibt hierbei die anxiolytische Wirkung des Gases als hilfreich und bedenkenlos einsetzbar bei der dentalen Sedierung dementer Patienten. Lachgas reduziere durch den abfallenden Stress nicht nur die kardiovaskulären Folgen, sondern könne gleichzeitig die Sauerstoffversorgung verbessern und somit die Kooperationsbereitschaft während der Behandlung optimierten (Mathers 2015). Jedoch ist in diesem Zusammenhang anzumerken, dass es unter bestimmten Umständen zu Gesundheitsrisiken bei der Verwendung von Lachgas kommen kann. Aufgrund der raschen Diffusion des Gases in luftgefüllte Körperhohlräume, kann es zu einer Druckerhöhung in diesen kommen. Dementsprechend muss bei 
Patienten mit unter anderem einem Pneumothorax, Pneumencephalon, Ileus, einer Luftembolie oder Trommelfelleingriffen, von einer Lachgassedierung Abstand genommen werden. Weitere Kontraindikationen sind Atemwegsveränderungen, Vitamin B 12-Mangel oder Herzerkrankungen. Als mögliche Nebenwirkungen kann es zu Übelkeit, Erbrechen, Schwindel oder Angstzuständen kommen (Liakoni und Liechti 2015). Es ist anzumerken, dass der Einsatz von Lachgas im Bereich der Behandlungsoptimierung von Patienten mit geringer Compliance im befragten Gebiet mehr etabliert werden kann, um eine adäquate zahnmedizinische Therapie zu realisieren. Eine stattdessen erfolgende Vollnarkose oder Behandlung mit Benzodiazepinen mit ihren Risiken, könnte bei Erfolg somit reduziert oder komplett umgangen werden. Als Voraussetzung gilt in diesem Zusammenhang jedoch eine unauffällige Anamnese des Patienten bezüglich der möglichen Behandlungsrisiken beim Einsatz von Lachgas.

Bezüglich der verschiedenen Praxisformen ließ sich anhand der Auswertung feststellen, dass eine Behandlung unter Vollnarkose oder der Einsatz von sedierenden Medikamenten häufiger in Gemeinschaftspraxen durchgeführt werden. Möglicherweise lässt sich dies damit erklären, dass innerhalb einer Gemeinschaftspraxis breitgefächerte Spezialisierungen unter den einzelnen Behandlern vorliegen und somit häufiger Fachpersonal für die Durchführung dieser Behandlungen vorhanden ist. Da außerdem ein erhöhter Personalaufwand benötigt wird, kann vermutet werden, dass in Gemeinschaftspraxen hinsichtlich dieses Umstandes bessere Voraussetzungen gegeben sind. Des Weiteren ist anzuführen, dass die Vergütung von Narkosen im zahnärztlichen Bereich nicht aufwandsgerecht kalkuliert sind und somit einen erhöhten Kostenpunkt für den Zahnarzt darstellen würde (Ludwig et al. 2014). Gemeinschaftspraxen könnten sich somit aufgrund der gemeinsamen Kostenlast und der steuerlichen Vorteile (Nuri 2015) häufiger dazu entschließen trotz der anfallenden Kostenpunkte eine solche Behandlung durchzuführen.

Zusammenfassend lässt sich bezüglich der Fragestellung 2.1 feststellen, dass es in der Versorgung von demenzkranken Patienten Besonderheiten in Bezug auf die nicht betroffene Altersgruppe gibt. Dies zeigt sich unter anderem bei der Zahnersatzgestaltung und der Empfehlung von Mundhygieneartikeln. Hier konnten häufig Parallelen zur Literatur gezogen und somit davon ausgegangen werden, dass die befragten Zahnärzte im Raum Göttingen, Goslar, Northeim und Holzminden mit großer Mehrheit nach den allgemein vertretenden Empfehlungen und Prinzipien zur Behandlung dieser Patientengruppe, vorgehen. Hinsichtlich der Behandlungsweise demenzkranker Patienten mit geringer Compliance ist festzustellen, dass die befragten Zahnärzte sehr kalkuliert mit sedierenden und narkotisierenden Maßnahmen umgehen. Dies ist zum einen positiv zu bewerten, da mögliche Risiken einer POCD und weiteren postoperativen Folgen somit umgangen werden können. Zum anderen könnte der Einsatz vom weniger schädlichem Lachgas, bei entsprechend positiven körperlichen Voraussetzungen des Patienten, in diesem Zusammenhang gesteigert werden, um einer möglichen, daraus resultierenden intraoralen Unterversorgung entgegenzuwirken. 


\subsection{Intraorale Besonderheiten bei demenzkranken Patienten (F2.2)}

Mit Hilfe der Fragebogenstudie soll aufgezeigt werden, ob demenzkranke Patienten im Raum Göttingen, Goslar, Holzminden und Northeim im Vergleich zur nicht betroffenen Altersgruppe häufiger intraorale Auffälligkeiten vorweisen. Im Rahmen der Fünften Deutschen Mundgesundheitsstudie konnte bereits im deutschlandweiten Vergleich aufgezeigt werden, dass pflegebedürftige Patienten einen schlechteren Mundhygienestatus aufweisen als nicht erkrankte, gleichaltrige Patienten (Jordan und Micheelis 2016). Die Zahnärzte im befragten Gebiet gaben diesbezüglich an, tendenziell ebenfalls einen schlechteren Mundhygienestatus bei dieser Patientengruppe zu verzeichnen und daraus resultierende intraorale Auffälligkeiten feststellen zu können. Auch ähnliche Studien, wie die SÄVIP-Studie (Hallauer et al. 2005) und die Studie von Besimo und Besimo-Meyer (2015) zur oralen Gesundheit von Menschen mit Demenz, legen die intraorale Unterversorgung der betroffenen Zielgruppe offen und bestätigen die auch im befragten Raum geäußerten Tendenzen im internationalen Vergleich.

Die am häufigsten angegebenen Einschätzungen spezifischer Auffälligkeiten demenzkranker Patienten waren Karies, Gingivitis und Parodontitis. Bezüglich der erhöhten Kariesprävalenz dieser Patientengruppe konnten in der Literatur Parallelen gefunden werden, welche die Einschätzung der befragten Zahnärzte bestätigen. Reißmann und Lamprecht (2017) gaben hierzu an, dass Patienten mit einer schweren psychischen Erkrankung, wie Demenz eine wesentlich schlechtere Mundgesundheit und ein höheres Kariesrisiko aufweisen. Auch Baumgartner et al. (2015) beschrieben in diesem Zusammenhang die höhere Kariesprävalenz demenzkranker Patienten sowie zusätzlich das erhöhte Auftreten von Parodontopathien als bei gesunden Gleichaltrigen. Da einer Parodontitis immer eine Gingivitis vorausgeht, ist davon auszugehen, dass, wie auch von den Studienteilnehmern angegeben, eine erhöhte Gingivitisprävalenz bei demenzkranken Patienten vorliegt. Eine häufigere Wahrscheinlichkeit zum Auftreten einer Xerostomie konnte von den Studienteilnehmern nicht eindeutig identifiziert werden und wurde im Rahmen der Fragebogenstudie mit der Antwort „unentschlossen“ beurteilt. Autoren wie Baumgartner et al. (2015) beschrieben jedoch in diesem Zusammenhang die Beobachtung von einer erhöhten Xerostomieprävalenz dieser Patientengruppe. Ursache für die häufig auftretende Mundtrockenheit stellt hierbei die Nebenwirkung der mit der Multimorbidität einhergehenden Polypharmazie dar (Baumgartner et al. 2015). Der Zusammenhang einer reduzierten Speichelrate und einer damit einhergehenden erhöhten Plaqueanlagerungen (s. 1.1.2) würde zusätzlich die vermehrte Entstehung von Karies und Parodontopathien demenzkranker Patienten erklären (s. Abbildung 16). 


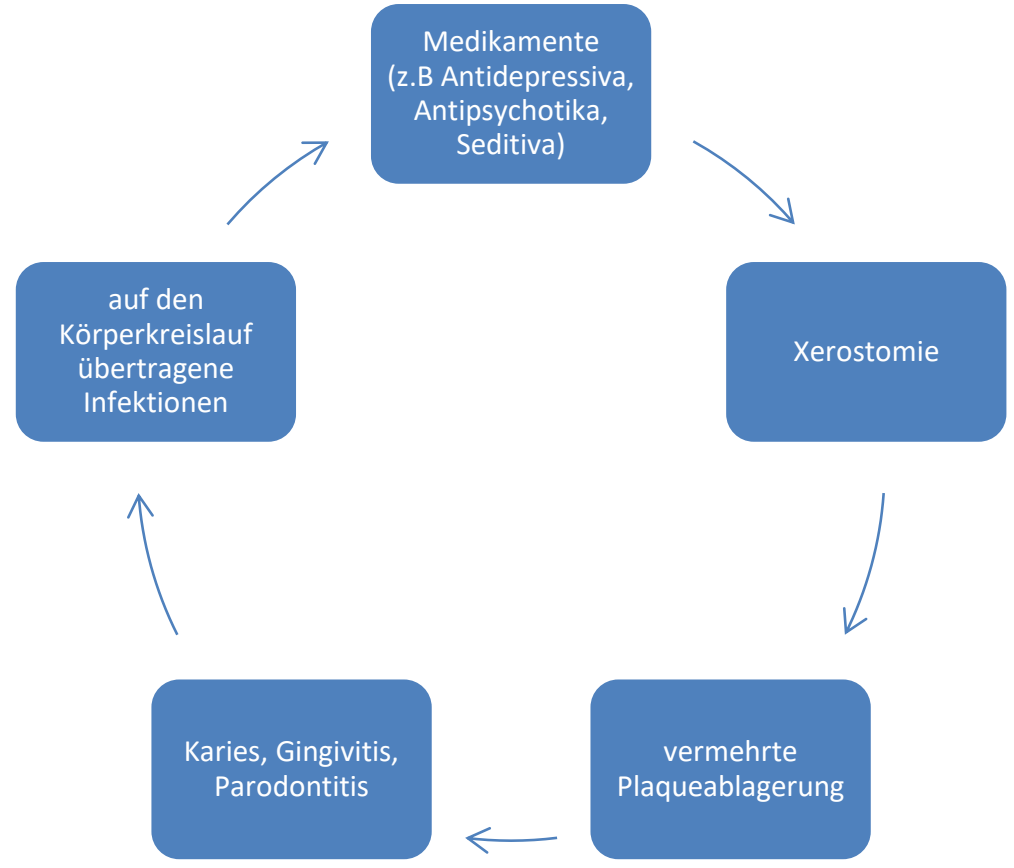

Abbildung 16 Zusammenhang zwischen Polypharmazie und intraoralen Erkrankungen (basierend auf Baumgartner et al. 2015)

Als nicht vermehrt auftretende intraorale Auffälligkeiten im Vergleich zu nicht betroffenen Altersgruppe wurden von den Studienteilnehmern kanzerogene Veränderungen und Hypersalivation der Mundhöhle angegeben. Bezüglich des vermehrten Speichelflusses ist der Literatur zu entnehmen, dass Patienten mit einer Parkinsondemenz aufgrund der medikamentösen Therapie eine höhere Prävalenz zur Hypersalivation aufweisen als die nicht erkrankte Altersgruppe (Grossmann und Pirker 2004). Da diese Form der Demenz deutlich weniger verbreitet ist als die üblich in der Zahnarztpraxis anzutreffenden Formen, wie die Alzheimer-Demenz und die vaskuläre Demenz, ist davon auszugehen, dass die Studienteilnehmer aufgrund der geringen Patientenzahl keinen Zusammenhang zwischen Hypersalivation und demenzkranken Patienten herstellen konnten. Hinsichtlich eines möglichen vermehrten Auftretens von kanzerogenen Veränderungen bei demenzkranken Patienten, konnte von den Studienteilnehmern keine erhöhte Prävalenz festgestellt werden. In der Literatur konnten ebenfalls keine eindeutigen Zusammenhänge gefunden werden, dass eine vorliegende Demenz das Krebsrisiko in der Mundhöhle fördert. Allerdings ist darauf hinzuweisen, dass mit ansteigendem Lebensalter die Prävalenz kanzerogener Veränderungen steigt. So liegt der Altersgipfel bei Plattenepithelkarzinomen im sechsten Lebensjahrzehnt. Dies lässt vermuten, dass in der von Demenz hauptsächlich betroffenen Altersgruppe eine erhöhte Krebsrate in der Mundhöhle verzeichnet werden kann (Maier et al. 1990). Wie der SÄVIP-Studie (Hallauer et al. 2005) und der Fünften Deutschen Mundgesundheitsstudie (Jordan und Micheelis 2016) entnommen werden kann, liegt im Bereich der pflegebedürftigen Senioren eine intraorale Unterversorgung vor. Diese Unterversorgung bezieht sich unter anderen auch auf die Qualität des Zahnersatzes. Wenn für den 
kognitiv eingeschränkten Patienten keine Möglichkeit besteht, die pflegende Person auf Druckstellen und schmerzende Prothesenbereiche hinzuweisen, besteht die Gefahr einer dauerhaften mechanischen Überreizung des intraoralen Gewebes. Solche Überreizungen stellen ein Risikofaktor für die Entstehung von kanzerogenen Veränderungen dar (Gumpp und Henß 2014). Neben mechanischen Faktoren ist eine schlechte Mundhygiene, welche ebenfalls im Bereich der geriatrischen, pflegebedürftigen Patienten durch die angeführten Studien dargelegt wurde, ein Risikofaktor zur Entstehung intraoraler Tumore (Gumpp und Henß 2014).

Zusammenfassend lässt sich feststellen, dass in der Gruppe der geriatrischen Patienten durch verschiedene beeinflussende Faktoren ein erhöhtes Risiko besteht an intraoralen Karzinomen zu erkranken. Im Rahmen dieser Arbeit sind lediglich Zusammenhänge angeführt, die auf ein vermehrtes Auftreten schließen lassen. Jedoch konnte keine speziell für demenzkranke Patienten erhöhte Prävalenz im Rahmen der Literaturrecherche, sowie durch die Auswertung der Ergebnisse der Fragebogenstudie festgestellt werden.

\subsection{Beobachtung demenzkranker Patienten in der zahnmedizinischen Praxis (F3)}

Besimo und Besimo-Meyer (2016) empfehlen eine oralmedizinische Langzeitbetreuung, um die sich ändernden Ressourcen von kognitiv eingeschränkten Patienten zu überprüfen, damit rechtzeitig präventive und therapeutische Maßnahmen angepasst werden können. Um diese Ressourcen einzuschätzen, sollte im Rahmen der Studie herausgefunden werden, ob Testverfahren oder

Screeningbögen zur Anwendung kommen oder weitere Besonderheiten in der Beobachtung demenzkranker Patienten zu verzeichnen sind. Die Mehrheit der Studienteilnehmer gab hierbei an, dass diese Patientengruppe keiner besonderen Beobachtung in der Praxis unterliegt. Besimo (2009) beschreibt in diesem Zusammenhang die Wichtigkeit eines medizinisch-psychosozialen Screenings geriatrischer Patienten. Mit dem frühzeitigen Erkennen von intraoraler Erkrankungen können mögliche Wechselwirkungen zwischen oralen und systemischen Erkrankungen umgangen werden und somit eine Verschlechterung der gesundheitlichen sowie psychosozialen Situation des multimorbiden Patienten, wie auch Komplikationen bei der zahnärztlichen oder allgemeinärztlichen Therapie verhindert werden (Besimo 2009). Um diese Umstände zu umgehen, dient das Screeningverfahren zur Einschätzung der Bedürfnisse des demenzkranken Patienten um eine frühzeitige Anpassung des Zahnersatzes und der intraoralen Versorgung zu gewährleisten und eine etwaige Unter- oder Über- sowie Fehlversorgung zu vermeiden. Dementsprechend können Empfehlungen ausgesprochen werden, in jeder zahnmedizinischen Praxis Testverfahren zur Überprüfung der kognitiven Ressourcen anzuwenden um eine medizinische interdisziplinäre Zusammenarbeit zu gewährleisten und den Patienten hinsichtlich der Erkrankung adäquat zu 
versorgen. Eine Überprüfung der kognitiven Leistungen in der Zahnarztpraxis ist zudem generell sinnvoll, da der Großteil der Patienten zu den halbjährigen Prophylaxeterminen vorstellig wird und somit eine regelmäßige Beobachtung erfolgen kann. Glenz et al. (2015) beschreibt ein solches Screeningverfahren in diesem Zusammenhang als mit nur sehr geringen zeitlichen und finanziellen Mehraufwand routinemäßig in der Praxis einsetzbar.

Durch die kognitive Einschränkung ist der demenzkranke Patient häufig nicht mehr in der Lage, eigenständige Entscheidungen zu treffen. Aus diesem Grund ist die Anwesenheit eines gesetzlichen Betreuers für die Gesundheits- und Vermögenssorge bei nicht einwilligungsfähigen Patienten juristisch vorgesehen (Ludwig 2016). Im Rahmen der Studie wurde die Anwesenheit Dritter während der zahnmedizinischen Behandlung mit einer Wahrscheinlichkeit von „häufig“ bis „immer“ eingeschätzt. Dementsprechend lässt sich schlussfolgern, dass Behandlungen demenzkranker Patienten in rechtlicher Hinsicht in seltenen Fällen nicht ordnungsgemäß durchgeführt wurden. Zu vermuten ist, dass befragte Zahnärzte auch Einschätzungen zu geriatrischen, nicht eindeutig als demenzkrank diagnostizierten Patienten oder Erkrankten in einem anfänglichen Stadium im Rahmen dieser Frage angegeben haben. Zusammenfassend ist eine klare Empfehlung für die befragten Praxen zur genauen Beobachtung und Beurteilung demenzkranker Patienten auszusprechen, um die Rechtslage während der Behandlung nicht zu gefährden.

Im Rahmen der Auswertung der Forschungsfragestellung F3, konnten signifikante Unterschiede zwischen dem Geschlecht der Behandler und zwischen den Praxisformen festgestellt werden. Somit unterlagen demenzkranke Patienten häufiger einer besonderen Beobachtung von weiblichen Behandlern als von männlichen. Um diesen Umstand zu begründen, können zunächst nur Vermutungen angeführt werden, da wissenschaftliche Studien zu dieser Thematik fehlen. Dem Pflegereport 2015 kann in diesem Zusammenhang entnommen werden, dass neun von zehn Personen in der häuslichen Pflege Frauen sind (DAK 2015) und diese Thematik somit aus weiblicher Sicht größeren Bestand haben könnte als aus männlicher. Des Weiteren konnte verzeichnet werden, dass signifikant häufiger demenzkranke Patienten einer besonderen Beobachtung in Gemeinschaftspraxen unterlagen als in alleinigen. Grund könnte das größere Praxisteam in Gemeinschaftspraxen sein, da mehr Personal und Behandler vorhanden sind um diesbezüglich Beobachtungen anzustellen. Wissenschaftliche Studien dahingehend sind jedoch ebenfalls nicht existent.

Zusammenfassend lässt sich feststellen, dass insgesamt nur selten eine besondere Erfassung demenzkranker Patienten zu verzeichnen ist, und es diesbezüglich im befragten Raum ein großer Verbesserungsbedarf besteht. 


\subsection{Diskussion zusätzlicher Fragen}

Im Rahmen der Fragebogenstudie sollte abschließend aufgezeigt werden, welcher Verbesserungsbedarf im Umgang mit dementen Patienten seitens der Zahnärzte besteht. Als am häufigsten verbesserungsbedürftig wurde die mangelnde Schulung des Pflegepersonals im Altersheim angesehen. Studien belegen in diesem Zusammenhang, dass dem Pflegepersonal theoretisches Wissen über Zahn- und Mundgesundheit, sowie Mundhygienemittel und der zeitliche Rahmen fehlt, um eine adäquate intraorale Pflege auszuführen, sowie Pathologien zu erkennen (Marmy und Matt 2003; Baumgartner et al. 2015). Bereits in der bundeseinheitlichen Altenpflegeausbildung besteht keine ausreichende Verankerung der Zahn-, Mund- und Prothesenpflege mit nur ca. sechs Stunden in drei Jahren Ausbildung (Ludwig 2009). Mehrere Studien aus der internationalen Literatur gaben darüber hinaus an, dass in Pflegeheimen oft noch nie eine Fortbildung zur Mundhygiene durchgeführt wurde (Jäger et al. 2009). Neben diesen Rückständen in der Aus- und Weiterbildung des Pflegepersonals beschreiben Jäger et al. (2009), dass Pflegemaßnahmen im Mund der Heimbewohner als eine besonders unangenehme Tätigkeit von Seiten des Pflegepersonals dargestellt werden. Intraorale Pflege werde dementsprechend seltener durchgeführt als die Reinigung einer Prothese außerhalb der Mundhöhle (Jäger et al. 2009). Mangelnde theoretische Grundkenntnisse sowie psychologische Barrieren bei der praktischen Durchführung, führen somit zu einer inadäquaten Mundhygiene und intraoralen Unterversorgung der Heimbewohner. Ludwig (2009) fordert in diesem Zusammenhang eine nachhaltige Verbesserung der Pflegekompetenz im Rahmen von Ausbildungs- wie auch Fortbildungsangeboten in der Zahn-, Mund- und Zahnersatzpflege auf theoretischer und praktischer Ebene. Diverse in Deutschland durchgeführte Studien und Projekte, wie z. B. „Altern mit Biss“ in Sachsen-Anhalt oder „Teamwerk“ in München, belegen, dass eine umfangreiche Schulung des Pflegepersonals zu einer nachhaltigen Verbesserung der intraoralen Situation der Heimbewohner führt (Krömer 2018). Die mangelnde Schulung des Pflegepersonals, kann somit als einer der wichtigsten Gründe für die intraorale Unterversorgung geriatrischer Patienten in pflegenden Institutionen angesehen werden. Die aus der unzureichenden Mundhygiene resultierenden gesundheitlichen Folgen, sind unter 1.1.1 erläutert und explizieren die Wichtigkeit einer Verbesserung in der Aus- und Weiterbildung der Pflegekräfte.

Um die zahnmedizinische Situation demenzkranker Patienten zu bessern, gaben die befragten Zahnärzte im Rahmen der Studie an, dass eine höhere finanzielle Unterstützung zur Behandlung dieser Patientenklientel in Verbindung mit dem erforderten Mehraufwand, vorliegen muss. Diese Problematik wurde seitens der Kassenzahnärztlichen Bundesvereinigung und der Bundeszahnärztekammer bereits erkannt, sodass eine stetige Verbesserung der finanziellen Unterstützung in Form von steigenden Honoraren und zusätzlichen Zuschlagspositionen generiert werden konnte (s. 1.1.2). Würde eine aufwandsgerechte finanzielle Unterstützung existieren, wäre davon auszugehen, dass die zahnmedizinische Versorgungssituation eine deutliche Verbesserung 
erfährt und die Häufigkeit der intraoralen Unterversorgung bei demenzkranken Patienten sinken würde.

Zusammenfassend lässt sich feststellen, dass die inadäquate Mundhygienesituation laut der Studienteilnehmer zum größten Teil auf die mangelnde zahnmedizinische Ausbildung des Pflegepersonals, sowie auf die fehlende finanzielle Unterstützung zur Behandlung demenzkranker Patienten zurückzuführen ist. Auch die Literatur bestätigt Rückstände auf diesen Gebieten, welche somit als wichtige Angriffspunkte zur Verbesserung der zahnmedizinischen Situation Demenzkranker angesehen werden können.

\subsection{Limitationen}

In der vorliegenden Arbeit wurden die Daten mit Hilfe einer schriftlich-postalischen Befragung der Zahnärzteschaft erfasst. Diese Form der Befragung stellte sich mit einer Rücklaufquote von 29,31\% als günstig heraus. Insgesamt wurden von den 406 versendeten Fragebögen 119 beantwortet zurückgesendet. Laut Diekmann (1995) erzielen Rücklaufquoten bei der schriftlichen Befragung selten über $20 \%$ und somit unter dem in dieser Studie generierten Datensatz. Um einen hohen Rücklauf zu erhalten, wurde jedem Fragebogen ein umfassendes Anschreiben beigefügt. Zum einen erhielten die Teilnehmer dadurch Aufschluss über die vorliegende Studie durch eine Kurzfassung des Projektes, zum anderen wurden sie über die rechtlichen und ethischen Aspekte, sowie den Datenschutz aufgeklärt. Diesem Begleitschreiben kommt nach Barth (1998) eine sehr große Bedeutung zu, da die Teilnehmer mit plausiblen Argumenten zur Teilnahme angeregt werden und eventuell bestehendes Misstrauen beseitigt werden kann. Um an möglichst viele Studienteilnehmer zu appellieren, verfasste Barth folgende Prinzipien: zum einen solle eine Adresse oder Telefonnummer für eventuelle Rückfragen hinterlegt sein, in einem geringen Umfang müsse über das Projekt und dessen Wichtigkeit aufgeklärt und außerdem die Anonymität und der wissenschaftliche Charakter der Studie betont werden, sowie abschließend auf einen konkreten Rücklauftermin hingewiesen werden (Barth 1998). Da das im Rahmen dieser Studie beigefügte Begleitschreiben alle beschriebenen Kriterien erfüllte, kann davon ausgegangen werden, dass es dazu beigetragen hat, eine erhöhte Rücklaufquote zu generieren. Neben dem Begleitschreiben wurde dem Fragebogen außerdem ein frankierter Rückumschlag beigefügt, welcher es den Teilnehmern erleichtern sollte, die beantworteten Bögen zurückzusenden und dementsprechend zu einer Rücklaufsteigerung beitrug. Schlussfolgernd lässt sich sagen, dass sich die Variante der schriftlichpostalen Befragung mit beigefügten Begleitschreiben und frankierten Rückumschlag, hinsichtlich der Rücklaufquote von 29,31\%, als günstige Methode zur Datengenerierung darstellte. Als eine weitere mögliche Variante zur Erfassung der Daten kam die Online-Befragung über E-Mail in Betracht. Ein möglicher Vorteil könnte die bessere Erreichbarkeit der Zielgruppe sein, da der 
Fragebogen auf verschiedenen Servern der Praxis zur Verfügung stünde oder gegebenenfalls auch über das Smartphone beantwortet werden könnte. Auch wäre die Bereitstellung frankierter Rückumschläge hinfällig, was zum einen in finanzieller als zum anderen auch in praktischer Hinsicht einen Vorteil darstellen könnte. Durch die Online-Variante hätte gegebenenfalls eine höhere Rücklaufquote generiert werden können, da eine orts- und zeitunabhängige Beantwortung der Fragen möglich gewesen wäre. Dieses Modell konnte für die vorliegende Arbeit jedoch nicht in Betracht gezogen werden, da nur wenige E-Mail-Adressen online bei der Zahnärztekammer Niedersachen hinterlegt waren und keine Möglichkeit bestand, die Fragebögen über einen E-Mail-Verteiler der Kammer zu senden. Somit wäre die Zielgruppe deutlich kleiner ausgefallen, als bei der schriftlichpostalen Befragung.

Hinsichtlich der Gestaltung der Fragebögen rät Barth (1998), dass diese nicht zu viele Seiten umfassen dürfen, da sonst die Bereitschaft zum Ausfüllen sinke. Des Weiteren würden sich geschlossene Fragen mehr eignen, da für offene Fragen die Antwortbereitschaft höher sein muss und sie sich somit negativ auf den Gesamtrücklauf auswirken kann und außerdem im Rahmen der Vergleichbarkeit Probleme darstellt. Der in dieser Arbeit vorliegende Fragebogen entspricht zum größten Teil den angeforderten Gesichtspunkten. Mit fünf Seiten stellt er einen akzeptablen Umfang für die Studienteilnehmer dar. Des Weiteren wurden mehrheitlich geschlossene Fragen gestellt, welche eine geringere Antwortbereitschaft als offene Fragen fordern und somit zu einer vergrößerten Datenmenge beitrugen. Es war festzustellen, dass wie in der Literatur beschrieben, bei einigen offenen Fragen eine geringe Bereitschaft zur Beantwortung vorhanden war. Zusammenfassend ließ sich feststellen, dass anhand des vorliegenden Fragebogens alle Schwerpunkte, welche unter 1.5 in den Forschungsfragestellungen formuliert wurden, ausreichend durch die Teilnehmer beantwortet werden konnten. Im Rahmen der Auswertung stellten sich jedoch einige offene, zu ergänzende Antwortmöglichkeiten als nicht günstig heraus, da sie durch die Teilnehmer in unzureichender Häufigkeit beantwortet wurden. Dies lässt zum einen vermuten, dass entweder die Fragestellung ungünstig getroffen wurde oder dass die Teilnehmer sich nicht ausreichend mit dem Fragebogen und insbesondere mit der Thematik befassten, da es nur wenige Ergänzungen zu den bereits aufgelisteten Besonderheiten im Umgang mit demenzkranken Patienten im Praxisalltag gab.

Die Studienpopulation von 406 Teilnehmern aus den Räumen Göttingen, Holzminden, Goslar und Northeim wurde anhand der online aufgelisteten Zahnärzte der Zahnärztekammer Niedersachsen generiert. Dieser Weg über die Kammer erwies sich zur Erfassung der Studienteilnehmer als günstig, da in kurzer Zeit alle Adressen vollständig zusammengetragen werden konnten. Allerdings muss darauf hingewiesen werden, dass die Aufnahme in die Datenbank das Einverständnis der Zahnärzte erfordert und somit keinen Anspruch auf Vollständigkeit erhebt ${ }^{6}$. Somit konnte mit der vorliegenden

\footnotetext{
${ }^{6}$ https://zkn.de/patienten/zahnarztsuche-notfall.html, Zugriff am 04.03.18
} 
Studie nur ein Teil der Ärzteschaft der besagten Räume erreicht werden, sodass die Ergebnisse eher als richtungsweisend interpretiert werden können.

\subsection{Ausblick}

Anhand der vorliegenden Ergebnisse der deskriptiven Fragebogenstudie sollten mögliche Verbesserungsmaßnahmen im Umgang mit, und der zahnmedizinischen Versorgung von demenzkranken Patienten, sowie zukünftige weiterführende Studien und Überlegungen zu dieser Thematik aufgezeigt werden. Festzustellen ist, dass aus zahnärztlicher Sicht in den Räumen Göttingen, Goslar, Holzminden und Northeim eine überwiegend günstige zahnmedizinische Versorgungssituation für demenzkranke Patienten besteht. Sowohl der Zugang zur zahnärztlichen Behandlung in der Praxis als auch die Versorgung im Altersheim und in häuslichen Settings stellen sich im Vergleich mit nationalen und internationalen Studien als vorwiegend gesichert heraus. In diesem Zusammenhang ist jedoch zu bemerken, dass die Einschätzung lediglich auf zahnärztlichem Aspekt beruht und die Sichtweise der Patienten, sowie deren Betreuer nicht mit einbezogen wurde. Um eine umfassende Aussage zur zahnmedizinischen Betreuungs- und Versorgungssituation der befragten Gebiete zu treffen, müssen zukünftige Studien erfolgen, welche sowohl den Blickwinkel der Patienten, bzw. deren gesetzlichen Vormund, sowie den der ansässigen Institutionen, wie Altersheime und Pflegedienste, berücksichtigen.

Zur Verbesserung der zahnmedizinischen Situation konnten anhand der Studie wichtige Aspekte für mögliche Rückstände in der Betreuung und Versorgung demenzkranker Patienten aufgezeigt werden. Diese beziehen sich zum einen auf die nur geringfügig in Anspruch genommenen Weiterbildungsmaßnahmen seitens der Zahnärzteschaft und des Personals auf dem Gebiet der Gerodontologie. Ebenfalls anzuführen ist in diesem Zusammenhang die unter 4.4 beschriebene mangelnde Ausbildungssituation im Rahmen des zahnmedizinischen Studiums. Zusammenfassend ist somit die Aus- und Weiterbildungssituation auf dem Gebiet der Gerodontologie stark verbesserungsfähig, insbesondere aufgrund des demografischen Wandels und der damit zunehmenden Relevanz dieses Themas. Als weiterer beeinflussender Aspekt, lässt sich die finanzielle Unterstützung, in Form der kassenzahnärtzlichen Honorierung im Bereich der Seniorenzahnmedizin und dem verbundenen Mehraufwand, anführen. Würde die Rentabilität der Arbeit auf dem Gebiet der Gerodontologie verbessert werden, wäre es möglich, dass zunehmend Spezialisierungen seitens der Zahnärzteschaft erfolgen und die Rückstände auf dem Gebiet der Weiterbildungen reduziert werden können. Zusätzlich muss im Zusammenhang der Aus- und Weiterbildungssituation die vorwiegend mangelnden Qualifikationen der Pflegekräfte im Bereich der Zahnheilkunde als weiteres Problem angesehen werden (s. 4.8). Zukünftig müsste sich die Situation seitens des Pflegepersonals sowohl hinsichtlich zeitlicher als auch fachlicher Aspekte zur 
Durchführung einer adäquaten intraoralen Beurteilung und Pflege verbessern, um der bestehenden intraoralen Unterversorgung geriatrischer Patienten entgegenzuwirken.

Zusammenfassend steht im Bereich der Gerodontologie die interdisziplinäre Zusammenarbeit zwischen den ansässigen Institutionen, wie Altersheimen, Pflegediensten, sowie gesetzlichen Betreuern und dem Zahnarzt im Vordergrund. Um eine adäquate Patientenversorgung zu gewährleisten, müssen alle beteiligten Disziplinen umfassend fachgerecht geschult und ausgebildet sein, um Missstände aufzudecken und beseitigen zu können. Beeinflussende Faktoren, wie zeitliche oder finanzielle Umstände, müssen in diesem Zusammenhang ausreichend berücksichtigt werden, um eine zukünftig reibungslose Versorgung zu gewährleisten. 


\section{Zusammenfassung}

In der vorliegenden Arbeit sollte mittels einer deskriptiven Fragebogenstudie aufgezeigt werden, wie demenzkranke Patienten im Raum Göttingen, Goslar, Holzminden und Northeim zahnmedizinisch versorgt und behandelt werden und welcher Verbesserungsbedarf in diesem Zusammenhang besteht. Im ersten Teil wurden allgemeine Fakten zur zahnmedizinischen Versorgungssituation demenzkranker Patienten mittels Literaturrecherche zusammengetragen, um bereits vorhandene Rückstände aufzuzeigen und die Wichtigkeit einer adäquaten intraoralen Versorgung dieser Patienten zu verdeutlichen. Inwiefern dieser Bedarf in der Zahnmedizin Bestand hat und stetig weiterwächst, lässt sich anhand des demografischen Wandels in Deutschland erschließen.

Insgesamt konnten durch die postalische Befragung die Daten von 119 Zahnärzten, 31,1\% davon Frauen und 68,9 \% Männer, generiert werden. Es wurden verschiedene Forschungsfragestellungen formuliert, um die unterschiedlichen Aspekte der zahnmedizinischen Betreuung und Versorgung demenzkranker Patienten zu betrachten, um Missstände aufzudecken, sowie Verbesserungsmaßnahmen abzuleiten. Diese Teilaspekte zielten auf (1) die Konsultation und Erreichbarkeit der Praxis für demenzkranke Patienten, die Praxisausstattung, Fort- und Weiterbildungsmaßnahmen seitens der Zahnärzteschaft, (2) Besonderheiten in der Versorgung und Behandlung, sowie (3) die besondere Beobachtung demenzkranker Patienten ab.

Hinsichtlich der Auswertung der Fragebögen lässt sich für demenzkranke Patienten aus Sicht der Zahnärzteschaft im befragten Raum eine überwiegend günstige Versorgungssituation beschreiben. Die Praxisausstattung, deren Erreichbarkeit, der Umgang und die Versorgung demenzkranker Patienten können zum größten Teil mit den allgemeinen Empfehlungen und Prinzipien aus der Literatur und vergleichbaren Studien in Einklang gebracht werden. Rückstände ließen sich unter anderem auf dem Gebiet der Fort- und Weiterbildungsmaßnahmen seitens der Zahnärzte, sowie des Praxispersonals, sowie bezüglich der besonderen Beobachtung demenzkranker Patienten, speziell eingesetzter Screeningverfahren, verzeichnen. Auch im Rahmen der Auswertung besonderer Behandlungsmaßnahmen und der zum Einsatz kommenden Mundhygieneprodukten konnten mit Hilfe der Literaturrecherche weitere Ergänzungen vorgenommen werden, welche zusätzlich als Empfehlungen dienen sollen, eine auf den geriatrischen Patienten angepasste intraorale Versorgung zu realisieren. Des Weiteren wurden Verbesserungsvorschläge seitens der Zahnärzteschaft dargestellt und diskutiert, um die zahnmedizinische Betreuungs- und Versorgungssituation im besagten Gebiet umfangreich zu bewerten.

Anhand der ausgewerteten Ergebnisse muss angemerkt werden, dass diese Studie nur eine eingeschränkte Auskunft zur tatsächlichen zahnmedizinischen Versorgungssituation demenzkranker Patienten im befragten Gebiet geben kann. Die Einschätzungen beruhen lediglich auf der Sichtweise der Zahnärzte und vernachlässigt die Aspekte der ansässigen Pflegeinstitutionen und der Patienten. 
Des Weiteren konnten mit Hilfe der online aufgelisteten Zahnärzte der Zahnärztekammer Niedersachsen nur ein eingeschränkter Zugriff auf die Adressen ansässiger Praxen getätigt werden, womit nur ein Teil der Ärzteschaft der besagten Räume erreicht werden konnten. Die Ergebnisse dieser Studie sollen als richtungsweisend interpretiert werden und Ausgangspunkt für weitere Untersuchungen dienen. 


\section{Anhang}

\subsection{Anschreiben an die Praxen}

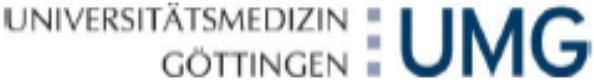

Univerelits:amedlyn Gotingen, von-Slebold-5r. 5, 37075 Gothoen

Adresse
Zentrum 16: Psychosoziale Medizin KIinik und Poliklinik für Psychiatrie und Psychotherapie Direktor: Prof. Dr. Jens Wiltfang

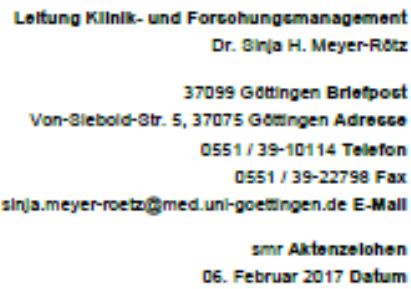

Unterstützung Promotion zur zahnmedizinischen Versorgung demenzkranker Patienten

Sehr geehrte Kolleginnen und Kollegen,

nach Abschluss meines Zahnmedizin-Studiums widme ich mich aktuell meiner Dissertation an der Universitätsmedizin Göttingen, Klinik für Psychiatrie und Psychotherapie, mit dem Titel „Deskriptive Studie zur zahnärztlichen Betreuung und Versorgung demenzkranker Patienten im Raum Göttingen“ und möchte Sie um lhre Unterstützung bitten.

Anhand einer deskriptiven Fragebogenstudie erhoffe ich mir Aufschluss darüber, wie viele demenzkranke Patienten in den zahnärztlichen Praxen im Großraum Göttingen behandelt werden. Des Weiteren sollen Ihre Erfahrungen mit der speziellen Betreuung dieser Patienten, sowie die individuelle Ausrichtung Ihrer Praxis und Ihres Teams auf demenzkranke Patienten erfasst werden.

Die Datenerfassung im Rahmen der Fragebögen erfolgt anonymisiert. Es werden keine personenidentifizierenden Merkmale erhoben, die einen Rückschluss auf eine bestimmte Person oder Praxis zulassen. Zudem können Sie den Fragebogen in einem vorfrankierten und adressierten Umschlag anonym zurücksenden. Durch die anonyme Rücksendung Ihres Fraqebogens willigen Sie qleichzeitiq in die Teilnahme an der Studie und die Auswertung und Verwendung der Daten im Rahmen der Dissertation ein.

Mit Ihrer Hilfe können wichtige Informationen zur zahnärztlichen Betreuung und Versorgung von demenzkranken Patienten gewonnen und Vorschläge zu einem individuellen Behandlungskonzept dieser Patientengruppe gemacht werden.

Bitte senden Sie den ausgefüllten Fragebogen bis zum 15. März 2017 mittels des frankierten Rückumschlags zurück.

Weiterführende Informationen finden Sie auf der Rūckseite dieses Anschreibens.

Ich bedanke mich schon im Voraus herzlich für Ihre Unterstützung!

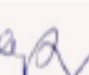

Anna Sophie Schaper

Universitatsmedizin Gottingen, Georq-August-Universitat Stiftung Offentlichen Rechts Vorstand Prof. Dr. Heyo K. Kroemer (forschung und Lehre, Sprecher des Vorstands) Dr. Martin Siess (Krankenversorgung) Dr. Sebastian Frextag (Wirtschaftstohrung und Administration) Sparkasse Gottingen (260 50001$)$ Kto: 448, IQAN: DE55 260500010000000448 , aiC: NOLADE21GOE 


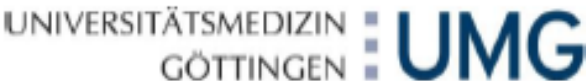

\section{Weiterführende Informationen für Studienteilnehmer}

Kontakt zur Studienleitung:

Klinik für Psychiatrie und Psychotherapie

Universitätsmedizin Göttingen

Dr. Sinja H. Meyer-Rötz

Von-Siebold-Str. 5

37075 Göttingen

E-Mail: sinja.meyer-roetz@med.uni-qoettingen.de

Telefon: 0551-39 10114

Doktorvater: Prof. Dr. med. Jens Wiltfang

Kurzfassung des Projekts:

Mit einer deskriptiven Studie im Großraum Göttingen soll herausgefunden werden wie Zahnarztpraxen und Zahnkliniken demenzerkrankte Patienten betreuen und behandeln und die zahnmedizinische Versorgung sicherstellen. Die Studie findet mittels Fragebögen mit Freitextanalyse und geschlossenen Fragen statt.

Die Datenerhebung soll zum einen Aufschluss über die Anzahl behandelter Patienten mit einer demenziellen Erkrankung geben und zum anderen sollen deren spezielle Betreuung und Besonderheiten in der zahnmedizinischen Behandlung, sowie Kenntnisse und Erfahrungen von Zahnärzten und Angestellten im Umgang mit demenzerkrankten Patienten erhoben werden.

Anhand der Ergebnisse sollen im Rahmen der Diskussion Rückschlüsse gezogen werden, wie die Versorgungsrealitāt von demenzerkrankten Patienten aussieht und welcher Kenntnisstand hinsichtlich des Umgangs mit demenzerkrankten Patienten bei Personal und Behandlern vorliegt. Mögliche Schulungsmaßnahmen und Lösungsansātze in Bezug auf die Thematik sollen im Rahmen der Dissertation recherchiert, zusammengetragen und analysiert werden.

\section{Datenschutz, rechtliche und ethische Aspekte}

Ihre Daten werden anonymisiert erhoben und es ist kein Rūckschluss auf Ihre Person oder Ihre Praxis möglich. Die Rücksendung der Fragebögen erfolgt anonym mittels eines frankierten und adressierten Rückumschlags. Die Daten werden in einer Datenbank unter Beachtung und Einhaltung der geltenden gesetzlichen Vorgaben gespeichert. Die Archivierung der Daten findet ebenfalls unter Beachtung und Einhaltung der geltenden gesetzlichen Vorgaben statt. Ein Rücktritt von der Einwilligung zur Studienteilnahme ist rūckwirkend aufgrund der anonymen Datenerhebung nicht möglich.

Die Studie wird unter Beachtung der Regularien der „good-clinical-practice" (GCP) durchgeführt und lag der Ethikkommission der Universitätsmedizin Göttingen, Von-Siebold-Str. 3, 37075 Göttingen zur Votierung vor. Die Votierung erfolgte positiv (Aktenzeichen: DOK_314_2015).

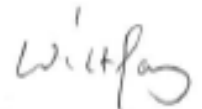

Prof. Dr. med. J. Wittang

Direktor der Klinik für Psychiatrie und

Psychotherapie der Universitätsmedizin Göttingen

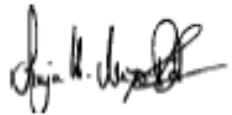

Dr. Sinja H. Meyer-Rötz

Studienleitung 


\subsection{Fragebogen}

\section{Fragebogen}

\section{Allgemeine Angaben zur Praxis und Zahnarzt/ärztin}

Alter des Zahnarztes/ärztin:

Wann erfolgte Ihr Staatsexamen?

$\square$ männlich $\square$ weiblich

$\square$ Gemeinschaftspraxis $\square$ Klinikum $\square$ alleinige Praxis $\quad \square$ sonstiges:

1. Wie konsultieren sie demenzkranke Patienten?

a) in Ihrer Praxis?

b) im Pflegeheim?

wenn Ja, $\quad \rightarrow \quad$ mit personeller Begleitung?

c) zu Hause?

wenn Ja, $\quad \rightarrow \quad$ ist dazu eine mobile Praxiseinrichtung vorhanden? $\square$ Ja $\square$ Nein

$\square$ Ja $\square$ Nein

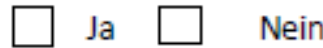

Ja $\square$ Nein

Ja $\square$ Nein

2. Ist Ihre Praxis für ältere Patienten gut erreichbar?

a) Hat Ihre Praxis Anschluss an öffentliche Verkehrsmittel?

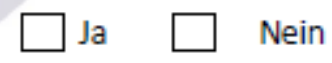

b) Gibt es ausgeschilderte Parkplätze in Praxisnähe?

Ja $\square$ Nein

c) Ist Ihre Praxis gut sichtbar ausgeschildert?

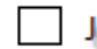

Ja $\square$ Nein

d) Besitzt Ihre Praxis einen barrierearmen Zugang?

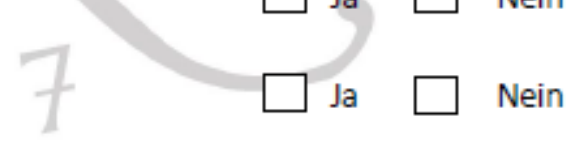

3. Ist Ihre Praxis besonders für demenzkranke Patienten ausgestattet?

a) Ist Ihre Praxis barrierearm?

Ja

Nein

b) Besitzt die Praxis behinderten gerechte Toiletten?

Ja $\square$ Nein

c) Benutzen Sie Hilfsmittel, wie z.B. ein Kissen zur Verbesserung der Lagerung?

Ja

Nein 
d) Verwenden Sie ein Zahnbänkchen (Mundsperre zum Offenhalten des Mundes) bei funktionell eingeschränkten Patienten?

Ja $\square$ Nein

e) Verwenden Sie weitere, nicht aufgelistete Hilfsmittel?

4) Welche Besonderheiten gibt es in der Weiterbildung und im Praxismanagement auf dem Gebiet der Gerodontologie?

a)haben Sie auf dem Gebiet der Gerodontologie eine besondere Weiterbildung? $\square$ Ja $\square$ Nein

b) Ist Ihr Personal auf dem Gebiet der Gerodontologie besonders weitergebildet? $\square$ Ja $\square$ Nein

c)Verwenden Sie im Patientengespräch spezielle Anschauungsmaterialien, wie z.B.

Flyer oder Modelle?

Ja $\square$ Nein

d) Findet das Patientengespräch mit demenzkranken Patienten über Dritte (Pflegepersonal, Angehörige) statt?

Ja $\square$ Nein

e) Können Sie bei Terminen mit demenzkranken Patienten mehr Zeit einplanen?

Ja $\square$ Nein

f) Legen Sie für demenzkranke Patienten im Vorfeld eine bestimmte Recall-Struktur fest?

Ja $\square$ Nein

g) Gibt es Besonderheiten in der Gesprächsführung mit demenzkranken Patienten?

5) Welche Besonderheiten gibt es in der zahnmedizinischen Versorgung von demenzkranken Patienten?

a) Stellen Sie bei motorisch eingeschränkten Patienten die Indikation zur Zahnextraktion strenger?

$\square$ Ja $\square$ Nein

b) Stellen Sie den Patienten früher von festsitzenden auf herausnehmbaren Zahnersatz um?

$\square$ Ja $\square$ Nein

c)Empfehlen Sie demenzkranken Patienten eine bestimmte Putztechnik? $\quad \square$ Ja $\quad \square$ Nein

Wenn ja, welche? 
d) Empfehlen Sie demenzkranken Patienten spezielle Mundhygienemittel?

$\square$ Ja Nein Wenn ja,

A) Empfehlen Sie motorisch eingeschränkten Patienten eine spezielle Zahnbürste?

Elektrische Zahnbürste

Superbrush

(3-flächig reinigend)
Handzahnbürste mit

angepassten Griff

Sonstige:

B) Welche Hilfsmittel für die Interdentalraumpflege empfehlen Sie motorisch eingeschränkten Patienten?

$\square$ Zahnseide $\square$ Interdentalraumbürsten $\square$ Dreikanthölzer

$\square$ Sonstige:

C) Empfehlen Sie Mundspüllösungen?

Nein

D) Welche Hilfsmittel zur Prothesenreinigung empfehlen Sie?

Ultraschallbad

Zahnbürste und Zahnpasta

Reinigungstabletten

Sonstige:

e) Behandeln Sie demenzkrankte Patienten mit niedriger Compliance mit sedierenden

Medikamenten?

Ja

Nein

Wenn ja, mit welchen Medikamenten?

f) Behandeln Sie demenzkranke Patienten unter Vollnarkose?

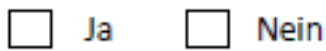

g) Überweisen Sie demenzkranke Patienten für eine stationäre Behandlung unter Vollnarkose?

h) Stehen Sie in Kontakt mit Hausärzten oder anderen Ärzten bei der Behandlung demenzkranker Patienten?

$\square$ Nie $\square$ Selten $\square$ Häufig $\square$ Immer

i) Legen Sie Wert auf eine funktionell möglichst einfache Prothesengestaltung? $\square$ Ja $\square$ Nein 
j) Gibt es weitere Besonderheiten in der zahnmedizinischen Versorgung demenzkranker Patienten in Ihrer Praxis?

6) Gibt es intraorale Besonderheiten bei demenzkranken Patienten?

a) Stellen Sie bei demenzkranken Patienten eine Unterversorgung in Bezug auf die nicht betroffene Altersgruppe fest?

$\square$ trifft nicht zu $\square$ trifft eher nicht zu $\square$ unentschlossen $\square$ trifft eher zu $\quad \square$ trifft voll zu

b) Stellen Sie bei demenzkranken Patienten eine schlechtere Mundhygiene als bei der nicht betroffenen Altersgruppe fest?

$\square$ trifft nicht zu $\square$ trifft eher nicht zu $\square$ unentschlossen $\square$ trifft eher zu $\quad \square$ trifft voll zu

c) Stellen Sie bei demenzkranken Patienten häufiger intraorale Auffälligkeiten und Erkrankungen fest?

Ja $\square$ Nein

Wenn Ja, $\quad$ trifft nicht zu trifft eher unentschlossen trifft eher zu trifft voll zu nicht zu

A) Karies

B) Gingivitis

C) Druckstellen

D) Parodontitis

E) Prothesenstomatitis

F) Xerostomie

G) Hypersalivation

H) Mundschleimhautentzündungen

I) kanzerogene

Veränderungen $\square$ 
d) Ist ein demenzkranker Patient Ihrer Meinung nach in der Lage eine eigenständige adäquate Mundhygiene auszuführen?

$\square$ trifft nicht zu $\square$ trifft eher nicht zu $\square$ unentschlossen $\square$ trifft eher zu $\quad \square$ trifft voll zu

7) Unterliegen demenzkranke Patienten in Ihrer Praxis einer besonderen Beobachtung? Wenn ja,

a) Verwenden Sie spezielle Screening-Bögen oder spezielle Tests zur Erfassung demenzkranker Patienten?

$\square$ Ja $\square$ Nein

Wenn ja, welche?

b) Kommen demenzkranke Patienten in Begleitung Dritter in Ihre Praxis?

$\square \quad$ Nie $\square$ Selten $\square$ Häufig $\square$ Immer

c) Gibt es weitere Besonderheiten in der Beobachtung demenzkranker Patienten in Ihrer Praxis?

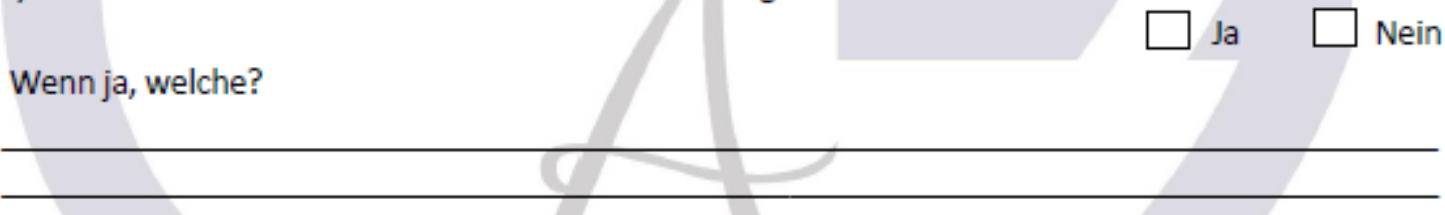

8) Wo sehen Sie Verbesserungsbedarf im Umgang und der zahnmedizinischen Versorgung demenzkranker Patienten?

9) Wie viele demenzkranke Patienten werden in Ihrer Praxis schätzungsweise in einem Quartal behandelt?

Patienten

a) Wie viel Prozent sind davon über 65 Jahre?

Prozent

10) Hätten Sie Interesse an einer Fortbildung für den Umgang mit demenzkranken Patienten?

Ja $\square$ Nein 


\section{Literaturverzeichnis}

Arnetzl GV, Arnetzl G (2013): Zahnpflege im Alter. Stomatologie $110(4 / 5), 12-16$

Bär C, Reiber T, Nitschke I (2009): Zahnmedizinische Probleme bei alten Patienten. Zahnarztl Mitt $\underline{99}$ (24), 44-49

Barth S (1998): Die schriftliche Befragung. Online verfügbar unter: https://www.hb.fhmuenster.de/opus/fhms/volltexte/2011/713/pdf/SchriftlicheBefragung.pdf. Zuletzt geprüft am 19.07.2018

Baumgartner W, Schimmel M, Müller F (2015): Zur Mundegesundheit und zahnärztlichen Betreuung pflegebedürftiger Betagter. Swiss Dent J $\underline{125}$ (4), 459-469

Besimo CE (2009): Medizinisch-psychosoziales Screening des alternden Menschen in der zahnärztlichen Praxis. Schweiz Monatsschr Zahnmed 119 (12), 1237-1243

Besimo CE, Besimo-Meyer RH (2015): Orale Gesundheit von Menschen mit Demenz. Schweiz Z Ganzheitsmed 27 (1), 44-49

Besimo CE, Besimo-Meyer RH (2016): Demenz in der oralen Medizin: Erkennen von Zeichen der Erkrankung - Einschätzen von Ressourcen. Schweiz Z Ganzheitsmed 28 (1), 45-50

Bickel H: Epidemiologie und Gesundheitsökonomie. In: Wallesch CW, Förstl H (Hrsg.): Demenzen. 2. Auflage; Thieme, Stuttgart 2012, 18-35

Bornemann-Cimenti H, Wejbora M, Michaeli K, Kern-Pirsch C, Sandner-Kiesling A (2012): Schmerzerfassung bei Demenz. Nervenarzt $\underline{83}$ (4), 458-466

Bühler S (2014): Mini-Mental Status-Test (MMST) - Der schnelle Standardtest. ergopraxis 7 (2), 34-35

DAK: Pflege-Report 2015. So pflegt Deutschland. DAK Gesundheit, Hamburg 2015. Online verfügbar unter: https://www.dak.de/dak/download/pflegereport-2015-1701160.pdf. Zuletzt geprüft am 30.06.2018

Dettbarn-Reggentin MA, Reggentin H: Abschlussbericht: Mundgesundheit und Lebensqualität mobilitätsbeeinträchtiger betreuungsbedürftiger Menschen in häuslicher und stationärer Versorgung. Konzept der aufsuchenden ambulanten zahnärztlichen Versorgung - "ZahnRad". ISGOS-Berlin, Berlin 2016. Online verfügbar unter: https://www.isgos.de/cms/images/stories/PDFs2017/ZahnRAD-Abschluss-2016-2.pdf. Zuletzt geprüft am 04.07.2018

Diekmann A: Empirische Sozialforschung: Grundlagen, Methoden, Anwendungen. Rowohlt, Reinbek bei Hamburg 1995

Drach LM: Demenz mit Lewy-Körperchen und Parkinson-Demenz - Gemeinsamkeiten und Unterschiede. In: Mahlberg R, Gutzmann H (Hrsg.): Demenzerkrankungen: erkennen, behandeln und versorgen. 1. Auflage; Deutscher Ärzte-Verlag, Köln 2009, 31-38

Gasser T, Maetzler W: Molekulargenetik und Neurobiologie neurodegenerativer Demenzen. In: Wallesch CW, Förstl H (Hrsg.): Demenzen. 2. Auflage; Thieme, Stuttgart 2012, 36-51

Glenz F, Brand C, Besimo C, Marinello C (2015): Der ältere Patient: Kein Grund zur Sorge!? Die Notwendigkeit eines Gesundheits-Screenings von älteren und betagten Patienten im Praxisalltag. Swiss Dent J $\underline{125}$ (4), 469-474

Grossmann J, Pirker W (2004): Klinik und Therapie der Parkinson-Psychose. J Neurol Neurochir Psychiatr $\underline{5}(2), 42-48$ 
Gumpp V, Henß H: Tumoren der Lippen und Mundhöhle (ohne Speicheldrüse). Comprehensive Cancer Center Freiburg, Freiburg 2014. Online verfügbar unter: https://www.uniklinikfreiburg.de/fileadmin/mediapool/09_zentren/cccf/pdf/cccf_kkr_kodierhilfe_lippen_mundhoehle_tu mor.pdf. Zuletzt geprüft am 30.06.2018

Hallauer J, Bienstein C, Lehr U, Rönsch H: SÄVIP-Studie zur ärztlichen Versorgung in Pflegeheimen. Vincentz Network Marketing Service, Hannover 2005. Online verfügbar unter: https://docplayer.org/7768209-Saevip-studie-zur-aerztlichen-versorgung-in-pflegeheimen.html.

Zuletzt geprüft am 25.01.2019

Hamann GF: Vaskuläre Demenzen. In: Wallesch CW, Förstl H (Hrsg.): Demenzen. 2. Auflage; Thieme, Stuttgart 2012, 272-282

Jäger S, Köster-Schmidt A, Schade M, Heudorf U (2009): Mundhygiene und Mundgesundheit bei Bewohnern von Altenpflegeheimen. Bundesgesundheitsblatt Gesundheitsforschung Gesundheitsschutz 52 (10), 927-935

Jordan AR, Micheelis W: Fünfte Deutsche Mundgesundheitsstudie-(DMS V). Institut der deutschen Zahnärzte. Deutscher Zahnärzteverlag, Köln 2016. Online verfügbar unter: https://www.bzaek.de/fileadmin/PDFs/dms/Statement_DrJordan.pdf. Zuletzt geprüft am 30.06.2018

Kessler J, Calabrese P, Kalbe E, Berger F (2000): DemTect: Ein neues Screening-Verfahren zur Unterstützung der Demenzdiagnostik. Psycho 26 (6), 343-347

Kluding I (2013): Zahnpflege im Alter. proDente e.V. Online verfügbar unter: https://www.prodente.de/prophylaxe/einzelansicht/haeusliche-zahnpflege/zahnpflege-im-alter.html. Zuletzt geprüft am 30.06.2018

Knabe C, Kram P (1997): Dental care for institutionalized geriatric patients in Germany. J Oral Rehabil 24 (12), 909-912

Kratz CD, Schleppers A, Iber T, Geldner G (2005): Pharmakologische Besonderheiten und Probleme des älteren Patienten. Anaesthesist 54 (5), 467-475

Kretsch M: Zahnärztliche Hausbesuche bei Patienten $\geq 62$ Jahren im häuslichen Setting unter besonderer Berücksichtigung internistischer Erkrankungen und wirtschaftlicher Aspekte. Med. Diss. München 2016

Krömer T (2018): Verbesserung der Mundhygiene pflegebedürftiger Menschen durch Schulungen des Pflegepersonals im Kreis Ostholstein. Ergebnisse einer Interventionsstudie. Gesundheitswesen $\underline{80}(4), 389$

Kuhlmey A, Tesch-Römer C: Autonomie trotz Multimorbidität im Alter: Eine Einführung. In: Kuhlmey A, Tesch-Römer C (Hrsg.): Autonomie trotz Multimorbidität. Ressourcen für Selbstständigkeit und Selbstbestimmung im Alter. 1. Auflage; Hogrefe, Göttingen 2013, 9-21

Liakoni E, Liechti ME (2015): Lachgas in der Zahnmedizin. Rettung oder Verharmlosung? Swiss Dent J 125 (10), 1099-1104

Ludwig E: Zahn-, Mund- und Zahnersatzpflege in der Altenpflegeausbildung. Landeszahnärztekammer Baden-Württemberg, 2009. Online verfügbar unter: https://lzkbw.de/fileadmin/user_upload/4.Presse/60.Hintergrundinformationen/10.Publikationen/WrigleyLudwig-2012.pdf. Zuletzt geprüft am 05.08.2018

Ludwig E: Mobile Behandlungseinheiten in der Behandlung pflegebedürftiger Menschen. Landeszahnärztekammer Baden-Württemberg, 2010. Online verfügbar unter: https://lzkbw.de/fileadmin/user_upload/1.Zahn\%C3\%A4rzte/110.Alters-

_und_Behindertenzahnheilkunde/40.Barrierefreiheit/Mobile-Behandlungseinheiten.pdf. Zuletzt geprüft am 30.06.2018 
Ludwig E (2016): Alterszahnheilkunde - ein erfolgreiches Praxiskonzept. Wissen Kompakt 10 (4), $159-172$

Ludwig E, Bublitz R, Elsäßer G, Richtmann C (2014): Sektorenübergreifender Behandlungspfad zur Gebisssanierung. Freie Zahnarzt $\underline{58}$ (7/8), 78-86

Mahlberg R, Gutzmann H: Einteilung: Krankheitsbilder. In: Mahlberg R, Gutzmann H (Hrsg.): Demenzerkrankungen: erkennen, behandeln und versorgen. 1. Auflage; Deutscher Ärzte-Verlag, Köln 2009, 3-10

Maier H, Dietz A, Zielinski D, Jünemann KH, Heller WD (1990): Risikofaktoren bei Plattenepithelkarzinomen der Mundhöhle, des Oropharynx, des Hypopharynx und des Larynx. Dtsch Med Wochenschr 115, 843-850

Maier W, Barnikol UB (2014): Neurokognitive Störungen im DMS-5. Nervenarzt 85 (5), 564-570

Marmy M, Matt F (2003): Kenntnisse in der Mund- und Zahnhygiene des Pflegepersonals eines psychogeriatrischen Heims. Schweiz Monatsschr Zahnmed 113 (7), 794-800

Mathers F: Gerodontologie: Was Demenzpatienten brauchen. DZW. Zahnärztlicher Fachverlag, Herne 2015. Online verfügbar unter: https://www.dzw.de/gerodontologie-was-demenzpatientenbrauchen. Zuletzt geprüft am 30.06.2018

Mielke R, Kessler J: Alzheimersche Erkrankung und andere Demenzen. Hogrefe, Göttingen 1994

Nitschke I, Kaschke I (2011): Zahnmedizinische Betreuung von Pflegebedürftigen und Menschen mit Behinderungen. Bundesgesundheitsblatt Gesundheitsforschung Gesundheitsschutz 54 (9/10), 1073-1082

Nitschke I, Ilgner A, Reiber T (2004): Zur Etablierung der Seniorenzahnmedizin in der zahnärztlichen Ausbildung. Dtsch Zahnarztl Z 59 (3), 163-167

Nitschke I, Stillhart A, Kunze J (2015): Zur Inanspruchnahme zahnmedizinischer Dienstleistungen im Alter. Swiss Dent J 125 (4), 475-490

Nuri M (2015): Gemeinsame Kostenlast trägt sich leichter. Freie Zahnarzt $\underline{59}$ (5), 46-47

Rapp MA: Alzheimer-Demenz. In: Mahlberg R, Gutzmann H (Hrsg.): Demenzerkrankungen: erkennen, behandeln und versorgen. 1. Auflage; Deutscher Ärzte-Verlag, Köln 2009, 11-18

Reißmann DR, Lamprecht R: Zahn- und Mundgesundheit im Alter. Walter de Gruyter, Berlin 2017

Rundshagen I (2014): Postoperative kognitive Dysfunktion. Dtsch Arztebl 111 (8), 119-125

Schlegel J, Neff F: Neuropathologie. In: Wallesch CW, Förstl H (Hrsg.): Demenzen. 2. Auflage; Thieme, Stuttgart 2012, 67-86

Schmitt S (2017): Zahngesund im Pflegeheim. Freie Zahnarzt 61 (12), 14-16

Schulte-Herbrüggen O, Hellweg R: Vaskuläre Demenzen. In: Mahlberg R, Gutzmann H (Hrsg.): Demenzerkrankungen: erkennen, behandeln und versorgen. 1. Auflage; Deutscher Ärzte-Verlag, Köln 2009, 19-31

SGZBB (Schweizerische Gesellschaft für die zahnmedizinische Betreuung Behinderter und Betagter) (2015): Gerodontologie. Mundgesundheitsmodell der SGZBB für die zahnmedizinische Betreuung von pflegebedürftigen Betagten. Swiss Dent J $\underline{125}$ (4), 457-458

Sinner B: Neurologische Erkrankungen. In: Graf BM, Sinner B, Zink W (Hrsg.): Anästhesie bei alten Menschen. 1. Auflage; Thieme, Stuttgart 2009, 188-198

Tölle R, Windgassen K: Psychiatrie. 17. Auflage; Springer Medizin, Berlin 2014 
Wallesch CW, Förstl H: Demenz mit Lewy-Körperchen. In: Wallesch CW, Förstl H (Hrsg.): Demenzen. 2. Auflage; Thieme, Stuttgart 2012 , 228-232

Wernke A, Steinbock S: Menschen mit Demenz in der Arztpraxis. Demenz-Servicezentrum Regio Aachen/Eifel. Verbraucherzentrale Nordrhein-Westfalen, Düsselsorf 2015. Online verfügbar unter: https://www.demenz-service-aachen-

eifel.de/tl_files/aachen_eifel/2015/50_Broschuere_Demenz_Arztpraxis_Aachen_Eifel.pdf. Zuletzt geprüft am 30.06.2018

Wöstmann B (2003): Zahnersatz und Gesundheit bei Senioren. Zahnarztl Mitt 93 (9), 44-48

Zenthöfer A, Hassel AJ, Rammelsberg P (2016): Alterszahnheilkunde. Mundgesundheit und Lebensqualität beim alternden Patienten. Wissen Kompakt $\underline{10}$ (4), 145-158 